\section{Evaluating the stress-response of dental students to the dental 2 school environment.}

3 Katarzyna Mocny-Pachońska DDS, $\mathrm{PhD}^{1}$, Rafał Doniec $\mathrm{PhD}^{2}$, Agata Trzcionka DDS, $\mathrm{PhD}^{1}$, Marek

4 Pachoński DDS $^{3}$, Natalia Piaseczna ${ }^{2}$, Szymon Sieciński ${ }^{2}$, Aleksandra Osadcha ${ }^{4}$ Patrycja Łanowy $^{1}$,

5 Marta Tanasiewicz DDS, Prof. ${ }^{1}$

$6 \quad{ }^{1}$ Medical University of Silesia, Faculty of Medical Science

7 Department of Conservative Dentistry with Endodontics

$8 \quad$ Pl. Akademicki 17, 41-902 Bytom, Poland

$9 \quad 2$ Silesian University of Technology, Faculty of Biomedical Engineering

10 Department of Biosensors and Biomedical Signal Processing

11 Roosevelta 40, 41-800 Zabrze, Poland

$12{ }^{3}$ Pachońscy Dental Clinic

13 Lubliniecka 38, 42-288 Strzebiń, Poland

$14{ }_{4}^{4}$ Silesian University of Technology, Institute of Mathematics

15 Kaszubska 23, 44-100 Gliwice, Poland

16 Corresponding Author:

17 Katarzyna Mocny-Pachońska DDS, PhD

18 Department of Conservative Dentistry with Endodontics

19 Pl. Akademicki 17, 41-902 Bytom, Poland

20 Email address: kpachonska@sum.edu.pl

21

22

23

24

25

26

27

\begin{tabular}{|c|c|}
\hline Formatted & $\ldots[1]$ \\
\hline Style Definition & $\ldots[18]$ \\
\hline Style Definition & $\ldots[17]$ \\
\hline Style Definition & $\ldots[16]$ \\
\hline Style Definition & $\ldots[15]$ \\
\hline Style Definition & $\ldots[14]$ \\
\hline Style Definition & $\ldots[13]$ \\
\hline Style Definition & $\ldots[12]$ \\
\hline Style Definition & $\ldots[11]$ \\
\hline Style Definition & $\ldots[10]$ \\
\hline Style Definition & $\ldots[9]$ \\
\hline Style Definition & $\ldots[8]$ \\
\hline Style Definition & $\ldots[7]$ \\
\hline Style Definition & $\ldots[6]$ \\
\hline Style Definition & $\ldots[5]$ \\
\hline Style Definition & $\ldots[4]$ \\
\hline Style Definition & $\ldots[3]$ \\
\hline Deleted: Impartial M & Measurement of Dental Studentș'[19] \\
\hline Formatted & $\ldots[20]$ \\
\hline Formatted & $\ldots[21]$ \\
\hline Formatted & $\ldots[22]$ \\
\hline Formatted & $\ldots[23]$ \\
\hline Formatted & $\ldots[24]$ \\
\hline Deleted: School of Me & Tedicine with the Division of Dentistry \\
\hline Formatted & $\ldots[25]$ \\
\hline Formatted & $\ldots[26]$ \\
\hline Formatted & $\ldots[27]$ \\
\hline Formatted & $\ldots[28]$ \\
\hline Deleted: Roosvelta & \\
\hline Formatted & $\ldots[29]$ \\
\hline Formatted & $\ldots[30]$ \\
\hline Formatted & $\ldots[31]$ \\
\hline Formatted & $\ldots[32]$ \\
\hline Formatted & $\ldots[33]$ \\
\hline Formatted & $\ldots[34]$ \\
\hline Formatted & $\ldots[35]$ \\
\hline Deleted: MD & \\
\hline Formatted & $\ldots[36]$ \\
\hline Formatted & $\ldots[37]$ \\
\hline Formatted & $\ldots[38]$ \\
\hline Formatted & $\ldots[39]$ \\
\hline Field Code Changed & $\ldots[40]$ \\
\hline Formatted & $\ldots[41]$ \\
\hline Formatted & $\ldots[42]$ \\
\hline Formatted & $\ldots[43]$ \\
\hline Formatted & $\ldots[2]$ \\
\hline
\end{tabular}




\section{Abstract}

40 Introduction and Objective. Dentists experience high amounts of professional stress beginning 41 with their student years in dental school. This stress, given jts early onset, may negatively impact 42 the personal and professional lives, of these individuals, as well as the quality of their clinical work. 43 We sought to create an objective scale to evaluate the levels of stress in students at different stages of their education as well as in practicing physicians.

Materials and Methods. 30 dental students participated in this study with 10 students each selected from junior, mid-senior, and senior classes. They were randomly divided jnto two groups in which one group was subjected to stressors while the other group was not JINS MEME ES R (JINS) smart glasses and Garmin Vivoactive 3 smartwatches, were used to obtain data including electrooculography (EOG), heart rate (HR), and accelerometer (ACC) and gyroscope (GYRO) feedback, while the subjects performed a dental exercise on a phantom tooth.

Results. The heart rates of more experienced students were lower than those of the junior students The EOG, ACC, and GYRO signals showed multiple differences in the measurement of amplitudes and frequency of episodes.

Conclusion. Our pilot results show that electronic tools like smart glasses with software and sensors, are useful for monitoring the stress levels of dental students in preclinical operating conditions. We would like to further assess the stress levels in students performing dental procedures on phantom teeth and in later clinical jnteractions with patients.

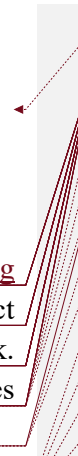

Formatted

Deleted: ...nd ...bjective. ...entists ...xperience ...igh46]

Formatted

Deleted: further stages of the project covering groups of

Formatted

Deleted: and

Formatted

Deleted:

Deleted:

Formatted

Deleted:

Deleted: :

Formatted

Deleted: There were

Formatted

Formatted

Deleted: participants

Formatted

Formatted

Formatted

Deleted: 10

Deleted:

Formatted

Deleted:

Formatted

Deleted: 10

Formatted

Deleted: students of a dentistry course, who

Formatted

Deleted:

Formatted

Deleted:

Formatted

Formatted

Deleted: -

Formatted

Deleted: and

Formatted

Deleted:

Formatted

Deleted: subjected to stress factors.

Formatted

Deleted:

Formatted

(eye-sensing device for researchers

Formatted

Deleted:

Formatted

Deleted:

Formatted

Deleted: smartwatch

Formatted

Deleted:

Formatted

Deleted:

Formatted

Deleted:

Formatted

Deleted: 
$\underline{\text { their student years working with phantom teeth through their preclinical and clinical practice years }}$ [1].

Stress related to dental school can negatively, impact a dentist's personal and professional life particularly with regard to the quality of their clinical work [2-6]. Stress resulting from external physical or mental factors can affect an individual's physical and psychological well-being and may reduce work efficiency, and impact the accuracy of diagnostic and therapeutic decisions $[2,4]_{x}$.

It is difficult to predict and control reactions to external stressogenic situations. During stressful conditions, the hypothalamus-pituitary-adrenal (HPA) axis and the sympathetic nervous system (SNS) are activated. The nervous system becomes aroused in periods of stress or danger, which affects an individual's reactions. Responses to stressful situations vary among individuals due to diverse perceptions of circumstances. The stress response is more highly aroused when a stressor is directed in a personal manner and continues with the ongoing risk of threat $[7,8]$.

Studies have shown that students from medical universities are more prone to psychological problems due to unique stressors, including the demands of their education and special expectations compared to students of humanities and technical faculties [3]. Dyrye et al. (2006) reported that medical students experience high levels of anxiety and depression [9]. Mohannad et al. (2019) and Brazeau et al. (2014) confirmed that depression in dental students and interns appears more frequently than in the general population, with evidence of mental health deterioration over the course of their medical training [10,11]. Faris et al. (2016) emphasized that the third and fifth years of dental and medical studies are highly, associated with depressive symptoms in students [1].

Dental school is a significant source of stress for dental students who are required to practice in a professional manual workshop for clinical work, establish direct patient contact, and manage patient stress. These stressors are in addition to their rigorous, educational challenges, which include providing care for patients and performing treatments that could cause harm if performed jimproperly.

Dental students must deal with life-threatening conditions, work long hours, undertake a demanding workload, and master an intense theoretical education [4]. Studies show that young dental students can significantly improve complicated fine, motor skills, such as performing dental work while looking in a mirror, with practice [12].

Dental students appear to be more at-risk for stress than students from other faculties, including medical students, because the need to acquire extensive knowledge is combined with manual training, which is practiced in a preclinical simulation center and then in clinical practice [13].

When training in clinical practice, students have direct contact with patients who may be in pain and fearful of dental examinations and procedures [13]. Our findings support the results demonstrated by Abu-Ghazaleh et al.(2016), that stress in dental students at the University of Jordan increased over the years of the clinical training; fifth-year students showed especially high levels

\begin{tabular}{|c|c|}
\hline Formatted & $\ldots[127]$ \\
\hline \multicolumn{2}{|c|}{ Deleted: and then with patients during the } \\
\hline Formatted & $\ldots[129]$ \\
\hline Formatted & $\ldots[130]$ \\
\hline \multicolumn{2}{|c|}{ Deleted: next during } \\
\hline Formatted & $\ldots[131]$ \\
\hline \multicolumn{2}{|l|}{ Deleted: } \\
\hline \multicolumn{2}{|c|}{ Deleted: from the } \\
\hline \multicolumn{2}{|l|}{ Deleted: } \\
\hline \multicolumn{2}{|l|}{ Deleted: } \\
\hline Formatted & $\ldots[132]$ \\
\hline Formatted & $\ldots[133]$ \\
\hline Formatted & $\ldots[134]$ \\
\hline \multicolumn{2}{|c|}{ Deleted: environment can have a negative } \\
\hline \multicolumn{2}{|c|}{ Deleted: on their } \\
\hline \multicolumn{2}{|l|}{ Deleted: } \\
\hline Formatted & $\ldots[135]$ \\
\hline Formatted & $\ldots[136]$ \\
\hline Formatted & $\ldots[137]$ \\
\hline Formatted & $\ldots[138]$ \\
\hline \multicolumn{2}{|c|}{ Deleted: lives - } \\
\hline \multicolumn{2}{|c|}{ Deleted: } \\
\hline Formatted & $\ldots[143]$ \\
\hline Formatted & $\ldots[145]$ \\
\hline \multicolumn{2}{|l|}{ Deleted: } \\
\hline Formatted & $\ldots[146]$ \\
\hline \multicolumn{2}{|c|}{ Deleted: subsequent } \\
\hline Formatted & $\ldots[144]$ \\
\hline \multicolumn{2}{|l|}{ Deleted: } \\
\hline Formatted & $\ldots[148]$ \\
\hline \multicolumn{2}{|l|}{ Deleted: } \\
\hline Formatted & $\ldots[139]$ \\
\hline \multicolumn{2}{|l|}{ Deleted: } \\
\hline Formatted & $\ldots[140]$ \\
\hline \multicolumn{2}{|l|}{ Deleted: } \\
\hline Formatted & $\ldots[141]$ \\
\hline \multicolumn{2}{|l|}{ Deleted: } \\
\hline Formatted & $\ldots[142]$ \\
\hline \multicolumn{2}{|l|}{ Deleted: } \\
\hline Deleted: & \\
\hline Formatted & $\ldots[147]$ \\
\hline Formatted & $\ldots[149]$ \\
\hline Deleted: & \\
\hline Deleted: as & \\
\hline Formatted & $\ldots[150]$ \\
\hline Formatted & $\ldots[151]$ \\
\hline Deleted: & \\
\hline Formatted & $\ldots[152]$ \\
\hline Deleted: & \\
\hline Formatted & $\ldots[153]$ \\
\hline Deleted: & \\
\hline Formatted & $\ldots[154]$ \\
\hline Deleted: & \\
\hline Formatted & $\ldots[155]$ \\
\hline Deleted: tha & \\
\hline Formatted & $\ldots[156]$ \\
\hline Deleted: & \\
\hline Formatted & $\ldots[157]$ \\
\hline Deleted: & \\
\hline
\end{tabular}


of psychological stress due to an increased awareness of the expectations and responsibilities of their work [14]. The negative impact of depression on dental students is replaced by, stress resulting from the need to cope with professional duties and full-time clinical work, and the need to simultaneously carry out continuing postgraduate education [15].

Stress adversely affects the quality of patient care, patient safety, and professionalism and its effects extend to the community at large [1]. Therefore, given the high rate of depressive symptoms in dental students, stress levels and their causative factors should be determined and preventive measures should be considered [16].

Davidovich et al.'s (2015), research comparing students and experienced professionals shows that stress levels are significantly higher among newcomers to clinical practice, Junior, students rely on theoretical knowledge from preclinical classes, which may not yet be sufficient, and are typically anxious about using dental equipment with precision [16].

Piazza-Waggoner et al. and Davidovich et al.'s (2003, 2015) investigations confirmed that dental students practicing on patients in a clinical setting have a particularly high level of stress during the implementation of procedures related to invasive work on patients, especially when practicing on a child $[3,16]$.

The environment in which students perform their clinical work js also significant. The possibility of peing able to perform tasks in silence, under time constraints while being able to complete the task satisfactorily, and the knowledge of how to deal with stressful situations are factors that influence a student's quality of work [17, 18]. .

Several recent studies have investigated the effectiveness of interventions such as psychoeducational interventions, music therapy, and Progressive Muscle Relaxation (PMR) to cope with student anxiety or stress before academic exams or evaluations [19, 20,21].

Researchers have, not yet confirmed that relaxation or stress management techniques are effective in reducing the fear of working in a clinical setting [16]. It is difficult to accurately identify a person's cognitive state but it is important to have an objective real time evaluation for dentists because their cognitive state can influence their performance.

Researchers typically rely on psychological measurements to collect data [22, 23]. However future research should determine whether differences in the prevalence of severe depressive symptoms among students in different disciplines are due to the innate nature of the discipline, the type of curriculum being studied, or the intellectual, and manual skills required, [1].

\section{Objectives}

We conducted an arbitrary, measurable study of the arousal level of 30 dental students in their third, fourth, and fifth years of study. Results were measured by blink occurrence, speed, and strength; vertical and horizontal eye movement; head movement (rotation roll, pitch, and yaw), and changes in heart rate measured by JINS smart glasses and a GPS smartwatch, with and without

\begin{tabular}{|c|c|}
\hline Formatted & $\ldots[253]$ \\
\hline \multicolumn{2}{|l|}{ Deleted: [12 } \\
\hline Formatted & $\ldots[255]$ \\
\hline Formatted & $\ldots[256]$ \\
\hline \multicolumn{2}{|c|}{ Deleted: after graduation by the } \\
\hline Formatted & $\ldots[257]$ \\
\hline \multicolumn{2}{|l|}{ Deleted: } \\
\hline \multicolumn{2}{|l|}{ Deleted: 13} \\
\hline Formatted & $\ldots[258]$ \\
\hline Formatted & $\ldots[259]$ \\
\hline \multicolumn{2}{|l|}{ Deleted: It } \\
\hline Formatted & $\ldots[260]$ \\
\hline \multicolumn{2}{|c|}{ Deleted: . In other words, } \\
\hline \multicolumn{2}{|c|}{ Deleted: effect extends } \\
\hline Formatted & $\ldots[261]$ \\
\hline Formatted & $\ldots[262]$ \\
\hline \multicolumn{2}{|c|}{ Deleted: for dental students } \\
\hline Formatted & $\ldots[263]$ \\
\hline \multicolumn{2}{|l|}{ Deleted: 13} \\
\hline Formatted & $\ldots[264]$ \\
\hline Formatted & $\ldots[265]$ \\
\hline \multicolumn{2}{|l|}{ Deleted: } \\
\hline Formatted & $\ldots[266]$ \\
\hline \multicolumn{2}{|l|}{ Deleted: } \\
\hline Formatted & $\ldots[267]$ \\
\hline \multicolumn{2}{|c|}{ Deleted: on the comparison of the results of } \\
\hline Formatted & $\ldots[268]$ \\
\hline \multicolumn{2}{|l|}{ Deleted: } \\
\hline \multicolumn{2}{|l|}{ Deleted: } \\
\hline Formatted & $\ldots[270]$ \\
\hline \multicolumn{2}{|l|}{ Deleted: } \\
\hline Formatted & $\ldots[269]$ \\
\hline Formatted & $\ldots[271]$ \\
\hline \multicolumn{2}{|l|}{ Deleted: } \\
\hline Formatted & $\ldots[272]$ \\
\hline \multicolumn{2}{|l|}{ Deleted: } \\
\hline Formatted & $\ldots[273]$ \\
\hline \multicolumn{2}{|l|}{ Deleted: } \\
\hline Formatted & $\ldots[274]$ \\
\hline \multicolumn{2}{|c|}{ Deleted: , as junior } \\
\hline Formatted & $\ldots[275]$ \\
\hline Deleted: & \\
\hline Formatted & $\ldots[276]$ \\
\hline Deleted: & \\
\hline Formatted & $\ldots[277]$ \\
\hline Deleted: & \\
\hline Formatted & $\ldots[278]$ \\
\hline Deleted: gai & \\
\hline Deleted: & \\
\hline Formatted & $\ldots[279]$ \\
\hline Deleted: & \\
\hline Formatted & $\ldots[280]$ \\
\hline Formatted & [281] \\
\hline Deleted: & \\
\hline Deleted: wh & \\
\hline Formatted & $\ldots[282]$ \\
\hline Deleted: afre & \\
\hline Formatted &..$[283]$ \\
\hline Deleted: & \\
\hline
\end{tabular}


stress stimulation. We created our own protocol for stress simulation based on literature data [24. 25]. The first measured stressor was shaping a cavity in a tooth in a limited time under the careful supervision of a teacher. The second stressor was creating the shape of a cavity similar to the matrix of the cavity. The third stressor was a lack of information on how to prepare the cavity. The control group of students without the stress simulation protocol underwent a session of muscle relaxation, music therapy, and were given full information about the task to be performed. We sought to standardize, the measurement method that could be used in further studies using JINS smart glasses.

\section{Materials and Methods}

\section{Technology used}

We used JINS MEME ES R (JINS) smart glasses, with three-point electrooculography (EOG) and six-axis sensors (an accelerometer and a gyroscope) to detect the following actions: blink occurrence, speed, and strength; vertical and horizontal eye movement; and head movement (rotation roll, pitch, and yaw) [26].

Participant's heart rates, (HR) were measured using the Garmin Vivoactive 3 GPS smartwatch with a heart rate sensor located on the back of the device, which displayed the current heart rate in beats per minute (bpm). A graph of the heart rate was also displayed on the device.

\section{Research group}

The research participants were 30 third-, fourth-, and fifth-year students (junior, mid-senior, and senior, respectively) of the Dental Division of Silesian Medical University, Katowice, Poland. Ten students were selected from each year group and randomly classified into two equinumerous subgroups: a Management Anxiety Group (MAG) and an Uncontrolled Anxiety Group (UAG). Participation in the study program was determined by the schedule of didactic classes that coincided with the start date of the experiment. Study subgroups were randomly assigned from a closed pool of tickets marked MAG or $\mathrm{UAG}_{\mathrm{ra}}$

The MAG received a session of PMR and music therapy, detailed and precise jnformation about the task to be performed (analysis of the $3 \mathrm{D}$ model, and measurements of the range, shape, and depth of the tooth's cavity, both without any time limit), and recommendations to fully concentrate on precision shape modeling. Relaxation procedures were guided by an experienced physiotherapist [27]: subjects were placed in a room with dimmed lights and sat in chairs to relax the main muscle groups and relieve tension. Relaxing music was also played from a portable audio system using a CD played at a volume of 50-60 DB.

The UAG was not given any information or recommendations and did not undergot relaxation training. The UAG was given a time limit to prepare the cavity. The procedure was conducted under careful teacher supervision but without assistance. The teacher was tasked with informing the students of the passing time at 10 second intervals as well as evaluating their work using the phrase: 'It's bad, not good, hurry up'[24,25]

\begin{tabular}{|c|c|}
\hline Formatted & $\ldots[368]$ \\
\hline \multicolumn{2}{|l|}{ Deleted: $d$} \\
\hline Formatted & $\ldots[370]$ \\
\hline Deleted: ...nd & $\ldots[371]$ \\
\hline \multicolumn{2}{|l|}{ Deleted: } \\
\hline Formatted & $\ldots[372]$ \\
\hline Formatted & $\ldots[373]$ \\
\hline \multicolumn{2}{|c|}{ Deleted: In this experiment, the } \\
\hline \multicolumn{2}{|l|}{ Deleted: } \\
\hline Formatted & $\ldots[375]$ \\
\hline \multicolumn{2}{|c|}{ Deleted: smartglasses (eye-sensing device for researchyer 8 ) } \\
\hline Formatted & $\ldots[374]$ \\
\hline Formatted & $\ldots[377]$ \\
\hline \multicolumn{2}{|l|}{ Deleted: } \\
\hline Formatted & $\ldots[378]$ \\
\hline \multicolumn{2}{|l|}{ Deleted: } \\
\hline Formatted & $\ldots[379]$ \\
\hline \multicolumn{2}{|l|}{ Deleted: } \\
\hline Formatted & $\ldots[380]$ \\
\hline \multicolumn{2}{|l|}{ Deleted: } \\
\hline Formatted & $\ldots[381]$ \\
\hline \multicolumn{2}{|l|}{ Deleted: } \\
\hline Formatted & $\ldots[382]$ \\
\hline \multicolumn{2}{|l|}{ Deleted: } \\
\hline Formatted & $\ldots[383]$ \\
\hline \multicolumn{2}{|l|}{ Deleted: } \\
\hline Formatted & $\ldots[384]$ \\
\hline \multicolumn{2}{|l|}{ Deleted: } \\
\hline \multicolumn{2}{|l|}{ Deleted: } \\
\hline Formatted & $\ldots[386]$ \\
\hline \multicolumn{2}{|l|}{ Deleted: } \\
\hline \multicolumn{2}{|l|}{ Deleted: sensors } \\
\hline \multicolumn{2}{|l|}{ Deleted: } \\
\hline Formatted & $\ldots[387]$ \\
\hline Formatted & $\ldots[389]$ \\
\hline \multicolumn{2}{|l|}{ Deleted: } \\
\hline Formatted & $\ldots[390]$ \\
\hline \multicolumn{2}{|l|}{ Deleted: } \\
\hline Formatted & $\ldots[391]$ \\
\hline Deleted: & \\
\hline Formatted & $\ldots[385]$ \\
\hline Formatted & $\ldots[388]$ \\
\hline Formatted & $\ldots[392]$ \\
\hline Deleted: & \\
\hline Formatted & $\ldots[393]$ \\
\hline Deleted: & \\
\hline Formatted & $\ldots[394]$ \\
\hline Deleted: & \\
\hline Formatted & $\ldots[395]$ \\
\hline Deleted: & \\
\hline Formatted & $\ldots[396]$ \\
\hline Deleted: & \\
\hline Formatted & $\ldots[397]$ \\
\hline Deleted: & \\
\hline Formatted & $\ldots[398]$ \\
\hline Deleted: & \\
\hline Formatted & $\ldots[399]$ \\
\hline Deleted: & \\
\hline Formatted & $\ldots[400]$ \\
\hline
\end{tabular}


All participants were equipped with a set of monitoring devices (the smart glasses and smartwatch) during the cavity preparation procedure to measure the vital signs that defined their stress intensity.

\section{Experiment}

Students were asked to perform the preparation of a cavity jn a standard phantom molar tooth 36 of a 1:1 scale (Frasaco $\mathrm{GmbH}_{\mathrm{e}}$ Germany) with limited or unlimited time, depending on the group they were assigned to. The matrix with the outline of the cavity was prepared beforehand (Figure 1).

They, used a Round End, Taper NTI Rotary Dental Instruments, burr with head length 4 mm:diameter 009 mm; $_{\text {grit }}$ medium.

The participants performed the procedure with high speed ending ( 350.000 revolutions per minute) and water cooling. A pattern of the cavity outline was presented on a model (Figure 2) prepared using a $3 \mathrm{D}$ printer (Formlabs 2 ). The model was characterized by the following features: magnification $\times 10$, material: Photopolymer Resin Black FLGPBK04, volume: $389.82 \mathrm{ml}$, layer thickness: $0.025 \mathrm{~mm}$, number of layers: 3312 , print time: $57 \mathrm{~h}, 13 \mathrm{~min}$.

After a one-week period, the participants switched experimental groups and performed the same procedure, allowing us to determine the differences between $\mathrm{MAG}$ and $\mathrm{UAG}$ data for the same individual.

Data acquired from the smart glasses worn during the experiment were divided into three parts:

I. electrooculography (EOG),

II. acceleration (ACC),

III. gyroscope (GYRO).

The data collected during the first four seconds of the experiment (when the participant started the procedure) and the last four seconds (when the procedure was completed) were not used for analysis, as the largest change in values were observed during that time.

60 experiments were conducted and one common episode that caused rapid rises of amplitude in a signal was chosen to properly evaluate the results. Consent for the experiments was issued by the Ethical Commission of Medical University of Silesia with resolution number KNW/0022/KB1/79/18 on October 16, 2018. All participants gave their written consent prior to the start of the study

\section{Statistical procedures}

Statistical analysis was performed using Statistica Version 9.0 (MUS Katowice, Poland). Results are presented as mean and standard deviation (SD). Statistical analysis of HR values revealed that all distributions were normally distributed. Differences among groups were analyzed using the Student's t-test. A Shapiro-Wilk test of normality was performed. A one-way analysis of

\begin{tabular}{|c|c|}
\hline Formatted & $\ldots[505]$ \\
\hline \multicolumn{2}{|l|}{ Deleted: } \\
\hline \multicolumn{2}{|l|}{ Deleted: } \\
\hline \multicolumn{2}{|l|}{ Deleted: } \\
\hline Formatted & $\ldots[508]$ \\
\hline Formatted & $\ldots[509]$ \\
\hline \multicolumn{2}{|l|}{ Deleted: } \\
\hline \multicolumn{2}{|l|}{ Deleted: } \\
\hline \multicolumn{2}{|l|}{ Deleted: } \\
\hline Formatted & $\ldots[514]$ \\
\hline \multicolumn{2}{|l|}{ Deleted: } \\
\hline \multicolumn{2}{|c|}{ Deleted: smartglasses } \\
\hline Formatted & $\ldots[507]$ \\
\hline Formatted & $\ldots[510]$ \\
\hline Formatted & $\ldots[511]$ \\
\hline Formatted & $\ldots[512]$ \\
\hline \multicolumn{2}{|l|}{ Deleted: } \\
\hline \multicolumn{2}{|l|}{ Deleted: appliances } \\
\hline Formatted & $\ldots[513]$ \\
\hline Formatted & $\ldots[515]$ \\
\hline Formatted & $\ldots[516]$ \\
\hline Formatted & $\ldots[517]$ \\
\hline \multicolumn{2}{|l|}{ Deleted: } \\
\hline Formatted & $\ldots[518]$ \\
\hline \multicolumn{2}{|l|}{ Deleted: } \\
\hline Formatted & $\ldots[519]$ \\
\hline \multicolumn{2}{|l|}{ Deleted: } \\
\hline Formatted & $\ldots[520]$ \\
\hline \multicolumn{2}{|l|}{ Deleted: } \\
\hline \multicolumn{2}{|l|}{ Deleted: } \\
\hline \multicolumn{2}{|l|}{ Deleted: } \\
\hline Formatted & $\ldots[522]$ \\
\hline \multicolumn{2}{|l|}{ Deleted: in order } \\
\hline Formatted & $\ldots[523]$ \\
\hline Formatted & $\ldots[524]$ \\
\hline \multicolumn{2}{|l|}{ Deleted: control } \\
\hline Formatted & $\ldots[525]$ \\
\hline \multicolumn{2}{|l|}{ Deleted: } \\
\hline Formatted & $\ldots[526]$ \\
\hline Deleted: paramete & \\
\hline Formatted & $\ldots[521]$ \\
\hline Formatted & $\ldots[527]$ \\
\hline Deleted: & \\
\hline Formatted & $\ldots[528]$ \\
\hline Formatted & $\ldots[529]$ \\
\hline Deleted: ...ere ... & xerciss90] \\
\hline Commented [JMS & \\
\hline Formatted & $\ldots[531]$ \\
\hline Deleted: cavity. $\llbracket$ & $\ldots[532]$ \\
\hline Formatted & $\ldots[533]$ \\
\hline Formatted & $\ldots[534]$ \\
\hline Formatted & $\ldots[535]$ \\
\hline Deleted: burr was & \\
\hline Formatted & $\ldots[536]$ \\
\hline Deleted: & \\
\hline Formatted & $\ldots[537]$ \\
\hline Deleted: & \\
\hline Formatted &..$[538]$ \\
\hline Deleted: & \\
\hline
\end{tabular}


variance was used to compare the groups. Fisher's LSD variant was used for two-tailed post hoc tests in justified cases of this test (two cases).

\section{Results}

\section{A. HR Data}

The Student's t-test was used to evaluate the differences in the before and after results from the UAG and MAG groups. Statistically, significant differences were only found in the mid-senior UAG $(\mathrm{p}=0.02)$ (Table 1$)$. The heart rate in this group gradually decreased while performing the dental task, which implies that stress management training is useful for less experienced students.

The ANOVA test was used to compare $H_{2}$ values among the UAG and it revealed significant differences between the year groups (Figure 3). The post hoc test results show that significant differences occurred between the third- and fourth-year groups (Table 2).

The results of the ANOVA test in the UAG show significant differences in HR between year groups. The post hoc test results show significant differences when comparing juniors with midseniors, and mid-seniors and seniors, with mid-seniors. Mid-year seniors had a lower HR than juniors and seniors. There is no statistically significant difference in $\mathrm{HR}$ between juniors and seniors,(Figure 4, Table 3).

No statistically significant results were obtained for the MAG (MAG before: $p=0.69$, MAG after $\mathrm{p}=0.13$ ). The anti-stress training jndicates, that $H R$ values did not differ significantly.

\section{B. Data from EOG}

Data were collected on blinks, squinting of eyes, and probable change of sight directions There were higher amplitudes and more frequent episodes demonstrated by seven participants in the UAG (Figure 5), while their counterparts in the MAG received lower scores (Figure 6). There were more frequent plinks and squints, and higher muscle tension in eyes in the UAG.

The number of episodes of saccades and blinks was determined as the number of local maxima of the moving average of the EOG_H signal. EOG_H was used to find these episodes because, according to the producers of the JINS glasses, the greatest changes in EOG while blinking occur in the horizontal component of this signal. The signal processing steps are as follows:

1. Zero-phase 3rd order band-pass Butterworth filter (frequency range 1-35 Hz). Filtration was performed in MATLAB R2019b using the filtfilt function. The frequency range of the bandpass filter was chosen based on [28].

2. Median filtration using 5 th order median filter.

3. Moving average FIR (finite impulse response) filter (window-width of 45 samples).

4. Finding local maxima using findpeaks function (Figure 7).
Deleted: Differences in the UAG and MAG groups betpreep

Formatted

Deleted: values were analyzed using the t-student test... [643]

Formatted

Deleted: the

Formatted

Deleted: only

Formatted

Deleted: Mid

Formatted

Deleted: group

Formatted

Deleted: Heart

Formatted

Deleted: most

Formatted

Deleted: Table 1. Mean, standard deviation (SD), mediag51]

Formatted

Deleted: obtained

Formatted

Deleted: before

Formatted

Deleted: in

Deleted: groups

Formatted

Formatted

Deleted: years (see

Formatted

Deleted: , in principle, the significant difference concerpp858]

Formatted

Formatted

Formatted

Deleted: years

Formatted

Deleted: state that the

Formatted

Deleted: relate to the comparison of Junior

Formatted

Deleted: Mid-senior

Formatted

Deleted: Mid-senior

Formatted

Deleted: Senior

Formatted

Deleted: Mid-senior

Formatted

Deleted: have

Formatted

Deleted: Junior

Formatted

Deleted: Senior

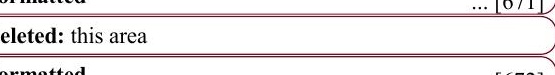


1739 Based on the comparison of the mean number of peaks, mid-senior and senior MAG students had 1740 a lower number of peaks than students from the UAG. The MAG had a higher number of peaks 1741 than the UAG in junior-level students (Table 4).

1742

\section{Data from ACC and GYRO}

Data from the gyroscope were observed as tilts of the head along each plane of the body. Accelerometer data were collected from movements of the, head, and their acceleration and jntensity. The complete information about the dynamics of the head movements was acquired from both the accelerometer and gyroscope.

The average acceleration (ACC) of juniors and seniors in the UAG and MAG on the $\mathrm{x}$ - and $\mathrm{y}$-axes was higher in the UAG than in the MAG, but was lower on the z-axis. The amplitude of the gyration on $\mathrm{x}$-axis head tilts were larger for all students (junior, mid-senior, and senior) in the UAG than for the MAG.

The standard deviations of the head rotation angles were high when considering the average(Table 5).

The ACC X signal was used in further analyses because of the differences in amplitude among all study groups (Figures 8-9).

Each of the students who took part in the examination was added to the histogram. Three ranges were proposed from minimum to maximum $A C C \_X$, with the arousal flagged The mean values of ACC_X signal amplitudes were calculated for each of the students from the UAG and MAG in order to compare his/her stress management abilities (Table 6 ).

The following ranges were determined based on the data:

1. -1509 to -726 : low jevel of arousal;

2. -726 to 57.7: normal jevel of arousal;

3. 57.7 to 841: high level of arousal;

Students were classified as having a particular range of arousal based on their medium values, The greatest number of students were characterized by normal arousal levels in both the UAG and MAG. High jevel of arousal was observed in three participants: two seniors from the UAG and one senior from the MAG (Table 7).

Simultaneous movements of both eyes in the same direction are called saccades. Typical characteristics of saccadic movements are $400^{\circ} \mathrm{s}$ for the maximum velocity, $20_{\mathrm{s}}^{\circ}$ for the amplitude, and $80 \mathrm{~ms}$ for the duration. Fixations are static states of the eyes during which the gaze is held at a specific location [29]. Humans typically alternate saccadic eye movements and fixations while perceiving their environment and the ACC signal and the EOG signal are needed to evaluate these motions.

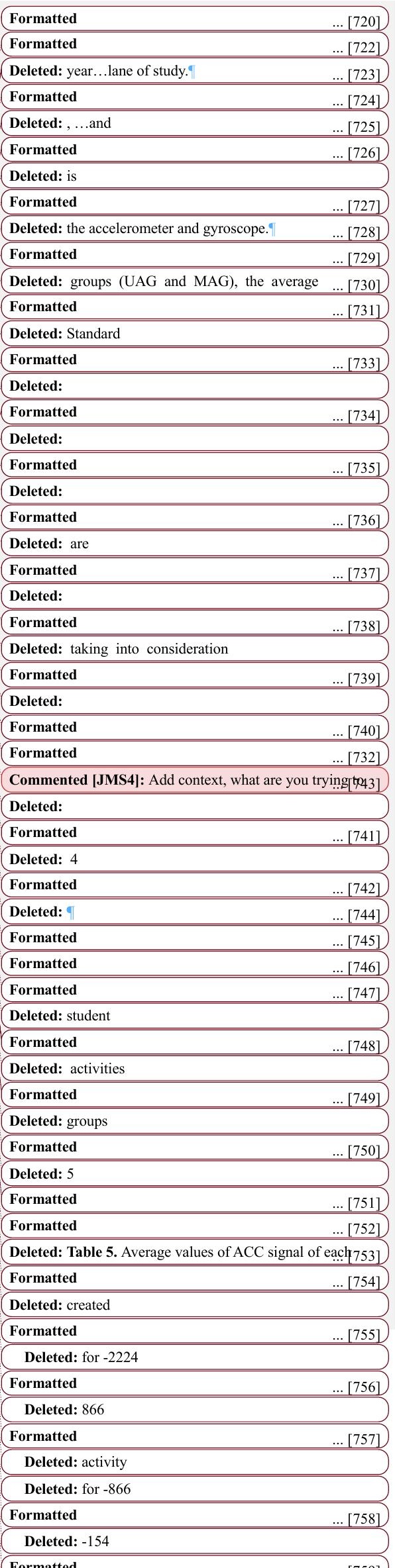


The pilot scale of arousal was created to determine levels of stress, however, its usefulness should be confirmed in further research. There is an arbitrarily measurable difference in ACC and EOG when using JINS glasses and, inversely, eye movements can be tracked by analyzing the changes in the EOG signal. The electrode pairs capture the horizontal and the vertical components of eye motion.

EOG requires good electrode placement on the horizontal and vertical axes of eye motion to prevent crosstalk between both components. Typically, the signal amplitudes resulting from horizontal eye movements are larger than those from vertical movements. Therefore, crosstalk affects the vertical component more severely. Signal crosstalk poses problems for the robust detection of eye movement events and eye gaze tracking for which both components need to be analyzed simultaneously. In the human eye, only a small central region of the retina, known as the fovea, is sensitive enough for most visual tasks, There are differences between the measurements of $\mathrm{HR}$ and gyration in a student who has knowledge of stress and stress-management techniques and a student who does not $\lceil 30]$.

The statistical tests and histograms obtained from data from the JINS glasses and pulse rates enabled us to systematize and determine a scale for the differences caused by stress.

\section{Summary of the results}

We found that stress management training helped one subject from the senior group to progress from a high level of arousal to a low level of arousal and one subject from the mid-senior group moved from the low arousal level to the normal arousal level after training. According to the literature, arousal may be helpful in a high-pressure situation. Body signals resulting from stress, including butterflies in the stomach, increased heart rate, and muscle tension, are associated with an adaptive stress reaction and improved task performance [31]. We found no direct relationship between eye movement and the definition of stress. However, we, were able to demonstrate with JINS glasses that the training had an effect on eye movement $、$ We also found that mid-senior and senior MAG students had a lower number of peaks, which may indicate the effectiveness of antistress training.

\section{Discussion}

According to the literature dental students experience considerable stress and depressive symptoms during their theoretical and hands-on training [1, 5-6, 18]. Dental schools are known for their highly demanding and stressful learning environments and high levels of depression among their students which is more pronounced in women [1].

The most common data collection method to evaluate stress levels are questionnaires, such as the Dental Environment Stress (DES) questionnaire [32-33] or the Depression, Anxiety and Stress Scale (DASS-21) [34]. We sought to determine the usefulness of JINS glasses for the assessment of stress levels among dental students when they perform dental procedures. The experiment was performed using phantom teeth to avoid patient contact and remove the stress

\begin{tabular}{|c|c|}
\hline Formatted & $\ldots[780]$ \\
\hline Deleted: ๆ & $\ldots[782]$ \\
\hline Formatted & $\ldots[783]$ \\
\hline \multicolumn{2}{|c|}{ Deleted: activity } \\
\hline Formatted & $\ldots[784]$ \\
\hline \multicolumn{2}{|c|}{ Deleted: the first step } \\
\hline Formatted & $\ldots[785]$ \\
\hline \multicolumn{2}{|c|}{ Deleted: the level } \\
\hline Formatted & $\ldots[786]$ \\
\hline \multicolumn{2}{|c|}{ Deleted: . Thus, usage of a created activity scale } \\
\hline Formatted & $\ldots[787]$ \\
\hline \multicolumn{2}{|c|}{ Deleted: It has been shown that when using JINS glasses788] } \\
\hline Formatted & $\ldots[789]$ \\
\hline \multicolumn{2}{|c|}{ Deleted: . Inversely } \\
\hline Formatted & $\ldots[790]$ \\
\hline \multicolumn{2}{|c|}{ Deleted: these } \\
\hline Formatted & $\ldots[791]$ \\
\hline \multicolumn{2}{|c|}{ Deleted: component } \\
\hline Formatted & $\ldots[792]$ \\
\hline \multicolumn{2}{|l|}{ Deleted: } \\
\hline Formatted & $\ldots[793]$ \\
\hline Formatted & $\ldots[794]$ \\
\hline \multicolumn{2}{|c|}{ Deleted: , i. e. } \\
\hline Formatted & $\ldots[795]$ \\
\hline \multicolumn{2}{|c|}{ Deleted: eyes' } \\
\hline Formatted & $\ldots[796]$ \\
\hline \multicolumn{2}{|c|}{ Deleted: , as otherwise increased } \\
\hline Formatted & $\ldots[797]$ \\
\hline \multicolumn{2}{|c|}{ Deleted: occurs } \\
\hline Formatted & $\ldots[798]$ \\
\hline \multicolumn{2}{|l|}{ Deleted: } \\
\hline Formatted & $\ldots[799]$ \\
\hline \multicolumn{2}{|l|}{ Deleted: on } \\
\hline Formatted & $\ldots[800]$ \\
\hline \multicolumn{2}{|c|}{ Deleted: , but } \\
\hline Formatted & $\ldots[801]$ \\
\hline \multicolumn{2}{|c|}{ Deleted: between } \\
\hline Formatted & $\ldots[802]$ \\
\hline \multicolumn{2}{|c|}{ Deleted: how to neutralize it, } \\
\hline Formatted & $\ldots[803]$ \\
\hline Deleted: hay & $\ldots$ [804] \\
\hline Formatted & $\ldots[805]$ \\
\hline Formatted & $\ldots[806]$ \\
\hline Formatted & $\ldots[807]$ \\
\hline \multicolumn{2}{|c|}{ Deleted: Despite the small sample, this study demonstrated } \\
\hline Formatted & $\ldots[808]$ \\
\hline Deleted: ant & \\
\hline Formatted & $\ldots[809]$ \\
\hline Deleted: per & \\
\hline Formatted & $\ldots[810]$ \\
\hline Deleted: the & \\
\hline Formatted & $\ldots[811]$ \\
\hline Deleted: act & $\ldots[812]$ \\
\hline Formatted & $\ldots[813]$ \\
\hline Deleted: foc & edupg.4] \\
\hline Formatted & $\ldots[815]$ \\
\hline Deleted: no1 & tivit816] \\
\hline Formatted & $\ldots[817]$ \\
\hline
\end{tabular}


variable of the subjects' awareness of working on live patients. The student's stress was related to the time restriction and the need to precisely perform the dental procedure. The usefulness of the tools (the smart glasses and the smartwatch) was assessed to measure objective symptoms and to control the vital parameters that confirmed anxiety and stress,

The JINS glasses have been used for the objective evaluation of physical parameters directly related to the intensification of stress [23]. We used JINS glasses for the first time with dental school students during their performance of a task to evaluate and obtain data for analysis and further classification.

There are few reports in the literature on tools used for measuring and analyzing the objective responses of the body to increasing levels of stress and only one pilot study on the subject concerning the area of dentistry. Hunasgi et al. (2018) used the sensor and software located in smartphones to record and analyze the heart rate, oxygen saturation ${ }_{2}$ and stress in dental students before and after their university practical exam [35].

1. Our own pilot study was conducted using a phantom clinical simulation. The least experienced students felt the highest level of stress while fifth year students who had worked with living patients, managed their stress better. These results are consistent with the literature $[3,13$, 16]. which jndicates that clinical work with a living patient is more stressful than the first contact with mechanical dental equipment and the precise preparation of the cavity in a phantom tooth, despite having additional training or experience.

Our, results are similar, to other studies that also had a limited number of participants and data, and reveal that electronic tools with software and sensors can be used to detect stress levels, at any point of time. The JINS glasses were calibrated in preclinical operating conditions and the graphs were analyzed to preliminarily determine the readings that corresponded with a particular stage of concentration or stress. We intend to continue this study by assessing the stress levels of large groups of students performing dental procedures, including students practicing on phantom teeth and later on patients, We will compare the results to the patterns obtained jn this preliminary experiment.

\section{Conclusion}

Our pilot study shows that electronic tools, such as the JINS glasses with software and sensors $_{2}$ can measure different levels of stress experienced by dental students in preclinical operating conditions. We currently lack a clear definition of the states of stress. However, research using modern wearable devices can record baselines and establish patterns of stresses to allow for accurate assessments and management of stress in the future.

Future research should seek to determine whether the prevalence of severe depressive symptoms among students in different disciplines is due to the innate nature of the discipline, the type of curriculum being studied, or the forms of knowledge and manual skills required.

\begin{tabular}{|c|c|}
\hline Formatted & $\ldots[849]$ \\
\hline Formatted &.. [851] \\
\hline \multicolumn{2}{|c|}{ Deleted: phantom teeth, not necessitating any contac852] } \\
\hline \multicolumn{2}{|l|}{ Formatted } \\
\hline \multicolumn{2}{|c|}{ Deleted: The participants were thus deprived of the [854] } \\
\hline \multicolumn{2}{|l|}{ Deleted: } \\
\hline \multicolumn{2}{|l|}{ Formatted } \\
\hline \multicolumn{2}{|l|}{ Formatted } \\
\hline \multicolumn{2}{|l|}{ Deleted: } \\
\hline \multicolumn{2}{|l|}{ Deleted: } \\
\hline \multicolumn{2}{|l|}{ Formatted } \\
\hline \multicolumn{2}{|l|}{ Deleted: } \\
\hline \multicolumn{2}{|l|}{ Deleted: } \\
\hline \multicolumn{2}{|l|}{ Deleted: } \\
\hline Formatted &..$[857]$ \\
\hline \multicolumn{2}{|c|}{ Deleted: pressure of } \\
\hline \multicolumn{2}{|l|}{ Deleted: } \\
\hline Formatted & $. . .858]$ \\
\hline \multicolumn{2}{|l|}{ Deleted: } \\
\hline \multicolumn{2}{|l|}{ Formatted } \\
\hline \multicolumn{2}{|l|}{ Formatted } \\
\hline \multicolumn{2}{|l|}{ Formatted } \\
\hline \multicolumn{2}{|l|}{ Formatted } \\
\hline Formatted & $. . .8664]$ \\
\hline Deleted: der & was865] \\
\hline Formatted &..$[866]$ \\
\hline Deleted: & \\
\hline Formatted & $. . .867]$ \\
\hline Deleted: & \\
\hline Deleted: use & \\
\hline Formatted & .. [869] \\
\hline Deleted: & \\
\hline Formatted & $\ldots[870]$ \\
\hline Deleted: & \\
\hline Formatted & $\ldots[871]$ \\
\hline Deleted: & \\
\hline Formatted & $\ldots[872]$ \\
\hline Deleted: & \\
\hline Formatted & $\ldots[868]$ \\
\hline Formatted & $\ldots[873]$ \\
\hline Commented & ling881] \\
\hline Deleted: & \\
\hline Formatted & $\ldots[874]$ \\
\hline Deleted: & \\
\hline Formatted & $\ldots[875]$ \\
\hline Deleted: & \\
\hline Formatted & [876] \\
\hline Deleted: cor & \\
\hline Formatted & .. [877] \\
\hline Deleted: & \\
\hline Formatted & $\ldots[878]$ \\
\hline Deleted: & \\
\hline Formatted & $\ldots[879]$ \\
\hline Deleted: & \\
\hline Formatted & $\ldots[880]$ \\
\hline Deleted: The & \\
\hline Formatted & [882] \\
\hline Deleted: we & \\
\hline Formatted & [883] \\
\hline
\end{tabular}




\section{Acknowledgements}

The authors wish to thank all of the students who participated jin this study.

\section{References}

1. Al Faris E, Farhana IF, Qureshi R, Naeem N, Alshomrani A, Ponnamperuma G, Al Yousufi

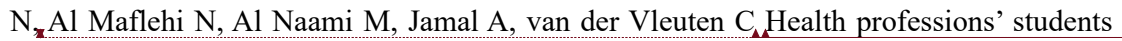
have an alarming prevalence of depressive symptoms: exploration of the associated factors. BMC Med Educ. 2016;16:279-286. Published online 2016 Oct 21. doi: 10.1186/s12909016-0794-y

2. Rada RE, Johnson-Leong C. Stress, burnout, anxiety and depression among dentists. $J$ Am Dent Assoc. 2004;135(6):788-794.

3. Piazza-Waggoner $\mathrm{CA}$, Cohen LL, Kohli K, Taylor BK. Stress management for dental students performing their first pediatric restorative procedure. $J$ Dent Educ. 2003;67(5):542-548.

4. Harazin WE, Allison PJ, Kumar,AR, Mancini L, Lambrou, A, Bedos C. Asystematic review of stress in dental students. J Dent Educ. 2014;78(2);226-242.

5. Farokh-Gisour E, Hatamvand M. Investigation of stress level among dentistry students, general dentists, and pediatric dental specialists during performing pediatric dentistry in Kerman, Iran, in 2017. Open Dent. 2018;12:631-637.

6. Chandrasekaran B, Cugati N, Kumaresan R. Dental students' perception and anxiety levels during their first local anesthetic injection. Malays JMed Sci. 2014;21(6):45-51.

7. Martinez R, Irigoyen E, Arruti A, Martin JI, Muguerza J. A real-time stress classification system based on arousal analysis of the nervous system by an F-state machine. Comput Methods and Programs Biomed. 2017; 148: 81-90

8. Schachner S, Singer JE. Cognitive, social and physiological determinants of emotional state in: WG Parrott (ED). Emotion in Social Psychology: Essential Readings.2000: 76-93

9. Dyrye LN, Thomas MR, Shanafelt TD. Systematic review of depression, anxiety, and other jndicators of psychological distress among U.S. and Canadian medical students. Acad Med. 2006;81(4):354-373

10. Mohannad A, Albajjar A, Marvan A, Bakrman R. Prevalence and correlates of depression among male medical students and interns in Albaha University, Saudi Arabia. J Family Med Prim Care. 2019; 8(6): 1889-1894.

11. Brazeau CM, Shanafelt T, Durning SJ, Massie FS, Eacker A, Moutier C, Satele DV, Sloan

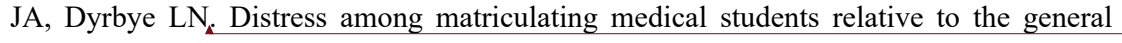
population. Acad Med. 2014;89:1520-1525.

\begin{tabular}{|c|c|}
\hline Formatted & $\ldots[988]$ \\
\hline Deleted: ๆ & $\ldots[990]$ \\
\hline Formatted & $\ldots[991]$ \\
\hline \multicolumn{2}{|l|}{ Deleted: } \\
\hline \multicolumn{2}{|l|}{ Deleted: } \\
\hline \multicolumn{2}{|l|}{ Deleted: } \\
\hline \multicolumn{2}{|l|}{ Deleted: } \\
\hline Formatted & $\ldots[992]$ \\
\hline \multicolumn{2}{|l|}{ Deleted: } \\
\hline \multicolumn{2}{|l|}{ Deleted: } \\
\hline Formatted & $\ldots[994]$ \\
\hline \multicolumn{2}{|l|}{ Deleted: } \\
\hline \multicolumn{2}{|l|}{ Deleted: } \\
\hline Formatted & $\ldots[993]$ \\
\hline Formatted & $\ldots[995]$ \\
\hline Formatted & $\ldots[996]$ \\
\hline Formatted & $\ldots[997]$ \\
\hline Formatted & ... [998] \\
\hline Formatted & $\ldots[999]$ \\
\hline Formatted & $\ldots[1000]$ \\
\hline Formatted & $\ldots[1001]$ \\
\hline \multicolumn{2}{|l|}{ Deleted: ๆ } \\
\hline Formatted & $\ldots[1002]$ \\
\hline \multicolumn{2}{|l|}{ Deleted: } \\
\hline Formatted & $\ldots[1003]$ \\
\hline \multicolumn{2}{|l|}{ Deleted: } \\
\hline Formatted & $\ldots[1004]$ \\
\hline Field Code Changed & $\ldots[1005]$ \\
\hline Formatted & $\ldots[1006]$ \\
\hline \multicolumn{2}{|l|}{ Deleted: } \\
\hline Formatted & $\ldots[1007]$ \\
\hline Formatted & $\ldots[1008]$ \\
\hline \multicolumn{2}{|l|}{ Deleted: } \\
\hline Formatted & $\ldots[1009]$ \\
\hline \multicolumn{2}{|l|}{ Deleted: } \\
\hline Formatted & $\ldots[1010]$ \\
\hline \multicolumn{2}{|l|}{ Deleted: } \\
\hline Formatted & $\ldots[1011]$ \\
\hline \multicolumn{2}{|l|}{ Deleted: } \\
\hline Formatted & $\ldots[1012]$ \\
\hline \multicolumn{2}{|l|}{ Deleted: } \\
\hline Formatted & $\ldots[1013]$ \\
\hline \multicolumn{2}{|l|}{ Deleted: } \\
\hline Formatted & $\ldots[1014]$ \\
\hline \multicolumn{2}{|l|}{ Deleted: } \\
\hline Formatted & $\ldots[1015]$ \\
\hline Deleted: & \\
\hline Formatted & $\ldots[1016]$ \\
\hline Deleted: dentist. & \\
\hline Formatted & $\ldots[1017]$ \\
\hline Deleted: & \\
\hline Formatted & $\ldots[1018]$ \\
\hline Deleted: & \\
\hline Formatted & $\ldots[1019]$ \\
\hline Deleted: & \\
\hline Formatted & $\ldots[1020]$ \\
\hline Deleted: & \\
\hline Formatted & $\ldots[1021]$ \\
\hline Formatted & $\ldots[1022]$ \\
\hline
\end{tabular}


12. Lugassy D, Levanon Y, Shpack N, Levartovsky S, Pilo R, Brosh T. An interventional study for improving the manual dexterity of dentistry students. PLoS One. 2019; 14(2): e0211639. doi: 10.1371/journal.pone.0211639.

13. Ali $\mathrm{K}_{\mathrm{s}}$ Cockerill $\mathrm{J}_{\mathrm{B}}$ Zahra $\mathrm{D}_{\mathrm{B}}$. Tredwin $\mathrm{C}_{R}$ Ferguson $\mathrm{C}$. Impact of progress testing on the learning experiences of students in medicine, dentistry and dental therapy. BMC Med Educ. 2018;18(1): 253. doi: 10.1186/s12909-018-1357-1.

14. Abu-Ghazaleh $\mathrm{SB}_{\text {S }}$ Sonbol $\mathrm{HN}_{\mathrm{R}}$ Rajab LD. A longitudinal study of psychological stress among undergraduate dental students at the University of Jordan. BMC Med Educ. 2016;16: 90. doi: 10.1186/s12909-016-0612-6.

15. Roberts LW. Understanding depression and distress among medical students. JAMA. 2010;304:1231-1233.

16. Davidovich E, Pessov Y, Baniel A, Ram D. Levels of stress among general practitioners, students and specialists in pediatric dentistry during dental treatment. J Clin Pediatr Dent. 2015;39(5): 419422. doi: 10.17796/1053-4628-39.5.419.

17. Kiesre J, Herbison P. Clinical anxieties among dental students. NZ Dent J. 2000;96(426): 138-139.

18. Elani HW, Allison PJ, Kumar RA, Mancini L, Lambrou A, Bedos C. A systematic review of stress in dental students. J Dent Educ, 2014;78(2): 226-242.

19. Labrague IJ, McEnroe-Petitte DM, Gloe D, Thomas L, Papathanasiou IV, Tsaras K. A literature review on stress and coping strategies in nursing students. J. Ment. Health. 2017;26 (5): 471-480.doi: 10.1080/09638237.2016.1244721.

20. Ince S, Cevik K. The effect of listening to music about the anxiety of the nursing students during their first blood extraction experience. Indian J. Physiol. Pharmacol. 2014; 58 (3): 298-301.

21. Hashim HA, Zainol NA. Changes in emotional distress, short term memory, and sustained attention following 6 and 12 sessions of progressive muscle relaxation training in 10-11 years old primary school children. Psychol. Health Med. 2015; 20 (5): 623-628. doi: $\underline{10.1080 / 13548506.2014 .1002851 .}$

22. Ishimaru S, Kunze K, Tanaka K, Uema Y, Kise K, Inami M. Smart eyewear for interaction andactivity recognition. In: $\mathrm{CHI}$ EA ' 15 Proceedings of the 33rd Annual ACM Conference on Human Factors in Computing Systems; NewYork, NY, USA, April 18-23, 2015: 307-310. doi: $10.1145 / 2702613.2725449$.

23. Ogawa T, Takahashi M, Kawashima R. Human cognitive control mode estimation using JINS MEME. IFAC-Papersonline. 2016;49(19): 331-336. doi: 10.1016/j.ifacol. 2016.10.571.xc

24. Dedovic K, Renwick R, Mahami NK, Engert V, Lupien SJ, Pruessner JC. The Montreal Imaging Stress Task: using functional imaging to investigate the effects of perceiving and

\begin{tabular}{|c|c|}
\hline Formatted & $\ldots[1150]$ \\
\hline \multicolumn{2}{|c|}{ Deleted: <\#> Lugassy D, Levanon Y, Shpack N, ... [1152] } \\
\hline \multicolumn{2}{|c|}{ Deleted: Cockerill J } \\
\hline Formatted & $\ldots[1156]$ \\
\hline \multicolumn{2}{|c|}{ Deleted: Zahra D } \\
\hline Formatted & $\ldots[1153]$ \\
\hline Formatted & $\ldots[1154]$ \\
\hline \multicolumn{2}{|c|}{ Deleted: Ali K } \\
\hline \multicolumn{2}{|c|}{ Deleted: Tredwin C } \\
\hline \multicolumn{2}{|c|}{ Deleted: Ferguson C. } \\
\hline Formatted & $\ldots[1155]$ \\
\hline Formatted & $\ldots$ [1157] \\
\hline Formatted & $\ldots[1158]$ \\
\hline Formatted & $\ldots[1159]$ \\
\hline \multicolumn{2}{|c|}{ Deleted: BMC $\rightarrow$ Med Educ. } \\
\hline Formatted & $\ldots[1160]$ \\
\hline \multicolumn{2}{|c|}{ Deleted: Abu-Ghazaleh SB } \\
\hline Formatted & $\ldots[1161]$ \\
\hline \multicolumn{2}{|c|}{ Deleted: Sonbol HN } \\
\hline Formatted & $\ldots[1162]$ \\
\hline \multicolumn{2}{|c|}{ Deleted: Rajab LD. } \\
\hline Formatted & $\ldots[1163]$ \\
\hline \multicolumn{2}{|c|}{ Deleted: stressamong } \\
\hline Formatted & $\ldots[1164]$ \\
\hline \multicolumn{2}{|c|}{ Deleted: BMC Med Educ. } \\
\hline Formatted & $\ldots[1165]$ \\
\hline Formatted & $\ldots[1166]$ \\
\hline Formatted & $\ldots[1167]$ \\
\hline \multicolumn{2}{|c|}{ Deleted: Pessov Y, Baniel A, Ram D. } \\
\hline Formatted & $\ldots[1168]$ \\
\hline Formatted & $\ldots[1169]$ \\
\hline \multicolumn{2}{|l|}{ Deleted: } \\
\hline Formatted & $\ldots[1170]$ \\
\hline \multicolumn{2}{|l|}{ Deleted: } \\
\hline Formatted & $\ldots[1171]$ \\
\hline Formatted & $\ldots[1172]$ \\
\hline Formatted & $\ldots[1173]$ \\
\hline Formatted & $\ldots[1174]$ \\
\hline \multicolumn{2}{|l|}{ Deleted: } \\
\hline Formatted & $\ldots[1175]$ \\
\hline \multicolumn{2}{|l|}{ Deleted: } \\
\hline Formatted & $\ldots[1176]$ \\
\hline \multicolumn{2}{|l|}{ Deleted: } \\
\hline Formatted & $\ldots[1177]$ \\
\hline \multicolumn{2}{|l|}{ Deleted: } \\
\hline Formatted & $\ldots[1178]$ \\
\hline \multicolumn{2}{|l|}{ Deleted: } \\
\hline Formatted & $\ldots[1179]$ \\
\hline \multicolumn{2}{|l|}{ Deleted: } \\
\hline Formatted & $\ldots[1180]$ \\
\hline Deleted: & \\
\hline Formatted & $\ldots[1181]$ \\
\hline Formatted & $\ldots[1182]$ \\
\hline Formatted & $\ldots[1151]$ \\
\hline
\end{tabular}


processing psychosocial stress in the human brain. JPsychiatry Neurosci 2005; 30(5):319$\underline{325 .}$

25. Ghazali DA, Darmian-Rafei I, Nadolny J, Sosner P, Ragot S, Oriot D. Evaluation of stress response using psychological, biological and elektrophysiological markers during immersive simulation of life threatening events in multidisciplinary teams. Aust Crit Care. 2018; 31:226-233

26. Chen L, Hoey J, Nugent CD, Cook DJ, Yu Z. Sensor-based activity recognition. IEEE Transactions on Systems, Man, and Cybernetics Part C (Applications and Review). 2012;42(6): 790-808. doi: 10.1109/TSMCC.2012.2198883

27. Gallego-Gómez JI, Balanza S, Leal-Liopis J, García-Méndez JA, Oliva-Pérez J, DoménechTortosa J, Gómez-Gallego M, Simonelli-Muñoz AJ, Rivera-Caravaca JM. Effectiveness of music therapy and progressive muscle relaxation in reducing stress before exams and improving academic performance in Nursing students: A randomized trial. Nurse Educ Today. 2020; 84: 104217. doi:10.1016/j.ned t.2019.104217

28. Nor'aini A.J., Raveendran P., Selvanathan N. A Comparative Analysis of Zernike moments and Principal Component Analysis as Feature extractors for Face Recognition. In: Ibrahim F., Osman N.A.A., Usman J., Kadri N.A. (eds) 3rd Kuala Lumpur International Conference on Biomedical Engineering 2006. IFMBE Proceedings, 2007, vol. 15, pp. 37-41. Springer, Berlin, Heidelberg. doi: 10.1007/978-3-540-68017-8 11

29. Cognolato M, Graziani M, Giordaniello F, Saetta G, Bassetto F, Brugger P, Caputo B, Müller H, Atzori M. Semi-automatic training of an object recognition system in scene camera data using gaze tracking and accelerometers. Computer Vision Systems : 11th International Conference, ICVS 2017, Shenzhen, China, 2017; July 10-13. doi: $\underline{10.1007 / 978-3-319-68345-4 \_16}$

30. Bulling A, Roggen D, Tröster G.. It's in Your Eyes: Towards Context-Awareness and Mobile HCI Using Wearable EOG Goggles. 10th international Conference on Ubiquitous Computing, UbiComp. 2008;344 :84-93. doi:10.1145/1409635.1409647.

31. Jamieson JP, Peters BJ, Greenwood EJ, Altose AJ. Reappraising stress arousal improves performance and reduces evaluation anxiety in classroom exam situations. Soc Psychol Pers Sci. 2016;7(6): 579-587. doi: 10.1177/ 1948550616644656

32. Manolova MS, Stefanova VP, Manchorova-Veleva NA, Panavotov IV, Levallois B, TraminiP, Orti V. A Five-year Comparative Study of Perceived Stress Among Dental Students at Two European Faculties. Folia Med. 2019;61(1): 134-142. doi: 10.2478/folmed2018-0052.

Formatted: Default Paragraph Font, Font color: Black
Formatted: Normal, Space After: 8 pt, Line spacing:
Multiple 1.15 li, Outline numbered + Level: 1 + Numbering
Style: $1,2,3, \ldots+$ Start at: 1 + Alignment: Left + Aligned
at: $0.25 "+$ Indent at: 0.5", Adjust space between Asian text
and numbers, Font Alignment: Auto, Pattern: Clear (White)
$\begin{aligned} & \text { Formatted: Default Paragraph Font, Font: Not Italic, Font } \\ & \text { color: Black }\end{aligned}$
Deleted:
Formatted: Default Paragraph Font, Font: Not Italic, Font
color: Black
Formatted: Default Paragraph Font, Font color: Black

Formatted: Font: 12 pt, Font color: Black Formatted: Normal, Space After: 8 pt, Line spacing: Multiple 1.15 li, Outline numbered + Level: $1+$ Numbering Style: $1,2,3, \ldots+$ Start at: $1+$ Alignment: Left + Aligned at: $0.25^{\prime \prime}+$ Indent at: $0.5^{\prime \prime}$, Adjust space between Asian text and numbers, Font Alignment: Auto, Pattern: Clear (White)

Formatted: Normal, Tab stops: 6.26 ", Right 
Formatted: Normal, Tab stops: 6.26", Right

33. Babar MG, Hasan SS, Ooi YJ, Ahmed SI, Wong PS, Ahmad SF, Mnm-Rosdy NM, Malik NA. Perceived sources of stress among Malaysian dental students. J Med Educ. 2015; 2(6): 56-61. doi: 10.5116/ijme.5521.3b2d.

34. Basudan S, Bnanzan N, Alhassan A. Depression, anxiety and stress in dental students. Int J Med Educ. 2017;24(8): 179-186. doi: 10.5116/ijme.5910.b961.

35. Hunasgi S, Koneru A, Rudraraju A, Manvikar V, Vanishree M. Stress recognition in dental students using smartphone sensor and a software: A pilot study. J Oral Maxillofac Pathol. 2018; 22(3): 314-317. doi: 10.4103/jomfp.JOMFP_168_18
Formatted: Font: 12 pt, Font color: Black

Formatted: Font: 12 pt, Font color: Black

Formatted: Font: 12 pt, Font color: Black Deleted: J Oral Maxillofac Pathol.

Formatted: Font: 12 pt, Font color: Black

Formatted: Font color: Black

Formatted: English (US)

Formatted: Line spacing: Multiple $1.15 \mathrm{li}$ 


\begin{tabular}{|c|c|c|}
\hline Page 1: [1] Formatted & KPachonska & 3/17/20 2:15:00 PM \\
\hline \multicolumn{3}{|c|}{ Normal, Tab stops: $6.26 "$, Right } \\
\hline Page 1: [2] Formatted & KPachonska & 3/17/20 2:15:00 PM \\
\hline \multicolumn{3}{|c|}{ Normal, Tab stops: 6.26", Right } \\
\hline Page 1: [3] Style Definition & KPachonska & 3/17/20 2:15:00 PM \\
\hline \multicolumn{3}{|l|}{ Table Caption } \\
\hline Page 1: [4] Style Definition & KPachonska & 3/17/20 2:15:00 PM \\
\hline
\end{tabular}

Balloon Text: English (US), Border: : (No border), Border: Top: (No border), Bottom: (No border), Left:

(No border), Right: (No border), Between : (No border), Bar : (No border)

\begin{tabular}{|lrl}
\hline Page 1: [5] Style Definition & KPachonska & 3/17/20 2:15:00 PM \\
\hline
\end{tabular}

Comment Subject: English (US), Border: : (No border), Border: Top: (No border), Bottom: (No border),

Left: (No border), Right: (No border), Between : (No border), Bar : (No border)

Page 1: [6] Style Definition KPachonska

3/17/20 2:15:00 PM

Normal (Web): Font: Border: : (No border), Border: Top: (No border), Bottom: (No border), Left: (No border), Right: (No border), Between : (No border), Bar : (No border)

\begin{tabular}{|lrl}
\hline Page 1: [7] Style Definition & KPachonska & 3/17/20 2:15:00 PM
\end{tabular}

List Paragraph: Font: Border: : (No border), Border: Top: (No border), Bottom: (No border), Left: (No border), Right: (No border), Between : (No border), Bar : (No border)

\begin{tabular}{|lrl}
\hline Page 1: [8] Style Definition & KPachonska & 3/17/20 2:15:00 PM \\
\hline
\end{tabular}

No Spacing: Font: Border: : (No border), Border: Top: (No border), Bottom: (No border), Left: (No border), Right: (No border), Between : (No border), Bar : (No border)

\begin{tabular}{|lrr|}
\hline Page 1: [9] Style Definition & KPachonska & 3/17/20 2:15:00 PM \\
\hline
\end{tabular}

Normal1: Font: Border: : (No border), Border: Top: (No border), Bottom: (No border), Left: (No border), Right: (No border), Between : (No border), Bar : (No border)

\begin{tabular}{|lll}
\hline Page 1: [10] Style Definition & KPachonska & 3/17/20 2:15:00 PM
\end{tabular}

Standard: Font: Border: : (No border), Border: Top: (No border), Bottom: (No border), Left: (No border), Right: (No border), Between : (No border), Bar : (No border)

\begin{tabular}{|c|c|c|}
\hline Page 1: [11] Style Definition & KPachonska & 3/17/20 2:15:00 PM \\
\hline \multicolumn{3}{|c|}{$\begin{array}{l}\text { Nagłówek i stopka: Font: English (US), Border: : (No border), No widow/orphan control, Border: Top: } \\
\text { (No border), Bottom: (No border), Left: (No border), Right: (No border), Between : (No border), Bar : } \\
\text { (No border) }\end{array}$} \\
\hline Page 1: [12] Style Definition & KPachonska & 3/17/20 2:15:00 PM \\
\hline \multicolumn{3}{|l|}{ Comment Reference } \\
\hline Page 1: [13] Style Definition & KPachonska & 3/17/20 2:15:00 PM \\
\hline \multicolumn{3}{|l|}{ Hyperlink.4 } \\
\hline Page 1: [14] Style Definition & KPachonska & 3/17/20 2:15:00 PM \\
\hline \multicolumn{3}{|l|}{ Hyperlink.3 } \\
\hline Page 1: [15] Style Definition & KPachonska & 3/17/20 2:15:00 PM \\
\hline \multicolumn{3}{|l|}{ Hyperlink.2 } \\
\hline Page 1: [16] Style Definition & KPachonska & 3/17/20 2:15:00 PM \\
\hline \multicolumn{3}{|l|}{ Hyperlink.1 } \\
\hline Page 1: [17] Style Definition & KPachonska & 3/17/20 2:15:00 PM \\
\hline
\end{tabular}


Hyperlink.0

Page 1: [18] Style Definition

KPachonska

3/17/20 2:15:00 PM

Normal: English (US), Border: : (No border), Border: Top: (No border), Bottom: (No border), Left: (No

border), Right: (No border), Between : (No border), Bar : (No border)

\begin{tabular}{|lll|}
\hline Page 1: $[19]$ Deleted & KPachonska & $3 / 17 / 20$ 2:15:00 PM \\
\hline Page 1: $[20]$ Formatted & KPachonska & $3 / 17 / 20$ 2:15:00 PM \\
\hline From text: $0.2 "$, Numbering: Continuous & & $3 / 17 / 202: 15: 00$ PM \\
\hline Page 1: $[21]$ Formatted & KPachonska & \\
\hline Font: $12 \mathrm{pt}$ & & $3 / 17 / 202: 15: 00$ PM \\
\hline Page 1: $[22]$ Formatted & KPachonska & \\
\hline
\end{tabular}

Normal, Space After: 8 pt, Line spacing: Multiple 1.15 li, Pattern: Clear (White)

Page 1: [23] Formatted

KPachonska

3/17/20 2:15:00 PM

Font: Times New Roman, 12 pt

Page 1: [23] Formatted

KPachonska

3/17/20 2:15:00 PM

Font: Times New Roman, $12 \mathrm{pt}$

Page 1: [24] Formatted

KPachonska

3/17/20 2:15:00 PM

Font: $12 \mathrm{pt}$

Page 1: [25] Formatted

KPachonska

3/17/20 2:15:00 PM

Font: $12 \mathrm{pt}$

Page 1: [26] Formatted

KPachonska

3/17/20 2:15:00 PM

Font: $12 \mathrm{pt}$

Page 1: [27] Formatted

KPachonska

3/17/20 2:15:00 PM

Font: $12 \mathrm{pt}$

Page 1: [28] Formatted

KPachonska

3/17/20 2:15:00 PM

Font: $12 \mathrm{pt}$

Page 1: [29] Formatted

KPachonska

3/17/20 2:15:00 PM

Font: $12 \mathrm{pt}$

Page 1: [30] Formatted

KPachonska

3/17/20 2:15:00 PM

Font: $12 \mathrm{pt}$

Page 1: [31] Formatted

KPachonska

3/17/20 2:15:00 PM

Font: $12 \mathrm{pt}$

Page 1: [32] Formatted

KPachonska

3/17/20 2:15:00 PM

Font: $12 \mathrm{pt}$

\begin{tabular}{|lcc|}
\hline Page 1: $[33]$ Formatted & KPachonska & $3 / 17 / 20$ 2:15:00 PM \\
\hline Font: $12 \mathrm{pt}$ & & $3 / 17 / 20$ 2:15:00 PM \\
\hline Page 1: $[34]$ Formatted & KPachonska & \\
\hline Font: $12 \mathrm{pt}$ & & $3 / 17 / 202: 15: 00$ PM \\
\hline Page 1: $[35]$ Formatted & KPachonska \\
\hline Font: $12 \mathrm{pt}$ & & $3 / 17 / 202: 15: 00$ PM \\
\hline Page 1: $[36]$ Formatted & KPachonska & 3 \\
\hline
\end{tabular}


Font: $12 \mathrm{pt}$

Page 1: [37] Formatted

KPachonska

3/17/20 2:15:00 PM

Font: $12 \mathrm{pt}$

Page 1: [38] Formatted

KPachonska

3/17/20 2:15:00 PM

Font: $12 \mathrm{pt}$

Page 1: [39] Formatted

KPachonska

3/17/20 2:15:00 PM

Font: $12 \mathrm{pt}$

Page 1: [40] Change

Unknown

Field Code Changed

Page 1: [41] Formatted

KPachonska

3/17/20 2:15:00 PM

Font: $12 \mathrm{pt}$

Page 1: [41] Formatted

KPachonska

3/17/20 2:15:00 PM

Font: $12 \mathrm{pt}$

Page 1: [42] Formatted

KPachonska

3/17/20 2:15:00 PM

Font: 14 pt, Bold

Page 1: [43] Formatted

KPachonska

3/17/20 2:15:00 PM

Normal, Space After: 8 pt, Line spacing: Multiple 1.15 li, Pattern: Clear (White)

Page 1: [44] Formatted

KPachonska

3/17/20 2:15:00 PM

Normal, Tab stops: 6.26", Right

Page 1: [45] Formatted

KPachonska

3/17/20 2:15:00 PM

Normal, Tab stops: 6.26", Right

Page 2: [46] Deleted

KPachonska

3/17/20 2:15:00 PM

(

Page 2: [46] Deleted

KPachonska

3/17/20 2:15:00 PM

Page 2: [46] Deleted

KPachonska

3/17/20 2:15:00 PM

$\checkmark$

Page 2: [46] Deleted

KPachonska

3/17/20 2:15:00 PM

$\checkmark$

Page 2: [46] Deleted

KPachonska

3/17/20 2:15:00 PM

$\checkmark$

Page 2: [46] Deleted

KPachonska

3/17/20 2:15:00 PM 


\begin{tabular}{|c|c|c|}
\hline Page 2: [46] Deleted & KPachonska & 3/17/20 2:15:00 PM \\
\hline $\mathrm{v}$ & & \\
\hline \multicolumn{3}{|l|}{.......... } \\
\hline Page 2: [46] Deleted & KPachonska & 3/17/20 2:15:00 PM \\
\hline$\nabla$. & & \\
\hline \multicolumn{3}{|l|}{$\Delta$} \\
\hline Page 2: [46] Deleted & KPachonska & 3/17/20 2:15:00 PM \\
\hline$\nabla$ & & \\
\hline \multicolumn{3}{|l|}{$\Delta$} \\
\hline Page 2: [46] Deleted & KPachonska & 3/17/20 2:15:00 PM \\
\hline$\nabla$ & & \\
\hline \multicolumn{3}{|l|}{$\Delta$} \\
\hline Page 2: [46] Deleted & KPachonska & 3/17/20 2:15:00 PM \\
\hline$\nabla$ & & \\
\hline \multicolumn{3}{|l|}{$\Delta$} \\
\hline Page 2: [46] Deleted & KPachonska & 3/17/20 2:15:00 PM \\
\hline$\nabla$. & & \\
\hline \multicolumn{3}{|r|}{ - } \\
\hline Page 2: [46] Deleted & KPachonska & 3/17/20 2:15:00 PM \\
\hline$\nabla$ & \multicolumn{2}{|r|}{ 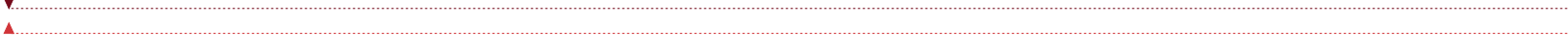 } \\
\hline Page 2: [46] Deleted & KPachonska & 3/17/20 2:15:00 PM \\
\hline$\nabla$ & & \\
\hline \multicolumn{3}{|l|}{$\nabla$} \\
\hline Page 2: [46] Deleted & KPachonska & 3/17/20 2:15:00 PM \\
\hline Page 2: [46] Deleted & KPachonska & 3/17/20 2:15:00 PM \\
\hline$\nabla$. & & \\
\hline \multicolumn{3}{|l|}{. } \\
\hline Page 2: [46] Deleted & KPachonska & 3/17/20 2:15:00 PM \\
\hline$\nabla$. & & \\
\hline \multicolumn{3}{|l|}{ 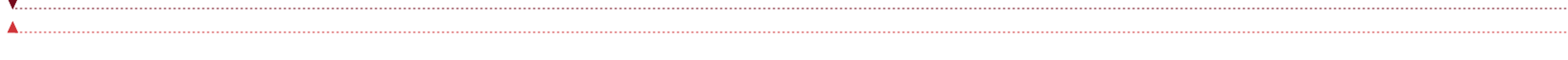 } \\
\hline Page 2: [46] Deleted & KPachonska & 3/17/20 2:15:00 PM \\
\hline$\nabla$. & & \\
\hline \multicolumn{3}{|r|}{ : } \\
\hline Page 2: [46] Deleted & KPachonska & 3/17/20 2:15:00 PM \\
\hline \multirow{2}{*}{\multicolumn{3}{|c|}{ 政. }} \\
\hline & & \\
\hline Page 2: [46] Deleted & KPachonska & 3/17/20 2:15:00 PM \\
\hline$\checkmark$ & & \\
\hline$\Delta$ & & \\
\hline Page 2: [46] Deleted & KPachonska & 3/17/20 2:15:00 PM \\
\hline
\end{tabular}


$\nabla$

$\checkmark$

$\nabla$

$\nabla$

$\nabla$

$\nabla$

Formatted

Page 2: [48] Formatted

KPachonska

3/17/20 2:15:00 PM

Formatted

Page 2: [48] Formatted

KPachonska

3/17/20 2:15:00 PM

Formatted

Page 2: [49] Formatted

KPachonska

3/17/20 2:15:00 PM

Formatted

Page 2: [50] Formatted

KPachonska

3/17/20 2:15:00 PM

Font: $12 \mathrm{pt}$

Page 2: [51] Formatted

Font: $12 \mathrm{pt}$

Page 2: [52] Formatted

KPachonska

3/17/20 2:15:00 PM

Font: $12 \mathrm{pt}$

Page 2: [53] Formatted

KPachonska

3/17/20 2:15:00 PM

Font: $12 \mathrm{pt}$

Page 2: [54] Formatted

KPachonska

3/17/20 2:15:00 PM

Font: $12 \mathrm{pt}$

Page 2: [55] Formatted

KPachonska

3/17/20 2:15:00 PM

Font: $12 \mathrm{pt}$ 


\begin{tabular}{|c|c|c|}
\hline Page 2: [56] Formatted & KPachonska & 3/17/20 2:15:00 PM \\
\hline \multicolumn{3}{|l|}{ Font: $12 \mathrm{pt}$} \\
\hline Page 2: [56] Formatted & KPachonska & 3/17/20 2:15:00 PM \\
\hline \multicolumn{3}{|l|}{ Font: $12 \mathrm{pt}$} \\
\hline Page 2: [57] Formatted & KPachonska & 3/17/20 2:15:00 PM \\
\hline \multicolumn{3}{|l|}{ Font: $12 \mathrm{pt}$} \\
\hline Page 2: [58] Formatted & KPachonska & 3/17/20 2:15:00 PM \\
\hline \multicolumn{3}{|l|}{ Font: $12 \mathrm{pt}$} \\
\hline Page 2: [59] Formatted & KPachonska & 3/17/20 2:15:00 PM \\
\hline \multicolumn{3}{|l|}{ Font: $12 \mathrm{pt}$} \\
\hline Page 2: [60] Formatted & KPachonska & 3/17/20 2:15:00 PM \\
\hline \multicolumn{3}{|l|}{ Font: $12 \mathrm{pt}$} \\
\hline Page 2: [61] Formatted & KPachonska & 3/17/20 2:15:00 PM \\
\hline \multicolumn{3}{|l|}{ Font: $12 \mathrm{pt}$} \\
\hline Page 2: [62] Formatted & KPachonska & 3/17/20 2:15:00 PM \\
\hline \multicolumn{3}{|l|}{ Font: $12 \mathrm{pt}$} \\
\hline Page 2: [63] Formatted & KPachonska & 3/17/20 2:15:00 PM \\
\hline \multicolumn{3}{|l|}{ Font: $12 \mathrm{pt}$} \\
\hline Page 2: [64] Formatted & KPachonska & 3/17/20 2:15:00 PM \\
\hline \multicolumn{3}{|l|}{ Font: $12 \mathrm{pt}$} \\
\hline Page 2: [64] Formatted & KPachonska & 3/17/20 2:15:00 PM \\
\hline \multicolumn{3}{|l|}{ Font: $12 \mathrm{pt}$} \\
\hline Page 2: [65] Formatted & KPachonska & 3/17/20 2:15:00 PM \\
\hline \multicolumn{3}{|l|}{ Font: $12 \mathrm{pt}$} \\
\hline Page 2: [66] Formatted & KPachonska & 3/17/20 2:15:00 PM \\
\hline \multicolumn{3}{|l|}{ Font: $12 \mathrm{pt}$} \\
\hline Page 2: [66] Formatted & KPachonska & 3/17/20 2:15:00 PM \\
\hline \multicolumn{3}{|l|}{ Font: $12 \mathrm{pt}$} \\
\hline Page 2: [67] Formatted & KPachonska & 3/17/20 2:15:00 PM \\
\hline \multicolumn{3}{|l|}{ Font: $12 \mathrm{pt}$} \\
\hline Page 2: [67] Formatted & KPachonska & 3/17/20 2:15:00 PM \\
\hline \multicolumn{3}{|l|}{ Font: $12 \mathrm{pt}$} \\
\hline Page 2: [68] Formatted & KPachonska & 3/17/20 2:15:00 PM \\
\hline \multicolumn{3}{|c|}{ Font: 12 pt, Font color: Custom Color(RGB(17,17,17)) } \\
\hline Page 2: [69] Deleted & KPachonska & 3/17/20 2:15:00 PM \\
\hline 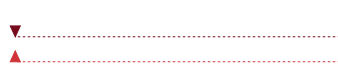 & & \\
\hline Page 2: $[70]$ Formatted & KPachonska & 3/17/20 2:15:00 PM \\
\hline \multicolumn{3}{|l|}{ Font: $12 \mathrm{pt}$} \\
\hline Page 2: [71] Formatted & KPachonska & 3/17/20 2:15:00 PM \\
\hline
\end{tabular}


Font: $12 \mathrm{pt}$

Page 2: [72] Formatted

KPachonska

3/17/20 2:15:00 PM

Font: $12 \mathrm{pt}$

Page 2: [73] Formatted

KPachonska

3/17/20 2:15:00 PM

Font: $12 \mathrm{pt}$

Page 2: [74] Formatted

KPachonska

3/17/20 2:15:00 PM

Font: $12 \mathrm{pt}$

Page 2: [75] Formatted

KPachonska

3/17/20 2:15:00 PM

Font: $12 \mathrm{pt}$

Page 2: [76] Formatted

KPachonska

3/17/20 2:15:00 PM

Font: $12 \mathrm{pt}$

Page 2: [77] Formatted

KPachonska

3/17/20 2:15:00 PM

Font: $12 \mathrm{pt}$

Page 2: [78] Formatted

KPachonska

3/17/20 2:15:00 PM

Font: $12 \mathrm{pt}$

Page 2: [79] Formatted

KPachonska

3/17/20 2:15:00 PM

Font: $12 \mathrm{pt}$

Page 2: [80] Formatted

KPachonska

3/17/20 2:15:00 PM

Font: $12 \mathrm{pt}$

Page 2: [81] Formatted

KPachonska

3/17/20 2:15:00 PM

Font: $12 \mathrm{pt}$

Page 2: [82] Formatted

KPachonska

3/17/20 2:15:00 PM

Font: $12 \mathrm{pt}$

Page 2: [83] Formatted

KPachonska

3/17/20 2:15:00 PM

Font: $12 \mathrm{pt}$

Page 2: [84] Formatted

KPachonska

3/17/20 2:15:00 PM

Font: $12 \mathrm{pt}$

Page 2: [85] Formatted

KPachonska

3/17/20 2:15:00 PM

Font: $12 \mathrm{pt}$

Page 2: [86] Formatted

KPachonska

3/17/20 2:15:00 PM

Font: $12 \mathrm{pt}$

Page 2: [87] Formatted

KPachonska

3/17/20 2:15:00 PM

Font: $12 \mathrm{pt}$

\begin{tabular}{|lll|}
\hline Page 2: $[88]$ Formatted & KPachonska & $3 / 17 / 20 ~ 2: 15: 00 ~ P M$ \\
\hline Font: $12 \mathrm{pt}$ & & \\
\hline Page 2: $[89]$ Formatted & KPachonska & $3 / 17 / 20$ 2:15:00 PM \\
\hline Font: $12 \mathrm{pt}$ & & $3 / 17 / 202: 15: 00$ PM \\
\hline Page 2: $[90]$ Formatted & KPachonska \\
\hline Font: $12 \mathrm{pt}$ & & $3 / 17 / 202: 15: 00$ PM \\
\hline Page 2: $[91]$ Formatted & KPachonska & \\
\hline
\end{tabular}


Font: $12 \mathrm{pt}$

Page 2: [92] Formatted

KPachonska

3/17/20 2:15:00 PM

Font: $12 \mathrm{pt}$

Page 2: [93] Formatted

KPachonska

3/17/20 2:15:00 PM

Font: $12 \mathrm{pt}$

Page 2: [94] Formatted

KPachonska

3/17/20 2:15:00 PM

Font: $12 \mathrm{pt}$

Page 2: [95] Formatted

KPachonska

3/17/20 2:15:00 PM

Font: $12 \mathrm{pt}$

Page 2: [96] Formatted

KPachonska

3/17/20 2:15:00 PM

Font: $12 \mathrm{pt}$

Page 2: [97] Formatted

KPachonska

3/17/20 2:15:00 PM

Font: $12 \mathrm{pt}$

Page 2: [98] Formatted

KPachonska

3/17/20 2:15:00 PM

Font: $12 \mathrm{pt}$

Page 2: [99] Formatted

KPachonska

3/17/20 2:15:00 PM

Font: $12 \mathrm{pt}$

Page 2: [100] Formatted

KPachonska

3/17/20 2:15:00 PM

Font: $12 \mathrm{pt}$

Page 2: [101] Formatted

KPachonska

3/17/20 2:15:00 PM

Font: $12 \mathrm{pt}$

Page 2: [102] Formatted

KPachonska

3/17/20 2:15:00 PM

Font: $12 \mathrm{pt}$

Page 2: [103] Formatted

KPachonska

3/17/20 2:15:00 PM

Font: $12 \mathrm{pt}$

Page 2: [104] Formatted

KPachonska

3/17/20 2:15:00 PM

Font: $12 \mathrm{pt}$

Page 2: [105] Deleted

KPachonska

3/17/20 2:15:00 PM

$\nabla$

Page 2: [106] Formatted

KPachonska

3/17/20 2:15:00 PM

Font: $12 \mathrm{pt}$

Page 2: [106] Formatted

KPachonska

3/17/20 2:15:00 PM

Font: $12 \mathrm{pt}$

Page 2: [107] Formatted

KPachonska

3/17/20 2:15:00 PM

Font: $12 \mathrm{pt}$

Page 2: [108] Formatted

KPachonska

3/17/20 2:15:00 PM

Font: $12 \mathrm{pt}$

Page 2: [109] Formatted

KPachonska

3/17/20 2:15:00 PM

Font: $12 \mathrm{pt}$ 


\begin{tabular}{|c|c|c|}
\hline Page 2: [110] Formatted & KPachonska & 3/17/20 2:15:00 PM \\
\hline \multicolumn{3}{|l|}{ Font: $12 \mathrm{pt}$} \\
\hline Page 2: [111] Formatted & KPachonska & 3/17/20 2:15:00 PM \\
\hline \multicolumn{3}{|l|}{ Font: $12 \mathrm{pt}$} \\
\hline Page 2: [112] Formatted & KPachonska & 3/17/20 2:15:00 PM \\
\hline \multicolumn{3}{|l|}{ Font: $12 \mathrm{pt}$} \\
\hline Page 2: [113] Formatted & KPachonska & 3/17/20 2:15:00 PM \\
\hline \multicolumn{3}{|l|}{ Font: $12 \mathrm{pt}$} \\
\hline Page 2: [114] Formatted & KPachonska & 3/17/20 2:15:00 PM \\
\hline \multicolumn{3}{|l|}{ Font: $12 \mathrm{pt}$} \\
\hline Page 2: [115] Formatted & KPachonska & 3/17/20 2:15:00 PM \\
\hline \multicolumn{3}{|l|}{ Font: $12 \mathrm{pt}$} \\
\hline Page 2: [115] Formatted & KPachonska & 3/17/20 2:15:00 PM \\
\hline \multicolumn{3}{|l|}{ Font: $12 \mathrm{pt}$} \\
\hline Page 2: [116] Formatted & KPachonska & 3/17/20 2:15:00 PM \\
\hline \multicolumn{3}{|l|}{ Font: $12 \mathrm{pt}$} \\
\hline Page 2: [117] Formatted & KPachonska & 3/17/20 2:15:00 PM \\
\hline \multicolumn{3}{|l|}{ Font: $12 \mathrm{pt}$} \\
\hline Page 2: [118] Formatted & KPachonska & 3/17/20 2:15:00 PM \\
\hline \multicolumn{3}{|l|}{ Font: $12 \mathrm{pt}$} \\
\hline Page 2: [119] Formatted & KPachonska & 3/17/20 2:15:00 PM \\
\hline \multicolumn{3}{|l|}{ Font: 14 pt, Bold } \\
\hline Page 2: [120] Formatted & KPachonska & 3/17/20 2:15:00 PM \\
\hline \multicolumn{3}{|c|}{ Normal, Space After: 8 pt, Line spacing: Multiple 1.15 li, Pattern: Clear (White) } \\
\hline Page 2: [121] Formatted & KPachonska & 3/17/20 2:15:00 PM \\
\hline \multicolumn{3}{|l|}{ Font: $12 \mathrm{pt}$} \\
\hline Page 2: [122] Formatted & KPachonska & 3/17/20 2:15:00 PM \\
\hline \multicolumn{3}{|l|}{ Font: $12 \mathrm{pt}$} \\
\hline Page 2: [122] Formatted & KPachonska & 3/17/20 2:15:00 PM \\
\hline \multicolumn{3}{|l|}{ Font: $12 \mathrm{pt}$} \\
\hline Page 2: [123] Formatted & KPachonska & 3/17/20 2:15:00 PM \\
\hline \multicolumn{3}{|l|}{ Font: $12 \mathrm{pt}$} \\
\hline Page 2: [124] Formatted & KPachonska & 3/17/20 2:15:00 PM \\
\hline \multicolumn{3}{|l|}{ Font: $12 \mathrm{pt}$} \\
\hline Page 2: [125] Formatted & KPachonska & 3/17/20 2:15:00 PM \\
\hline \multicolumn{3}{|l|}{ Font: $12 \mathrm{pt}$} \\
\hline Page 2: [126] Deleted & KPachonska & 3/17/20 2:15:00 PM \\
\hline$\nabla$ & & \\
\hline Page 1: [127] Formatted & KPachonska & 3/17/20 2:15:00 PM \\
\hline
\end{tabular}


Normal, Tab stops: 6.26", Right

Page 1: [128] Formatted

KPachonska

3/17/20 2:15:00 PM

Normal, Tab stops: 6.26", Right

Page 3: [129] Formatted KPachonska

3/17/20 2:15:00 PM

Font: $12 \mathrm{pt}$

Page 3: [130] Formatted

KPachonska

3/17/20 2:15:00 PM

Font: $12 \mathrm{pt}$

Page 3: [131] Formatted

KPachonska

3/17/20 2:15:00 PM

Font: $12 \mathrm{pt}$

Page 3: [131] Formatted

KPachonska

3/17/20 2:15:00 PM

Font: $12 \mathrm{pt}$

Page 3: [132] Formatted

KPachonska

3/17/20 2:15:00 PM

Font: $12 \mathrm{pt}$

Page 3: [133] Formatted

KPachonska

3/17/20 2:15:00 PM

Font: $12 \mathrm{pt}$

Page 3: [134] Formatted

KPachonska

3/17/20 2:15:00 PM

Font: $12 \mathrm{pt}$

Page 3: [135] Formatted

KPachonska

3/17/20 2:15:00 PM

Font: $12 \mathrm{pt}$

Page 3: [136] Formatted

KPachonska

3/17/20 2:15:00 PM

Font: $12 \mathrm{pt}$

Page 3: [137] Formatted

KPachonska

3/17/20 2:15:00 PM

Font: $12 \mathrm{pt}$

Page 3: [138] Formatted

KPachonska

3/17/20 2:15:00 PM

Font: $12 \mathrm{pt}$

Page 3: [139] Formatted

KPachonska

3/17/20 2:15:00 PM

Font: $12 \mathrm{pt}$

Page 3: [140] Formatted

KPachonska

3/17/20 2:15:00 PM

Font: $12 \mathrm{pt}$

Page 3: [141] Formatted

KPachonska

3/17/20 2:15:00 PM

Font: $12 \mathrm{pt}$

Page 3: [142] Formatted

KPachonska

3/17/20 2:15:00 PM

Font: $12 \mathrm{pt}$

\begin{tabular}{|c|c|c|}
\hline Page 3: [143] Formatted & KPachonska & 3/17/20 2:15:00 PM \\
\hline \multicolumn{3}{|l|}{ Font: $12 \mathrm{pt}$} \\
\hline Page 3: [144] Formatted & KPachonska & 3/17/20 2:15:00 PM \\
\hline \multicolumn{3}{|l|}{ Font: $12 \mathrm{pt}$} \\
\hline Page 3: [145] Formatted & KPachonska & 3/17/20 2:15:00 PM \\
\hline \multicolumn{3}{|l|}{ Font: $12 \mathrm{pt}$} \\
\hline Page 3: [146] Formatted & KPachonska & 3/17/20 2:15:00 PM \\
\hline
\end{tabular}


Font: $12 \mathrm{pt}$

Page 3: [147] Formatted

KPachonska

3/17/20 2:15:00 PM

Font: $12 \mathrm{pt}$

Page 3: [148] Formatted

KPachonska

3/17/20 2:15:00 PM

Font: $12 \mathrm{pt}$

Page 3: [149] Formatted

KPachonska

3/17/20 2:15:00 PM

Font: $12 \mathrm{pt}$

Page 3: [150] Formatted

KPachonska

3/17/20 2:15:00 PM

Font: $12 \mathrm{pt}$

Page 3: [151] Formatted

KPachonska

3/17/20 2:15:00 PM

Font: $12 \mathrm{pt}$

Page 3: [152] Formatted

KPachonska

3/17/20 2:15:00 PM

Font: $12 \mathrm{pt}$

Page 3: [153] Formatted

KPachonska

3/17/20 2:15:00 PM

Font: $12 \mathrm{pt}$

Page 3: [154] Formatted

KPachonska

3/17/20 2:15:00 PM

Font: $12 \mathrm{pt}$

Page 3: [155] Formatted

KPachonska

3/17/20 2:15:00 PM

Font: $12 \mathrm{pt}$

Page 3: [156] Formatted

KPachonska

3/17/20 2:15:00 PM

Font: $12 \mathrm{pt}$

Page 3: [157] Formatted

KPachonska

3/17/20 2:15:00 PM

Font: $12 \mathrm{pt}$

Page 3: [158] Formatted

KPachonska

3/17/20 2:15:00 PM

Font: $12 \mathrm{pt}$

Page 3: [159] Formatted

KPachonska

3/17/20 2:15:00 PM

Font: $12 \mathrm{pt}$

Page 3: [160] Formatted

KPachonska

3/17/20 2:15:00 PM

Font: $12 \mathrm{pt}$

Page 3: [161] Formatted

KPachonska

3/17/20 2:15:00 PM

Font: $12 \mathrm{pt}$

Page 3: [162] Formatted

KPachonska

3/17/20 2:15:00 PM

Font: $12 \mathrm{pt}$

\begin{tabular}{|c|c|c|}
\hline Page 3: [163] Formatted & KPachonska & 3/17/20 2:15:00 PM \\
\hline \multicolumn{3}{|c|}{ Normal, Space After: 8 pt, Line spacing: Multiple 1.15 li, Pattern: Clear (White) } \\
\hline Page 3: [164] Formatted & KPachonska & 3/17/20 2:15:00 PM \\
\hline \multicolumn{3}{|l|}{ Font: $12 \mathrm{pt}$} \\
\hline Page 3: [165] Formatted & KPachonska & 3/17/20 2:15:00 PM \\
\hline \multicolumn{3}{|l|}{ Font: $12 \mathrm{pt}$} \\
\hline Page 3: [166] Formatted & KPachonska & 3/17/20 2:15:00 PM \\
\hline
\end{tabular}


Font: $12 \mathrm{pt}$

Page 3: [167] Formatted

KPachonska

3/17/20 2:15:00 PM

Font: $12 \mathrm{pt}$

Page 3: [168] Formatted

KPachonska

3/17/20 2:15:00 PM

Font: $12 \mathrm{pt}$

Page 3: [169] Formatted

KPachonska

3/17/20 2:15:00 PM

Font: $12 \mathrm{pt}$

Page 3: [170] Formatted

KPachonska

3/17/20 2:15:00 PM

Font: $12 \mathrm{pt}$

Page 3: [171] Formatted

KPachonska

3/17/20 2:15:00 PM

Font: $12 \mathrm{pt}$

Page 3: [172] Formatted

KPachonska

3/17/20 2:15:00 PM

Font: $12 \mathrm{pt}$

Page 3: [173] Formatted

KPachonska

3/17/20 2:15:00 PM

Font: $12 \mathrm{pt}$

Page 3: [174] Formatted

KPachonska

3/17/20 2:15:00 PM

Font: $12 \mathrm{pt}$

Page 3: [175] Formatted

KPachonska

3/17/20 2:15:00 PM

Font: $12 \mathrm{pt}$

Page 3: [176] Formatted

KPachonska

3/17/20 2:15:00 PM

Font: $12 \mathrm{pt}$

Page 3: [177] Formatted

KPachonska

3/17/20 2:15:00 PM

Font: $12 \mathrm{pt}$

Page 3: [178] Formatted

KPachonska

3/17/20 2:15:00 PM

Font: $12 \mathrm{pt}$

Page 3: [179] Formatted

KPachonska

3/17/20 2:15:00 PM

Font: $12 \mathrm{pt}$

Page 3: [180] Formatted

KPachonska

3/17/20 2:15:00 PM

Font: $12 \mathrm{pt}$

Page 3: [181] Formatted

KPachonska

3/17/20 2:15:00 PM

Font: $12 \mathrm{pt}$

Page 3: [182] Formatted

KPachonska

3/17/20 2:15:00 PM

Font: $12 \mathrm{pt}$

\begin{tabular}{|c|c|c|}
\hline Page 3: [183] Formatted & KPachonska & 3/17/20 2:15:00 PM \\
\hline \multicolumn{3}{|l|}{ Font: $12 \mathrm{pt}$} \\
\hline Page 3: [184] Formatted & KPachonska & 3/17/20 2:15:00 PM \\
\hline \multicolumn{3}{|l|}{ Font: $12 \mathrm{pt}$} \\
\hline Page 3: [185] Formatted & KPachonska & 3/17/20 2:15:00 PM \\
\hline \multicolumn{3}{|l|}{ Font: $12 \mathrm{pt}$} \\
\hline Page 3: [186] Formatted & KPachonska & 3/17/20 2:15:00 PM \\
\hline
\end{tabular}


Font: $12 \mathrm{pt}$

Page 3: [187] Formatted

KPachonska

3/17/20 2:15:00 PM

Font: $12 \mathrm{pt}$

Page 3: [188] Formatted

KPachonska

3/17/20 2:15:00 PM

Font: $12 \mathrm{pt}$

Page 3: [188] Formatted

KPachonska

3/17/20 2:15:00 PM

Font: $12 \mathrm{pt}$

Page 3: [189] Formatted

KPachonska

3/17/20 2:15:00 PM

Font: $12 \mathrm{pt}$

Page 3: [190] Formatted

KPachonska

3/17/20 2:15:00 PM

Font: $12 \mathrm{pt}$

Page 3: [191] Formatted

KPachonska

3/17/20 2:15:00 PM

Font: $12 \mathrm{pt}$

Page 3: [192] Formatted

KPachonska

3/17/20 2:15:00 PM

Font: $12 \mathrm{pt}$

Page 3: [193] Formatted

KPachonska

3/17/20 2:15:00 PM

Font: $12 \mathrm{pt}$

Page 3: [194] Formatted

KPachonska

3/17/20 2:15:00 PM

Font: $12 \mathrm{pt}$

Page 3: [195] Formatted

KPachonska

3/17/20 2:15:00 PM

Font: $12 \mathrm{pt}$

Page 3: [196] Formatted

KPachonska

3/17/20 2:15:00 PM

Font: $12 \mathrm{pt}$

Page 3: [197] Formatted

KPachonska

3/17/20 2:15:00 PM

Font: $12 \mathrm{pt}$

Page 3: [198] Formatted

KPachonska

3/17/20 2:15:00 PM

Font: $12 \mathrm{pt}$

Page 3: [199] Formatted

KPachonska

3/17/20 2:15:00 PM

Font: $12 \mathrm{pt}$

Page 3: [200] Formatted

KPachonska

3/17/20 2:15:00 PM

Font: $12 \mathrm{pt}$

Page 3: [201] Formatted

KPachonska

3/17/20 2:15:00 PM

Font: $12 \mathrm{pt}$

\begin{tabular}{|llr|}
\hline Page 3: $[202]$ Formatted & KPachonska & $3 / 17 / 20 ~ 2: 15: 00 ~ P M$ \\
\hline Font: $12 \mathrm{pt}$ & & \\
\hline Page 3: $[203]$ Formatted & KPachonska & $3 / 17 / 20$ 2:15:00 PM \\
\hline Font: $12 \mathrm{pt}$ & & $3 / 17 / 202: 15: 00$ PM \\
\hline Page 3: $[204]$ Formatted & KPachonska \\
\hline Font: $12 \mathrm{pt}$ & & $3 / 17 / 20$ 2:15:00 PM \\
\hline Page 3: $[204]$ Formatted & KPachonska & \\
\hline
\end{tabular}


Font: $12 \mathrm{pt}$

Page 3: [205] Formatted

KPachonska

3/17/20 2:15:00 PM

Font: $12 \mathrm{pt}$

Page 3: [206] Formatted

KPachonska

3/17/20 2:15:00 PM

Font: $12 \mathrm{pt}$

Page 3: [207] Deleted

KPachonska

3/17/20 2:15:00 PM

Page 3: [208] Formatted

KPachonska

3/17/20 2:15:00 PM

Font: $12 \mathrm{pt}$

Page 3: [209] Formatted

KPachonska

3/17/20 2:15:00 PM

Font: $12 \mathrm{pt}$

Page 3: [210] Formatted

KPachonska

3/17/20 2:15:00 PM

Font: $12 \mathrm{pt}$

\begin{tabular}{|c|c|c|}
\hline Page 3: [211] Formatted & KPachonska & 3/17/20 2:15:00 PM \\
\hline \multicolumn{3}{|l|}{ Font: $12 \mathrm{pt}$} \\
\hline Page 3: [212] Formatted & KPachonska & 3/17/20 2:15:00 PM \\
\hline \multicolumn{3}{|l|}{ Font: $12 \mathrm{pt}$} \\
\hline Page 3: [213] Formatted & KPachonska & 3/17/20 2:15:00 PM \\
\hline \multicolumn{3}{|l|}{ Font: $12 \mathrm{pt}$} \\
\hline Page 3: [214] Formatted & KPachonska & 3/17/20 2:15:00 PM \\
\hline \multicolumn{3}{|l|}{ Font: $12 \mathrm{pt}$} \\
\hline Page 3: [215] Formatted & KPachonska & 3/17/20 2:15:00 PM \\
\hline \multicolumn{3}{|l|}{ Font: $12 \mathrm{pt}$} \\
\hline Page 3: [216] Formatted & KPachonska & 3/17/20 2:15:00 PM \\
\hline \multicolumn{3}{|l|}{ Font: $12 \mathrm{pt}$} \\
\hline Page 3: [217] Formatted & KPachonska & 3/17/20 2:15:00 PM \\
\hline \multicolumn{3}{|l|}{ Font: $12 \mathrm{pt}$} \\
\hline Page 3: [218] Formatted & KPachonska & 3/17/20 2:15:00 PM \\
\hline \multicolumn{3}{|l|}{ Font: $12 \mathrm{pt}$} \\
\hline Page 3: [219] Formatted & KPachonska & 3/17/20 2:15:00 PM \\
\hline \multicolumn{3}{|l|}{ Font: $12 \mathrm{pt}$} \\
\hline Page 3: $[220]$ Formatted & KPachonska & 3/17/20 2:15:00 PM \\
\hline \multicolumn{3}{|l|}{ Font: $12 \mathrm{pt}$} \\
\hline Page 3: [221] Formatted & KPachonska & 3/17/20 2:15:00 PM \\
\hline \multicolumn{3}{|l|}{ Font: $12 \mathrm{pt}$} \\
\hline Page 3: [222] Formatted & KPachonska & 3/17/20 2:15:00 PM \\
\hline \multicolumn{3}{|l|}{ Font: $12 \mathrm{pt}$} \\
\hline Page 3: [223] Formatted & KPachonska & 3/17/20 2:15:00 PM \\
\hline
\end{tabular}

Font: $12 \mathrm{pt}$ 


\begin{tabular}{|c|c|c|}
\hline Page 3: $[224]$ Formatted & KPachonska & 3/17/20 2:15:00 PM \\
\hline \multicolumn{3}{|l|}{ Font: $12 \mathrm{pt}$} \\
\hline Page 3: [225] Formatted & KPachonska & 3/17/20 2:15:00 PM \\
\hline \multicolumn{3}{|l|}{ Font: $12 \mathrm{pt}$} \\
\hline Page 3: [226] Formatted & KPachonska & 3/17/20 2:15:00 PM \\
\hline \multicolumn{3}{|l|}{ Font: $12 \mathrm{pt}$} \\
\hline Page 3: [227] Formatted & KPachonska & 3/17/20 2:15:00 PM \\
\hline \multicolumn{3}{|l|}{ Font: $12 \mathrm{pt}$} \\
\hline Page 3: [228] Formatted & KPachonska & 3/17/20 2:15:00 PM \\
\hline \multicolumn{3}{|l|}{ Font: $12 \mathrm{pt}$} \\
\hline Page 3: [229] Formatted & KPachonska & 3/17/20 2:15:00 PM \\
\hline \multicolumn{3}{|l|}{ Font: $12 \mathrm{pt}$} \\
\hline Page 3: [229] Formatted & KPachonska & 3/17/20 2:15:00 PM \\
\hline \multicolumn{3}{|l|}{ Font: $12 \mathrm{pt}$} \\
\hline Page 3: $[230]$ Formatted & KPachonska & 3/17/20 2:15:00 PM \\
\hline \multicolumn{3}{|l|}{ Font: $12 \mathrm{pt}$} \\
\hline Page 3: [231] Formatted & KPachonska & 3/17/20 2:15:00 PM \\
\hline \multicolumn{3}{|l|}{ Font: $12 \mathrm{pt}$} \\
\hline Page 3: [232] Formatted & KPachonska & 3/17/20 2:15:00 PM \\
\hline \multicolumn{3}{|l|}{ Font: $12 \mathrm{pt}$} \\
\hline Page 3: [232] Formatted & KPachonska & 3/17/20 2:15:00 PM \\
\hline \multicolumn{3}{|l|}{ Font: $12 \mathrm{pt}$} \\
\hline Page 3: [233] Formatted & KPachonska & 3/17/20 2:15:00 PM \\
\hline \multicolumn{3}{|l|}{ Font: $12 \mathrm{pt}$} \\
\hline Page 3: [234] Formatted & KPachonska & 3/17/20 2:15:00 PM \\
\hline \multicolumn{3}{|l|}{ Font: $12 \mathrm{pt}$} \\
\hline Page 3: [235] Formatted & KPachonska & 3/17/20 2:15:00 PM \\
\hline \multicolumn{3}{|l|}{ Font: $12 \mathrm{pt}$} \\
\hline Page 3: [236] Formatted & KPachonska & 3/17/20 2:15:00 PM \\
\hline \multicolumn{3}{|l|}{ Font: $12 \mathrm{pt}$} \\
\hline Page 3: [237] Deleted & KPachonska & 3/17/20 2:15:00 PM \\
\hline$\nabla$ & & \\
\hline Page 3: [238] Formatted & KPachonska & 3/17/20 2:15:00 PM \\
\hline \multicolumn{3}{|l|}{ Font: $12 \mathrm{pt}$} \\
\hline Page 3: [239] Formatted & KPachonska & 3/17/20 2:15:00 PM \\
\hline \multicolumn{3}{|l|}{ Font: $12 \mathrm{pt}$} \\
\hline Page 3: [239] Formatted & KPachonska & 3/17/20 2:15:00 PM \\
\hline \multicolumn{3}{|l|}{ Font: $12 \mathrm{pt}$} \\
\hline Page 3: [240] Formatted & KPachonska & 3/17/20 2:15:00 PM \\
\hline
\end{tabular}


Font: $12 \mathrm{pt}$

Page 3: [241] Formatted

KPachonska

3/17/20 2:15:00 PM

Font: $12 \mathrm{pt}$

Page 3: [242] Formatted

KPachonska

3/17/20 2:15:00 PM

Font: $12 \mathrm{pt}$

Page 3: [243] Formatted

KPachonska

3/17/20 2:15:00 PM

Font: $12 \mathrm{pt}$

Page 3: [244] Formatted

KPachonska

3/17/20 2:15:00 PM

Font: $12 \mathrm{pt}$

Page 3: [245] Formatted

KPachonska

3/17/20 2:15:00 PM

Font: $12 \mathrm{pt}$

Page 3: [246] Formatted

KPachonska

3/17/20 2:15:00 PM

Font: $12 \mathrm{pt}$

Page 3: [247] Formatted

KPachonska

3/17/20 2:15:00 PM

Font: $12 \mathrm{pt}$

Page 3: [248] Formatted

KPachonska

3/17/20 2:15:00 PM

Font: $12 \mathrm{pt}$

Page 3: [249] Formatted

KPachonska

3/17/20 2:15:00 PM

Font: $12 \mathrm{pt}$

Page 3: [250] Formatted

KPachonska

3/17/20 2:15:00 PM

Font: $12 \mathrm{pt}$

Page 3: [251] Formatted

KPachonska

3/17/20 2:15:00 PM

Font: $12 \mathrm{pt}$

Page 3: [251] Formatted

KPachonska

3/17/20 2:15:00 PM

Font: $12 \mathrm{pt}$

Page 3: [252] Formatted

KPachonska

3/17/20 2:15:00 PM

Font: $12 \mathrm{pt}$

Page 3: [252] Formatted

KPachonska

3/17/20 2:15:00 PM

Font: $12 \mathrm{pt}$

Page 1: [253] Formatted

KPachonska

3/17/20 2:15:00 PM

Normal, Tab stops: 6.26", Right

Page 1: [254] Formatted

KPachonska

3/17/20 2:15:00 PM

Normal, Tab stops: 6.26", Right

Page 4: [255] Formatted

KPachonska

3/17/20 2:15:00 PM

Font: $12 \mathrm{pt}$

Page 4: [255] Formatted

KPachonska

3/17/20 2:15:00 PM

Font: $12 \mathrm{pt}$

Page 4: [256] Formatted

KPachonska

3/17/20 2:15:00 PM

Font: $12 \mathrm{pt}$

Page 4: [256] Formatted

KPachonska

3/17/20 2:15:00 PM 
Font: $12 \mathrm{pt}$

Page 4: [257] Formatted

KPachonska

3/17/20 2:15:00 PM

Font: $12 \mathrm{pt}$

Page 4: [258] Formatted

KPachonska

3/17/20 2:15:00 PM

Font: $12 \mathrm{pt}$

Page 4: [259] Formatted

KPachonska

3/17/20 2:15:00 PM

Font: $12 \mathrm{pt}$

Page 4: [259] Formatted

KPachonska

3/17/20 2:15:00 PM

Font: $12 \mathrm{pt}$

Page 4: [260] Formatted

KPachonska

3/17/20 2:15:00 PM

Font: $12 \mathrm{pt}$

Page 4: [261] Formatted

KPachonska

3/17/20 2:15:00 PM

Font: $12 \mathrm{pt}$

Page 4: [262] Formatted

KPachonska

3/17/20 2:15:00 PM

Font: $12 \mathrm{pt}$

Page 4: [262] Formatted

KPachonska

3/17/20 2:15:00 PM

Font: $12 \mathrm{pt}$

Page 4: [263] Formatted

KPachonska

3/17/20 2:15:00 PM

Font: $12 \mathrm{pt}$

Page 4: [263] Formatted

KPachonska

3/17/20 2:15:00 PM

Font: $12 \mathrm{pt}$

Page 4: [264] Formatted

KPachonska

3/17/20 2:15:00 PM

Font: $12 \mathrm{pt}$

Page 4: [265] Formatted

KPachonska

3/17/20 2:15:00 PM

Font: $12 \mathrm{pt}$

Page 4: [266] Formatted

KPachonska

3/17/20 2:15:00 PM

Font: $12 \mathrm{pt}$

Page 4: [267] Formatted

KPachonska

3/17/20 2:15:00 PM

Font: $12 \mathrm{pt}$

Page 4: [267] Formatted

KPachonska

3/17/20 2:15:00 PM

Font: $12 \mathrm{pt}$

Page 4: [268] Formatted

KPachonska

3/17/20 2:15:00 PM

Font: $12 \mathrm{pt}$

\begin{tabular}{|c|c|c|}
\hline Page 4: [269] Formatted & KPachonska & 3/17/20 2:15:00 PM \\
\hline \multicolumn{3}{|l|}{ Font: $12 \mathrm{pt}$} \\
\hline Page 4: [270] Formatted & KPachonska & 3/17/20 2:15:00 PM \\
\hline \multicolumn{3}{|l|}{ Font: $12 \mathrm{pt}$} \\
\hline Page 4: [271] Formatted & KPachonska & 3/17/20 2:15:00 PM \\
\hline \multicolumn{3}{|l|}{ Font: $12 \mathrm{pt}$} \\
\hline Page 4: [272] Formatted & KPachonska & 3/17/20 2:15:00 PM \\
\hline
\end{tabular}


Font: $12 \mathrm{pt}$

Page 4: [273] Formatted

KPachonska

3/17/20 2:15:00 PM

Font: $12 \mathrm{pt}$

Page 4: [274] Formatted

KPachonska

3/17/20 2:15:00 PM

Font: $12 \mathrm{pt}$

Page 4: [275] Formatted

KPachonska

3/17/20 2:15:00 PM

Font: $12 \mathrm{pt}$

Page 4: [276] Formatted

KPachonska

3/17/20 2:15:00 PM

Font: $12 \mathrm{pt}$

Page 4: [277] Formatted

KPachonska

3/17/20 2:15:00 PM

Font: $12 \mathrm{pt}$

Page 4: [278] Formatted

KPachonska

3/17/20 2:15:00 PM

Font: $12 \mathrm{pt}$

Page 4: [279] Formatted

KPachonska

3/17/20 2:15:00 PM

Font: $12 \mathrm{pt}$

Page 4: [280] Formatted

KPachonska

3/17/20 2:15:00 PM

Font: $12 \mathrm{pt}$

Page 4: [281] Formatted

KPachonska

3/17/20 2:15:00 PM

Font: $12 \mathrm{pt}$

Page 4: [282] Formatted

KPachonska

3/17/20 2:15:00 PM

Font: $12 \mathrm{pt}$

Page 4: [283] Formatted

KPachonska

3/17/20 2:15:00 PM

Font: $12 \mathrm{pt}$

Page 4: [284] Formatted

KPachonska

3/17/20 2:15:00 PM

Font: $12 \mathrm{pt}$

Page 4: [285] Formatted

KPachonska

3/17/20 2:15:00 PM

Font: $12 \mathrm{pt}$

Page 4: [286] Formatted

KPachonska

3/17/20 2:15:00 PM

Font: $12 \mathrm{pt}$

Page 4: [286] Formatted

KPachonska

3/17/20 2:15:00 PM

Font: $12 \mathrm{pt}$

Page 4: [287] Formatted

KPachonska

3/17/20 2:15:00 PM

Font: $12 \mathrm{pt}$

\begin{tabular}{|c|c|c|}
\hline Page 4: [288] Formatted & KPachonska & 3/17/20 2:15:00 PM \\
\hline \multicolumn{3}{|l|}{ Font: $12 \mathrm{pt}$} \\
\hline Page 4: [289] Formatted & KPachonska & 3/17/20 2:15:00 PM \\
\hline \multicolumn{3}{|l|}{ Font: $12 \mathrm{pt}$} \\
\hline Page 4: [290] Formatted & KPachonska & 3/17/20 2:15:00 PM \\
\hline \multicolumn{3}{|l|}{ Font: $12 \mathrm{pt}$} \\
\hline Page 4: [290] Formatted & KPachonska & 3/17/20 2:15:00 PM \\
\hline
\end{tabular}


Font: $12 \mathrm{pt}$

Page 4: [291] Formatted

KPachonska

3/17/20 2:15:00 PM

Font: $12 \mathrm{pt}$

Page 4: [292] Formatted

KPachonska

3/17/20 2:15:00 PM

Font: $12 \mathrm{pt}$

Page 4: [293] Formatted

KPachonska

3/17/20 2:15:00 PM

Font: $12 \mathrm{pt}$

Page 4: [294] Formatted

KPachonska

3/17/20 2:15:00 PM

Font: $12 \mathrm{pt}$

Page 4: [295] Formatted

KPachonska

3/17/20 2:15:00 PM

Font: $12 \mathrm{pt}$

Page 4: [296] Formatted

KPachonska

3/17/20 2:15:00 PM

Font: $12 \mathrm{pt}$

Page 4: [297] Formatted

KPachonska

3/17/20 2:15:00 PM

Font: $12 \mathrm{pt}$

Page 4: [297] Formatted

KPachonska

3/17/20 2:15:00 PM

Font: $12 \mathrm{pt}$

Page 4: [298] Formatted

KPachonska

3/17/20 2:15:00 PM

Font: $12 \mathrm{pt}$

Page 4: [299] Formatted

KPachonska

3/17/20 2:15:00 PM

Font: $12 \mathrm{pt}$

Page 4: [300] Formatted

KPachonska

3/17/20 2:15:00 PM

Font: $12 \mathrm{pt}$

Page 4: [301] Formatted

KPachonska

3/17/20 2:15:00 PM

Font: $12 \mathrm{pt}$

Page 4: [302] Formatted

KPachonska

3/17/20 2:15:00 PM

Font: $12 \mathrm{pt}$

Page 4: [303] Formatted

KPachonska

3/17/20 2:15:00 PM

Font: $12 \mathrm{pt}$

Page 4: [304] Formatted

KPachonska

3/17/20 2:15:00 PM

Font: $12 \mathrm{pt}$

Page 4: [305] Formatted

KPachonska

3/17/20 2:15:00 PM

Font: $12 \mathrm{pt}$

\begin{tabular}{|lcc|}
\hline Page 4: $[306]$ Formatted & KPachonska & $3 / 17 / 20$ 2:15:00 PM \\
\hline Font: $12 \mathrm{pt}$ & & $3 / 17 / 20$ 2:15:00 PM \\
\hline Page 4: $[307]$ Formatted & KPachonska & \\
\hline Font: $12 \mathrm{pt}$ & & $3 / 17 / 202: 15: 00$ PM \\
\hline Page 4: $[308]$ Formatted & KPachonska \\
\hline Font: $12 \mathrm{pt}$ & & $3 / 17 / 202: 15: 00$ PM \\
\hline Page 4: $[309]$ Formatted & KPachonska & \\
\hline
\end{tabular}


Font: $12 \mathrm{pt}$

Page 4: [310] Formatted

KPachonska

3/17/20 2:15:00 PM

Font: $12 \mathrm{pt}$

Page 4: [311] Formatted

KPachonska

3/17/20 2:15:00 PM

Font: $12 \mathrm{pt}$

Page 4: [312] Formatted

KPachonska

3/17/20 2:15:00 PM

Font: $12 \mathrm{pt}$

Page 4: [313] Formatted

KPachonska

3/17/20 2:15:00 PM

Font: $12 \mathrm{pt}$

Page 4: [314] Formatted

KPachonska

3/17/20 2:15:00 PM

Font: $12 \mathrm{pt}$

Page 4: [315] Formatted

KPachonska

3/17/20 2:15:00 PM

Font: $12 \mathrm{pt}$

Page 4: [316] Formatted

KPachonska

3/17/20 2:15:00 PM

Font: $12 \mathrm{pt}$

Page 4: [317] Formatted

KPachonska

3/17/20 2:15:00 PM

Font: $12 \mathrm{pt}$

Page 4: [318] Formatted

KPachonska

3/17/20 2:15:00 PM

Font: $12 \mathrm{pt}$

Page 4: [319] Formatted

KPachonska

3/17/20 2:15:00 PM

Font: $12 \mathrm{pt}$

Page 4: [320] Formatted

KPachonska

3/17/20 2:15:00 PM

Font: $12 \mathrm{pt}$

Page 4: [321] Formatted

KPachonska

3/17/20 2:15:00 PM

Font: $12 \mathrm{pt}$

Page 4: [322] Formatted

KPachonska

3/17/20 2:15:00 PM

Font: $12 \mathrm{pt}$

Page 4: [323] Formatted

KPachonska

3/17/20 2:15:00 PM

Font: $12 \mathrm{pt}$

Page 4: [324] Formatted

KPachonska

3/17/20 2:15:00 PM

Font: $12 \mathrm{pt}$

Page 4: [325] Formatted

KPachonska

3/17/20 2:15:00 PM

Font: $12 \mathrm{pt}$

\begin{tabular}{|c|c|c|}
\hline Page 4: [326] Formatted & KPachonska & 3/17/20 2:15:00 PM \\
\hline \multicolumn{3}{|l|}{ Font: $12 \mathrm{pt}$} \\
\hline Page 4: [327] Formatted & KPachonska & 3/17/20 2:15:00 PM \\
\hline \multicolumn{3}{|l|}{ Font: $12 \mathrm{pt}$} \\
\hline Page 4: [328] Formatted & KPachonska & 3/17/20 2:15:00 PM \\
\hline \multicolumn{3}{|c|}{ Normal, Space After: 8 pt, Line spacing: Multiple 1.15 li, Pattern: Clear (White) } \\
\hline Page 4: [329] Formatted & KPachonska & 3/17/20 2:15:00 PM \\
\hline
\end{tabular}


Font: $12 \mathrm{pt}$

Page 4: [330] Formatted

KPachonska

3/17/20 2:15:00 PM

Font: $12 \mathrm{pt}$

Page 4: [331] Formatted

KPachonska

3/17/20 2:15:00 PM

Font: $12 \mathrm{pt}$

Page 4: [332] Formatted

KPachonska

3/17/20 2:15:00 PM

Font: $12 \mathrm{pt}$

Page 4: [333] Formatted

KPachonska

3/17/20 2:15:00 PM

Font: $12 \mathrm{pt}$

Page 4: [334] Formatted

KPachonska

3/17/20 2:15:00 PM

Font: $12 \mathrm{pt}$

Page 4: [335] Formatted

KPachonska

3/17/20 2:15:00 PM

Font: $12 \mathrm{pt}$

Page 4: [336] Formatted

KPachonska

3/17/20 2:15:00 PM

Font: $12 \mathrm{pt}$

Page 4: [337] Formatted

KPachonska

3/17/20 2:15:00 PM

Font: $12 \mathrm{pt}$

Page 4: [338] Formatted

KPachonska

3/17/20 2:15:00 PM

Font: $12 \mathrm{pt}$

Page 4: [339] Formatted

KPachonska

3/17/20 2:15:00 PM

Font: $12 \mathrm{pt}$

Page 4: [340] Formatted

KPachonska

3/17/20 2:15:00 PM

Font: $12 \mathrm{pt}$

Page 4: [341] Formatted

KPachonska

3/17/20 2:15:00 PM

Font: $12 \mathrm{pt}$

Page 4: [342] Formatted

KPachonska

3/17/20 2:15:00 PM

Font: $12 \mathrm{pt}$

Page 4: [343] Formatted

KPachonska

3/17/20 2:15:00 PM

Font: $12 \mathrm{pt}$

Page 4: [344] Formatted

KPachonska

3/17/20 2:15:00 PM

Font: $12 \mathrm{pt}$

Page 4: [345] Formatted

KPachonska

3/17/20 2:15:00 PM

Font: $12 \mathrm{pt}$

\begin{tabular}{|c|c|c|}
\hline Page 4: [346] Formatted & KPachonska & 3/17/20 2:15:00 PM \\
\hline \multicolumn{3}{|l|}{ Font: $12 \mathrm{pt}$} \\
\hline Page 4: [347] Formatted & KPachonska & 3/17/20 2:15:00 PM \\
\hline \multicolumn{3}{|l|}{ Font: $12 \mathrm{pt}$} \\
\hline Page 4: [348] Formatted & KPachonska & 3/17/20 2:15:00 PM \\
\hline \multicolumn{3}{|l|}{ Font: $12 \mathrm{pt}$} \\
\hline Page 4: [349] Formatted & KPachonska & 3/17/20 2:15:00 PM \\
\hline
\end{tabular}


Font: $12 \mathrm{pt}$

Page 4: [350] Formatted

KPachonska

3/17/20 2:15:00 PM

Font: $12 \mathrm{pt}$

Page 4: [351] Formatted

KPachonska

3/17/20 2:15:00 PM

Font: $12 \mathrm{pt}$

\begin{tabular}{|lll|}
\hline Page 4: $[352]$ Formatted & KPachonska & $3 / 17 / 20$ 2:15:00 PM \\
\hline Font: $12 \mathrm{pt}$ & & \\
\hline Page 4: $[352]$ Formatted & KPachonska & $3 / 17 / 20$ 2:15:00 PM \\
\hline
\end{tabular}

Font: $12 \mathrm{pt}$

\begin{tabular}{|lll|}
\hline Page 4: $[353]$ Formatted & KPachonska & $3 / 17 / 20$ 2:15:00 PM \\
\hline Font: $12 \mathrm{pt}$ & & \\
\hline Page 4: $[354]$ Deleted & KPachonska & $3 / 17 / 20$ 2:15:00 PM \\
\hline
\end{tabular}

\begin{tabular}{|c|c|c|}
\hline Page 4: [355] Formatted & KPachonska & 3/17/20 2:15:00 PM \\
\hline \multicolumn{3}{|l|}{ Font: $12 \mathrm{pt}$} \\
\hline Page 4: $[356]$ Formatted & KPachonska & 3/17/20 2:15:00 PM \\
\hline \multicolumn{3}{|l|}{ Font: $12 \mathrm{pt}$} \\
\hline Page 4: [357] Formatted & KPachonska & 3/17/20 2:15:00 PM \\
\hline \multicolumn{3}{|l|}{ Font: $12 \mathrm{pt}$} \\
\hline Page 4: [358] Deleted & KPachonska & 3/17/20 2:15:00 PM \\
\hline$\nabla \ldots \ldots$ & & \\
\hline Page 4: [359] Formatted & KPachonska & 3/17/20 2:15:00 PM \\
\hline \multicolumn{3}{|l|}{ Font: $12 \mathrm{pt}$} \\
\hline Page 4: [360] Formatted & KPachonska & 3/17/20 2:15:00 PM \\
\hline \multicolumn{3}{|l|}{ Font: $12 \mathrm{pt}$} \\
\hline Page 4: [361] Formatted & KPachonska & 3/17/20 2:15:00 PM \\
\hline \multicolumn{3}{|l|}{ Font: $12 \mathrm{pt}$} \\
\hline Page 4: [362] Formatted & KPachonska & 3/17/20 2:15:00 PM \\
\hline \multicolumn{3}{|l|}{ Font: $12 \mathrm{pt}$} \\
\hline Page 4: [363] Formatted & KPachonska & 3/17/20 2:15:00 PM \\
\hline \multicolumn{3}{|l|}{ Font: $12 \mathrm{pt}$} \\
\hline Page 4: $[364]$ Formatted & KPachonska & 3/17/20 2:15:00 PM \\
\hline \multicolumn{3}{|l|}{ Font: $12 \mathrm{pt}$} \\
\hline Page 4: $[365]$ Formatted & KPachonska & 3/17/20 2:15:00 PM \\
\hline \multicolumn{3}{|c|}{ Font: Times New Roman } \\
\hline Page 4: [365] Formatted & KPachonska & 3/17/20 2:15:00 PM \\
\hline \multicolumn{3}{|c|}{ Font: Times New Roman } \\
\hline Page 4: [365] Formatted & KPachonska & 3/17/20 2:15:00 PM \\
\hline
\end{tabular}

Font: Times New Roman 


\begin{tabular}{|c|c|c|}
\hline Page 4: [366] Formatted & KPachonska & 3/17/20 2:15:00 PM \\
\hline \multicolumn{3}{|l|}{ Formatted } \\
\hline Page 4: [367] Deleted & KPachonska & 3/17/20 2:15:00 PM \\
\hline Page 1: [368] Formatted & KPachonska & 3/17/20 2:15:00 PM \\
\hline \multicolumn{3}{|c|}{ Normal, Tab stops: 6.26", Right } \\
\hline Page 1: [369] Formatted & KPachonska & 3/17/20 2:15:00 PM \\
\hline \multicolumn{3}{|c|}{ Normal, Tab stops: 6.26", Right } \\
\hline Page 5: [370] Formatted & KPachonska & 3/17/20 2:15:00 PM \\
\hline \multicolumn{3}{|c|}{ Normal, Space After: 8 pt, Line spacing: Multiple 1.15 li, Pattern: Clear (White) } \\
\hline Page 5: [371] Deleted & KPachonska & 3/17/20 2:15:00 PM \\
\hline$\checkmark \ldots$ & \multicolumn{2}{|c|}{$\nabla \ldots$} \\
\hline Page 5: [371] Deleted & KPachonska & 3/17/20 2:15:00 PM \\
\hline \multicolumn{3}{|l|}{ 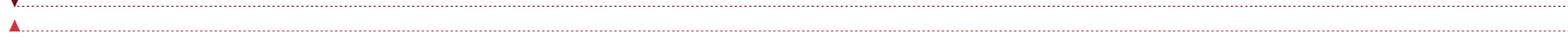 } \\
\hline Page 5: [372] Formatted & KPachonska & 3/17/20 2:15:00 PM \\
\hline \multicolumn{3}{|l|}{ Font: $12 \mathrm{pt}$} \\
\hline Page 5: [373] Formatted & KPachonska & 3/17/20 2:15:00 PM \\
\hline \multicolumn{3}{|l|}{ Font: $12 \mathrm{pt}$} \\
\hline Page 5: [374] Formatted & KPachonska & 3/17/20 2:15:00 PM \\
\hline \multicolumn{3}{|l|}{ Font: $12 \mathrm{pt}$} \\
\hline Page 5: [375] Formatted & KPachonska & 3/17/20 2:15:00 PM \\
\hline \multicolumn{3}{|l|}{ Font: $12 \mathrm{pt}$} \\
\hline Page 5: [376] Deleted & KPachonska & 3/17/20 2:15:00 PM \\
\hline \multicolumn{3}{|l|}{$\checkmark$} \\
\hline Page 5: [377] Formatted & KPachonska & 3/17/20 2:15:00 PM \\
\hline \multicolumn{3}{|l|}{ Font: $12 \mathrm{pt}$} \\
\hline Page 5: [378] Formatted & KPachonska & 3/17/20 2:15:00 PM \\
\hline \multicolumn{3}{|l|}{ Font: $12 \mathrm{pt}$} \\
\hline Page 5: [379] Formatted & KPachonska & 3/17/20 2:15:00 PM \\
\hline \multicolumn{3}{|l|}{ Font: $12 \mathrm{pt}$} \\
\hline Page 5: [380] Formatted & KPachonska & 3/17/20 2:15:00 PM \\
\hline \multicolumn{3}{|l|}{ Font: $12 \mathrm{pt}$} \\
\hline Page 5: [381] Formatted & KPachonska & 3/17/20 2:15:00 PM \\
\hline \multicolumn{3}{|l|}{ Font: $12 \mathrm{pt}$} \\
\hline Page 5: [382] Formatted & KPachonska & 3/17/20 2:15:00 PM \\
\hline \multicolumn{3}{|l|}{ Font: $12 \mathrm{pt}$} \\
\hline Page 5: [383] Formatted & KPachonska & 3/17/20 2:15:00 PM \\
\hline
\end{tabular}




\begin{tabular}{|c|c|c|}
\hline Page 5: [384] Formatted & KPachonska & 3/17/20 2:15:00 PM \\
\hline \multicolumn{3}{|l|}{ Font: $12 \mathrm{pt}$} \\
\hline Page 5: [385] Formatted & KPachonska & 3/17/20 2:15:00 PM \\
\hline \multicolumn{3}{|l|}{ Font: $12 \mathrm{pt}$} \\
\hline Page 5: [386] Formatted & KPachonska & 3/17/20 2:15:00 PM \\
\hline \multicolumn{3}{|l|}{ Font: $12 \mathrm{pt}$} \\
\hline Page 5: [387] Formatted & KPachonska & 3/17/20 2:15:00 PM \\
\hline \multicolumn{3}{|l|}{ Font: $12 \mathrm{pt}$} \\
\hline Page 5: [388] Formatted & KPachonska & 3/17/20 2:15:00 PM \\
\hline \multicolumn{3}{|l|}{ Font: $12 \mathrm{pt}$} \\
\hline Page 5: [389] Formatted & KPachonska & 3/17/20 2:15:00 PM \\
\hline \multicolumn{3}{|l|}{ Font: $12 \mathrm{pt}$} \\
\hline Page 5: [390] Formatted & KPachonska & 3/17/20 2:15:00 PM \\
\hline \multicolumn{3}{|l|}{ Font: $12 \mathrm{pt}$} \\
\hline Page 5: [391] Formatted & KPachonska & 3/17/20 2:15:00 PM \\
\hline \multicolumn{3}{|l|}{ Font: $12 \mathrm{pt}$} \\
\hline Page 5: [392] Formatted & KPachonska & 3/17/20 2:15:00 PM \\
\hline \multicolumn{3}{|l|}{ Font: $12 \mathrm{pt}$} \\
\hline Page 5: [393] Formatted & KPachonska & 3/17/20 2:15:00 PM \\
\hline \multicolumn{3}{|l|}{ Font: $12 \mathrm{pt}$} \\
\hline Page 5: [394] Formatted & KPachonska & 3/17/20 2:15:00 PM \\
\hline \multicolumn{3}{|l|}{ Font: $12 \mathrm{pt}$} \\
\hline Page 5: [395] Formatted & KPachonska & 3/17/20 2:15:00 PM \\
\hline \multicolumn{3}{|l|}{ Font: $12 \mathrm{pt}$} \\
\hline Page 5: [396] Formatted & KPachonska & 3/17/20 2:15:00 PM \\
\hline \multicolumn{3}{|l|}{ Font: $12 \mathrm{pt}$} \\
\hline Page 5: [397] Formatted & KPachonska & 3/17/20 2:15:00 PM \\
\hline \multicolumn{3}{|l|}{ Font: $12 \mathrm{pt}$} \\
\hline Page 5: [398] Formatted & KPachonska & 3/17/20 2:15:00 PM \\
\hline \multicolumn{3}{|l|}{ Font: $12 \mathrm{pt}$} \\
\hline Page 5: [399] Formatted & KPachonska & 3/17/20 2:15:00 PM \\
\hline \multicolumn{3}{|l|}{ Font: $12 \mathrm{pt}$} \\
\hline Page 5: $[400]$ Formatted & KPachonska & 3/17/20 2:15:00 PM \\
\hline \multicolumn{3}{|l|}{ Font: $12 \mathrm{pt}$} \\
\hline Page 5: [401] Formatted & KPachonska & 3/17/20 2:15:00 PM \\
\hline \multicolumn{3}{|l|}{ Font: $12 \mathrm{pt}$} \\
\hline Page 5: $[402]$ Formatted & KPachonska & 3/17/20 2:15:00 PM \\
\hline \multicolumn{3}{|l|}{ Font: $12 \mathrm{pt}$} \\
\hline Page 5: [403] Formatted & KPachonska & 3/17/20 2:15:00 PM \\
\hline
\end{tabular}

Font: $12 \mathrm{pt}$ 


\begin{tabular}{|c|c|c|}
\hline Page 5: [404] Formatted & KPachonska & 3/17/20 2:15:00 PM \\
\hline \multicolumn{3}{|l|}{ Font: $12 \mathrm{pt}$} \\
\hline Page 5: [405] Formatted & KPachonska & 3/17/20 2:15:00 PM \\
\hline \multicolumn{3}{|l|}{ Font: $12 \mathrm{pt}$} \\
\hline Page 5: $[406]$ Formatted & KPachonska & 3/17/20 2:15:00 PM \\
\hline \multicolumn{3}{|l|}{ Font: $12 \mathrm{pt}$} \\
\hline Page 5: [407] Formatted & KPachonska & 3/17/20 2:15:00 PM \\
\hline \multicolumn{3}{|l|}{ Font: $12 \mathrm{pt}$} \\
\hline Page 5: [408] Formatted & KPachonska & 3/17/20 2:15:00 PM \\
\hline \multicolumn{3}{|l|}{ Font: $12 \mathrm{pt}$} \\
\hline Page 5: [409] Formatted & KPachonska & 3/17/20 2:15:00 PM \\
\hline \multicolumn{3}{|l|}{ Font: $12 \mathrm{pt}$} \\
\hline Page 5: [410] Formatted & KPachonska & 3/17/20 2:15:00 PM \\
\hline \multicolumn{3}{|l|}{ Font: $12 \mathrm{pt}$} \\
\hline Page 5: [411] Formatted & KPachonska & 3/17/20 2:15:00 PM \\
\hline \multicolumn{3}{|l|}{ Font: $12 \mathrm{pt}$} \\
\hline Page 5: [412] Formatted & KPachonska & 3/17/20 2:15:00 PM \\
\hline \multicolumn{3}{|l|}{ Font: $12 \mathrm{pt}$} \\
\hline Page 5: [413] Formatted & KPachonska & 3/17/20 2:15:00 PM \\
\hline \multicolumn{3}{|l|}{ Font: $12 \mathrm{pt}$} \\
\hline Page 5: [414] Formatted & KPachonska & 3/17/20 2:15:00 PM \\
\hline \multicolumn{3}{|l|}{ Font: $12 \mathrm{pt}$} \\
\hline Page 5: [415] Formatted & KPachonska & 3/17/20 2:15:00 PM \\
\hline \multicolumn{3}{|l|}{ Font: $12 \mathrm{pt}$} \\
\hline Page 5: [416] Formatted & KPachonska & 3/17/20 2:15:00 PM \\
\hline \multicolumn{3}{|l|}{ Font: $12 \mathrm{pt}$} \\
\hline Page 5: [417] Formatted & KPachonska & 3/17/20 2:15:00 PM \\
\hline \multicolumn{3}{|l|}{ Font: $12 \mathrm{pt}$} \\
\hline Page 5: [418] Formatted & KPachonska & 3/17/20 2:15:00 PM \\
\hline \multicolumn{3}{|l|}{ Font: $12 \mathrm{pt}$} \\
\hline Page 5: [419] Formatted & KPachonska & 3/17/20 2:15:00 PM \\
\hline \multicolumn{3}{|l|}{ Font: $12 \mathrm{pt}$} \\
\hline Page 5: [420] Formatted & KPachonska & 3/17/20 2:15:00 PM \\
\hline \multicolumn{3}{|l|}{ Font: $12 \mathrm{pt}$} \\
\hline Page 5: [421] Deleted & KPachonska & 3/17/20 2:15:00 PM \\
\hline & & \\
\hline Page 5: [422] Formatted & KPachonska & 3/17/20 2:15:00 PM \\
\hline \multicolumn{3}{|l|}{ Font: $12 \mathrm{pt}$} \\
\hline Page 5: [423] Formatted & KPachonska & 3/17/20 2:15:00 PM \\
\hline
\end{tabular}


Font: $12 \mathrm{pt}$

Page 5: [424] Formatted

KPachonska

3/17/20 2:15:00 PM

Font: $12 \mathrm{pt}$

Page 5: [425] Formatted

KPachonska

3/17/20 2:15:00 PM

Font: $12 \mathrm{pt}$

Page 5: [426] Formatted

KPachonska

3/17/20 2:15:00 PM

Font: $12 \mathrm{pt}$

Page 5: [426] Formatted

KPachonska

3/17/20 2:15:00 PM

Font: $12 \mathrm{pt}$

Page 5: [427] Formatted

KPachonska

3/17/20 2:15:00 PM

Font: $12 \mathrm{pt}$

Page 5: [428] Formatted

KPachonska

3/17/20 2:15:00 PM

Font: $12 \mathrm{pt}$

Page 5: [429] Formatted

KPachonska

3/17/20 2:15:00 PM

Font: $12 \mathrm{pt}$

Page 5: [430] Formatted

KPachonska

3/17/20 2:15:00 PM

Font: $12 \mathrm{pt}$

Page 5: [431] Formatted

KPachonska

3/17/20 2:15:00 PM

Font: $12 \mathrm{pt}$

Page 5: [432] Formatted

KPachonska

3/17/20 2:15:00 PM

Font: $12 \mathrm{pt}$

Page 5: [433] Formatted

KPachonska

3/17/20 2:15:00 PM

Font: $12 \mathrm{pt}$

Page 5: [434] Formatted

KPachonska

3/17/20 2:15:00 PM

Font: $12 \mathrm{pt}$

Page 5: [435] Formatted

KPachonska

3/17/20 2:15:00 PM

Font: $12 \mathrm{pt}$

Page 5: [436] Formatted

KPachonska

3/17/20 2:15:00 PM

Font: $12 \mathrm{pt}$

Page 5: [437] Formatted

KPachonska

3/17/20 2:15:00 PM

Font: $12 \mathrm{pt}$

Page 5: [438] Formatted

KPachonska

3/17/20 2:15:00 PM

Font: $12 \mathrm{pt}$

\begin{tabular}{|c|c|c|}
\hline Page 5: [439] Formatted & KPachonska & 3/17/20 2:15:00 PM \\
\hline \multicolumn{3}{|l|}{ Font: $12 \mathrm{pt}$} \\
\hline Page 5: [440] Formatted & KPachonska & 3/17/20 2:15:00 PM \\
\hline \multicolumn{3}{|l|}{ Font: $12 \mathrm{pt}$} \\
\hline Page 5: [441] Formatted & KPachonska & 3/17/20 2:15:00 PM \\
\hline \multicolumn{3}{|l|}{ Font: $12 \mathrm{pt}$} \\
\hline Page 5: [442] Formatted & KPachonska & 3/17/20 2:15:00 PM \\
\hline
\end{tabular}


Font: $12 \mathrm{pt}$

Page 5: [443] Formatted

KPachonska

3/17/20 2:15:00 PM

Font: $12 \mathrm{pt}$

Page 5: [444] Formatted

KPachonska

3/17/20 2:15:00 PM

Font: $12 \mathrm{pt}$

Page 5: [445] Formatted

KPachonska

3/17/20 2:15:00 PM

Font: $12 \mathrm{pt}$

Page 5: [446] Formatted

KPachonska

3/17/20 2:15:00 PM

Font: $12 \mathrm{pt}$

Page 5: [447] Formatted

KPachonska

3/17/20 2:15:00 PM

Font: $12 \mathrm{pt}$

Page 5: [447] Formatted

KPachonska

3/17/20 2:15:00 PM

Font: $12 \mathrm{pt}$

Page 5: [448] Formatted

KPachonska

3/17/20 2:15:00 PM

Font: $12 \mathrm{pt}$

Page 5: [449] Formatted

KPachonska

3/17/20 2:15:00 PM

Font: $12 \mathrm{pt}$

Page 5: [450] Formatted

KPachonska

3/17/20 2:15:00 PM

Font: $12 \mathrm{pt}$

Page 5: [450] Formatted

KPachonska

3/17/20 2:15:00 PM

Font: $12 \mathrm{pt}$

Page 5: [451] Formatted

KPachonska

3/17/20 2:15:00 PM

Font: $12 \mathrm{pt}$

Page 5: [452] Formatted

KPachonska

3/17/20 2:15:00 PM

Font: $12 \mathrm{pt}$

Page 5: [453] Formatted

KPachonska

3/17/20 2:15:00 PM

Font: $12 \mathrm{pt}$

Page 5: [454] Formatted

KPachonska

3/17/20 2:15:00 PM

Font: $12 \mathrm{pt}$

Page 5: [455] Formatted

KPachonska

3/17/20 2:15:00 PM

Font: $12 \mathrm{pt}$

Page 5: [456] Formatted

KPachonska

3/17/20 2:15:00 PM

Font: $12 \mathrm{pt}$

\begin{tabular}{|llr|}
\hline Page 5: $[457]$ Formatted & KPachonska & $3 / 17 / 20$ 2:15:00 PM \\
\hline Font: $12 \mathrm{pt}$ & & \\
\hline Page 5: $[458]$ Formatted & KPachonska & $3 / 17 / 20$ 2:15:00 PM \\
\hline Font: $12 \mathrm{pt}$ & & $3 / 17 / 202: 15: 00$ PM \\
\hline Page 5: $[459]$ Deleted & KPachonska & \\
\hline
\end{tabular}




\begin{tabular}{|c|c|c|}
\hline Page 5: [460] Formatted & KPachonska & 3/17/20 2:15:00 PM \\
\hline \multicolumn{3}{|l|}{ Font: $12 \mathrm{pt}$} \\
\hline Page 5: [461] Formatted & KPachonska & 3/17/20 2:15:00 PM \\
\hline \multicolumn{3}{|l|}{ Font: $12 \mathrm{pt}$} \\
\hline Page 5: [462] Formatted & KPachonska & 3/17/20 2:15:00 PM \\
\hline \multicolumn{3}{|l|}{ Font: $12 \mathrm{pt}$} \\
\hline Page 5: [463] Formatted & KPachonska & 3/17/20 2:15:00 PM \\
\hline \multicolumn{3}{|l|}{ Font: $12 \mathrm{pt}$} \\
\hline Page 5: [464] Formatted & KPachonska & 3/17/20 2:15:00 PM \\
\hline \multicolumn{3}{|l|}{ Font: $12 \mathrm{pt}$} \\
\hline Page 5: [465] Formatted & KPachonska & 3/17/20 2:15:00 PM \\
\hline \multicolumn{3}{|l|}{ Font: $12 \mathrm{pt}$} \\
\hline Page 5: [466] Formatted & KPachonska & 3/17/20 2:15:00 PM \\
\hline \multicolumn{3}{|l|}{ Font: $12 \mathrm{pt}$} \\
\hline Page 5: [467] Formatted & KPachonska & 3/17/20 2:15:00 PM \\
\hline \multicolumn{3}{|l|}{ Font: $12 \mathrm{pt}$} \\
\hline Page 5: [468] Formatted & KPachonska & 3/17/20 2:15:00 PM \\
\hline \multicolumn{3}{|l|}{ Font: $12 \mathrm{pt}$} \\
\hline Page 5: [469] Formatted & KPachonska & 3/17/20 2:15:00 PM \\
\hline \multicolumn{3}{|l|}{ Font: $12 \mathrm{pt}$} \\
\hline Page 5: [470] Formatted & KPachonska & 3/17/20 2:15:00 PM \\
\hline \multicolumn{3}{|l|}{ Font: $12 \mathrm{pt}$} \\
\hline Page 5: [471] Formatted & KPachonska & 3/17/20 2:15:00 PM \\
\hline \multicolumn{3}{|l|}{ Font: $12 \mathrm{pt}$} \\
\hline Page 5: [472] Formatted & KPachonska & 3/17/20 2:15:00 PM \\
\hline \multicolumn{3}{|l|}{ Font: $12 \mathrm{pt}$} \\
\hline Page 5: [473] Formatted & KPachonska & 3/17/20 2:15:00 PM \\
\hline \multicolumn{3}{|l|}{ Font: $12 \mathrm{pt}$} \\
\hline Page 5: [474] Formatted & KPachonska & 3/17/20 2:15:00 PM \\
\hline \multicolumn{3}{|l|}{ Font: $12 \mathrm{pt}$} \\
\hline Page 5: [475] Formatted & KPachonska & 3/17/20 2:15:00 PM \\
\hline \multicolumn{3}{|l|}{ Font: $12 \mathrm{pt}$} \\
\hline Page 5: [476] Formatted & KPachonska & 3/17/20 2:15:00 PM \\
\hline \multicolumn{3}{|l|}{ Font: $12 \mathrm{pt}$} \\
\hline Page 5: [477] Formatted & KPachonska & 3/17/20 2:15:00 PM \\
\hline \multicolumn{3}{|l|}{ Font: $12 \mathrm{pt}$} \\
\hline Page 5: $[478]$ Formatted & KPachonska & 3/17/20 2:15:00 PM \\
\hline \multicolumn{3}{|l|}{ Font: $12 \mathrm{pt}$} \\
\hline Page 5: [479] Formatted & KPachonska & 3/17/20 2:15:00 PM \\
\hline
\end{tabular}

Font: $12 \mathrm{pt}$ 


\begin{tabular}{|c|c|c|}
\hline Page 5: [480] Formatted & KPachonska & 3/17/20 2:15:00 PM \\
\hline \multicolumn{3}{|l|}{ Font: $12 \mathrm{pt}$} \\
\hline Page 5: [481] Formatted & KPachonska & 3/17/20 2:15:00 PM \\
\hline \multicolumn{3}{|l|}{ Font: $12 \mathrm{pt}$} \\
\hline Page 5: [482] Formatted & KPachonska & 3/17/20 2:15:00 PM \\
\hline \multicolumn{3}{|l|}{ Font: $12 \mathrm{pt}$} \\
\hline Page 5: [483] Formatted & KPachonska & 3/17/20 2:15:00 PM \\
\hline \multicolumn{3}{|l|}{ Font: $12 \mathrm{pt}$} \\
\hline Page 5: [484] Formatted & KPachonska & 3/17/20 2:15:00 PM \\
\hline \multicolumn{3}{|l|}{ Font: $12 \mathrm{pt}$} \\
\hline Page 5: [485] Formatted & KPachonska & 3/17/20 2:15:00 PM \\
\hline \multicolumn{3}{|l|}{ Font: $12 \mathrm{pt}$} \\
\hline Page 5: [486] Formatted & KPachonska & 3/17/20 2:15:00 PM \\
\hline \multicolumn{3}{|l|}{ Font: $12 \mathrm{pt}$} \\
\hline Page 5: [487] Formatted & KPachonska & 3/17/20 2:15:00 PM \\
\hline \multicolumn{3}{|c|}{ Normal, Space After: 8 pt, Line spacing: Multiple 1.15 li, Pattern: Clear (White) } \\
\hline Page 5: [488] Formatted & KPachonska & 3/17/20 2:15:00 PM \\
\hline \multicolumn{3}{|l|}{ Font: $12 \mathrm{pt}$} \\
\hline Page 5: [489] Formatted & KPachonska & 3/17/20 2:15:00 PM \\
\hline \multicolumn{3}{|l|}{ Font: $12 \mathrm{pt}$} \\
\hline Page 5: [490] Formatted & KPachonska & 3/17/20 2:15:00 PM \\
\hline \multicolumn{3}{|l|}{ Font: $12 \mathrm{pt}$} \\
\hline Page 5: [491] Formatted & KPachonska & 3/17/20 2:15:00 PM \\
\hline \multicolumn{3}{|l|}{ Font: $12 \mathrm{pt}$} \\
\hline Page 5: [492] Formatted & KPachonska & 3/17/20 2:15:00 PM \\
\hline \multicolumn{3}{|l|}{ Font: $12 \mathrm{pt}$} \\
\hline Page 5: [493] Formatted & KPachonska & 3/17/20 2:15:00 PM \\
\hline \multicolumn{3}{|l|}{ Font: $12 \mathrm{pt}$} \\
\hline Page 5: [494] Formatted & KPachonska & 3/17/20 2:15:00 PM \\
\hline \multicolumn{3}{|l|}{ Font: $12 \mathrm{pt}$} \\
\hline Page 5: [495] Formatted & KPachonska & 3/17/20 2:15:00 PM \\
\hline \multicolumn{3}{|l|}{ Font: $12 \mathrm{pt}$} \\
\hline Page 5: [496] Formatted & KPachonska & 3/17/20 2:15:00 PM \\
\hline \multicolumn{3}{|l|}{ Font: $12 \mathrm{pt}$} \\
\hline Page 5: [497] Formatted & KPachonska & 3/17/20 2:15:00 PM \\
\hline \multicolumn{3}{|l|}{ Font: $12 \mathrm{pt}$} \\
\hline Page 5: [498] Formatted & KPachonska & 3/17/20 2:15:00 PM \\
\hline \multicolumn{3}{|l|}{ Font: $12 \mathrm{pt}$} \\
\hline Page 5: [499] Formatted & KPachonska & 3/17/20 2:15:00 PM \\
\hline
\end{tabular}




\begin{tabular}{|c|c|c|}
\hline Page 5: [500] Formatted & KPachonska & 3/17/20 2:15:00 PM \\
\hline \multicolumn{3}{|l|}{ Font: $12 \mathrm{pt}$} \\
\hline Page 5: [501] Formatted & KPachonska & 3/17/20 2:15:00 PM \\
\hline \multicolumn{3}{|l|}{ Font: $12 \mathrm{pt}$} \\
\hline Page 5: [501] Formatted & KPachonska & 3/17/20 2:15:00 PM \\
\hline \multicolumn{3}{|l|}{ Font: $12 \mathrm{pt}$} \\
\hline Page 5: [502] Formatted & KPachonska & 3/17/20 2:15:00 PM \\
\hline \multicolumn{3}{|l|}{ Font: $12 \mathrm{pt}$} \\
\hline Page 5: [503] Formatted & KPachonska & 3/17/20 2:15:00 PM \\
\hline \multicolumn{3}{|l|}{ Font: $12 \mathrm{pt}$} \\
\hline Page 5: [504] Formatted & KPachonska & 3/17/20 2:15:00 PM \\
\hline \multicolumn{3}{|l|}{ Font: $12 \mathrm{pt}$} \\
\hline Page 5: [504] Formatted & KPachonska & 3/17/20 2:15:00 PM \\
\hline \multicolumn{3}{|l|}{ Font: $12 \mathrm{pt}$} \\
\hline Page 1: [505] Formatted & KPachonska & 3/17/20 2:15:00 PM \\
\hline \multicolumn{3}{|c|}{ Normal, Tab stops: $6.26 "$, Right } \\
\hline Page 1: [506] Formatted & KPachonska & 3/17/20 2:15:00 PM \\
\hline \multicolumn{3}{|c|}{ Normal, Tab stops: $6.26 "$, Right } \\
\hline Page 6: [507] Formatted & KPachonska & 3/17/20 2:15:00 PM \\
\hline \multicolumn{3}{|l|}{ Font: $12 \mathrm{pt}$} \\
\hline Page 6: [508] Formatted & KPachonska & 3/17/20 2:15:00 PM \\
\hline \multicolumn{3}{|l|}{ Font: $12 \mathrm{pt}$} \\
\hline Page 6: [509] Formatted & KPachonska & 3/17/20 2:15:00 PM \\
\hline \multicolumn{3}{|l|}{ Font: $12 \mathrm{pt}$} \\
\hline Page 6: $[510]$ Formatted & KPachonska & 3/17/20 2:15:00 PM \\
\hline \multicolumn{3}{|l|}{ Font: $12 \mathrm{pt}$} \\
\hline Page 6: [511] Formatted & KPachonska & 3/17/20 2:15:00 PM \\
\hline \multicolumn{3}{|l|}{ Font: $12 \mathrm{pt}$} \\
\hline Page 6: [512] Formatted & KPachonska & 3/17/20 2:15:00 PM \\
\hline \multicolumn{3}{|l|}{ Font: $12 \mathrm{pt}$} \\
\hline Page 6: [513] Formatted & KPachonska & 3/17/20 2:15:00 PM \\
\hline \multicolumn{3}{|l|}{ Font: $12 \mathrm{pt}$} \\
\hline Page 6: [514] Formatted & KPachonska & 3/17/20 2:15:00 PM \\
\hline \multicolumn{3}{|l|}{ Font: $12 \mathrm{pt}$} \\
\hline Page 6: [515] Formatted & KPachonska & 3/17/20 2:15:00 PM \\
\hline \multicolumn{3}{|l|}{ Font: $12 \mathrm{pt}$} \\
\hline Page 6: [516] Formatted & KPachonska & 3/17/20 2:15:00 PM \\
\hline \multicolumn{3}{|l|}{ Font: $12 \mathrm{pt}$} \\
\hline Page 6: [517] Formatted & KPachonska & 3/17/20 2:15:00 PM \\
\hline
\end{tabular}

Font: $12 \mathrm{pt}$ 


\begin{tabular}{|c|c|c|}
\hline Page 6: [518] Formatted & KPachonska & 3/17/20 2:15:00 PM \\
\hline \multicolumn{3}{|l|}{ Font: $12 \mathrm{pt}$} \\
\hline Page 6: [519] Formatted & KPachonska & 3/17/20 2:15:00 PM \\
\hline \multicolumn{3}{|l|}{ Font: $12 \mathrm{pt}$} \\
\hline Page 6: [520] Formatted & KPachonska & 3/17/20 2:15:00 PM \\
\hline \multicolumn{3}{|l|}{ Font: $12 \mathrm{pt}$} \\
\hline Page 6: [521] Formatted & KPachonska & 3/17/20 2:15:00 РM \\
\hline \multicolumn{3}{|l|}{ Font: $12 \mathrm{pt}$} \\
\hline Page 6: [522] Formatted & KPachonska & 3/17/20 2:15:00 PM \\
\hline \multicolumn{3}{|l|}{ Font: $12 \mathrm{pt}$} \\
\hline Page 6: [523] Formatted & KPachonska & 3/17/20 2:15:00 PM \\
\hline \multicolumn{3}{|l|}{ Font: $12 \mathrm{pt}$} \\
\hline Page 6: [524] Formatted & KPachonska & 3/17/20 2:15:00 PM \\
\hline \multicolumn{3}{|l|}{ Font: $12 \mathrm{pt}$} \\
\hline Page 6: [525] Formatted & KPachonska & 3/17/20 2:15:00 PM \\
\hline \multicolumn{3}{|l|}{ Font: $12 \mathrm{pt}$} \\
\hline Page 6: [526] Formatted & KPachonska & 3/17/20 2:15:00 PM \\
\hline \multicolumn{3}{|l|}{ Font: $12 \mathrm{pt}$} \\
\hline Page 6: [527] Formatted & KPachonska & 3/17/20 2:15:00 PM \\
\hline \multicolumn{3}{|l|}{ Font: $12 \mathrm{pt}$} \\
\hline Page 6: [528] Formatted & KPachonska & 3/17/20 2:15:00 PM \\
\hline \multicolumn{3}{|l|}{ Font: $12 \mathrm{pt}$} \\
\hline Page 6: [529] Formatted & KPachonska & 3/17/20 2:15:00 PM \\
\hline \multicolumn{3}{|l|}{ Font: $12 \mathrm{pt}$} \\
\hline Page 6: [530] Deleted & KPachonska & 3/17/20 2:15:00 PM \\
\hline \multicolumn{3}{|l|}{$\nabla \ldots$} \\
\hline Page 6: [530] Deleted & KPachonska & 3/17/20 2:15:00 PM \\
\hline \multicolumn{3}{|l|}{$\checkmark$} \\
\hline Page 6: [530] Deleted & KPachonska & 3/17/20 2:15:00 PM \\
\hline \multicolumn{3}{|l|}{$\nabla$} \\
\hline Page 6: [530] Deleted & KPachonska & 3/17/20 2:15:00 PM \\
\hline \multirow{2}{*}{\multicolumn{3}{|c|}{ 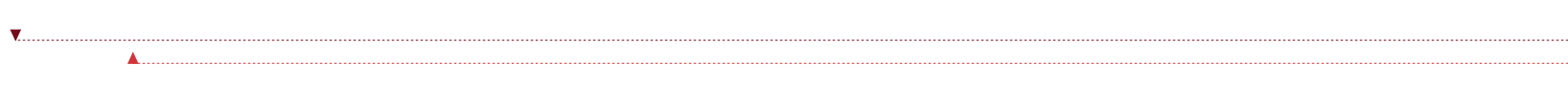 }} \\
\hline & & \\
\hline Page 6: [530] Deleted & KPachonska & 3/17/20 2:15:00 PM \\
\hline$\nabla$ & & \\
\hline Page 6: [530] Deleted & KPachonska & 3/17/20 2:15:00 PM \\
\hline
\end{tabular}




\section{Page 6: [530] Deleted}

Page 6: [530] Deleted

KPachonska

3/17/20 2:15:00 PM

Page 6: [530] Deleted

KPachonska

3/17/20 2:15:00 PM

$\checkmark$

Page 6: [530] Deleted

KPachonska

3/17/20 2:15:00 PM

Page 6: [530] Deleted

KPachonska

3/17/20 2:15:00 PM

Page 6: [530] Deleted

KPachonska

3/17/20 2:15:00 PM

Page 6: [530] Deleted

KPachonska

3/17/20 2:15:00 PM

Page 6: [530] Deleted

KPachonska

3/17/20 2:15:00 PM

Page 6: [530] Deleted

KPachonska

3/17/20 2:15:00 PM

$\checkmark$

Page 6: [530] Deleted

KPachonska

3/17/20 2:15:00 PM

$\checkmark$

Page 6: [530] Deleted

KPachonska

3/17/20 2:15:00 PM

$\checkmark$

Page 6: [530] Deleted

KPachonska

3/17/20 2:15:00 PM

Page 6: [530] Deleted

KPachonska

3/17/20 2:15:00 PM

$\checkmark$

Page 6: [530] Deleted

KPachonska

3/17/20 2:15:00 PM

$\checkmark$

Page 6: [530] Deleted

KPachonska

3/17/20 2:15:00 PM 


\begin{tabular}{|c|c|c|}
\hline Page 6: [530] Deleted & KPachonska & 3/17/20 2:15:00 PM \\
\hline v. & & \\
\hline Page 6: [530] Deleted & KPachonska & 3/17/20 2:15:00 PM \\
\hline$\nabla$. & & \\
\hline Page 6: [530] Deleted & KPachonska & 3/17/20 2:15:00 PM \\
\hline$\nabla$ & & \\
\hline Page 6: [530] Deleted & KPachonska & 3/17/20 2:15:00 PM \\
\hline$\checkmark$ & & \\
\hline Page 6: [530] Deleted & KPachonska & 3/17/20 2:15:00 PM \\
\hline$\nabla$ & & \\
\hline $\boldsymbol{\Lambda}$ & & \\
\hline Page 6: [530] Deleted & KPachonska & 3/17/20 2:15:00 PM \\
\hline$\checkmark$ & & \\
\hline $\boldsymbol{\Lambda}$ & 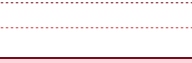 & $1+1-1-1-1-1$ \\
\hline Page 6: [530] Deleted & KPachonska & 3/17/20 2:15:00 PM \\
\hline$\checkmark \ldots$ & & \\
\hline $\boldsymbol{4}$ & & \\
\hline Page 6: [530] Deleted & KPachonska & 3/17/20 2:15:00 PM \\
\hline$\checkmark$ & & \\
\hline $\boldsymbol{\Lambda}$ & 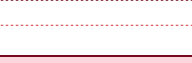 & 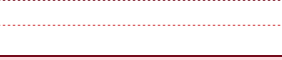 \\
\hline Page 6: [530] Deleted & KPachonska & 3/17/20 2:15:00 PM \\
\hline$\nabla$ & & \\
\hline Page 6: [530] Deleted & KPachonska & 3/17/20 2:15:00 PM \\
\hline & & \\
\hline - & 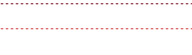 & $v_{\cdots} \cdots$ \\
\hline Page 6: [530] Deleted & KPachonska & 3/17/20 2:15:00 PM \\
\hline$\nabla$ & & \\
\hline $\boldsymbol{\Lambda}$ & 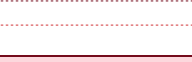 & 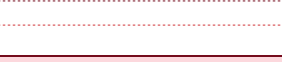 \\
\hline Page 6: [531] Formatted & KPachonska & 3/17/20 2:15:00 PM \\
\hline Font: Not Bold & & \\
\hline Page 6: [531] Formatted & KPachonska & 3/17/20 2:15:00 PM \\
\hline Font: Not Bold & & \\
\hline Page 6: [532] Deleted & KPachonska & 3/17/20 2:15:00 PM \\
\hline$\checkmark$ & & \\
\hline $\boldsymbol{\Lambda}$ & $(\ldots \ldots \ldots \ldots \ldots \ldots \ldots \ldots \ldots \ldots$ & 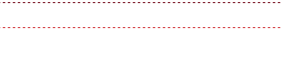 \\
\hline Page 6: [533] Formatted & KPachonska & 3/17/20 2:15:00 PM \\
\hline Font: $12 \mathrm{pt}$ & & \\
\hline Page 6: [534] Formatted & KPachonska & 3/17/20 2:15:00 PM \\
\hline
\end{tabular}

Normal, Space After: 8 pt, Line spacing: Multiple 1.15 li, Pattern: Clear (White) 


\begin{tabular}{|c|c|c|}
\hline Page 6: [535] Formatted & KPachonska & 3/17/20 2:15:00 PM \\
\hline \multicolumn{3}{|l|}{ Font: $12 \mathrm{pt}$} \\
\hline Page 6: [536] Formatted & KPachonska & 3/17/20 2:15:00 PM \\
\hline \multicolumn{3}{|l|}{ Font: $12 \mathrm{pt}$} \\
\hline Page 6: [537] Formatted & KPachonska & 3/17/20 2:15:00 PM \\
\hline \multicolumn{3}{|l|}{ Font: $12 \mathrm{pt}$} \\
\hline Page 6: [538] Formatted & KPachonska & 3/17/20 2:15:00 PM \\
\hline \multicolumn{3}{|l|}{ Font: $12 \mathrm{pt}$} \\
\hline Page 6: [539] Formatted & KPachonska & 3/17/20 2:15:00 PM \\
\hline \multicolumn{3}{|l|}{ Font: $12 \mathrm{pt}$} \\
\hline Page 6: [540] Formatted & KPachonska & 3/17/20 2:15:00 PM \\
\hline \multicolumn{3}{|l|}{ Font: $12 \mathrm{pt}$} \\
\hline Page 6: [541] Formatted & KPachonska & 3/17/20 2:15:00 PM \\
\hline \multicolumn{3}{|l|}{ Font: $12 \mathrm{pt}$} \\
\hline Page 6: [542] Formatted & KPachonska & 3/17/20 2:15:00 PM \\
\hline \multicolumn{3}{|l|}{ Font: $12 \mathrm{pt}$} \\
\hline Page 6: [543] Formatted & KPachonska & 3/17/20 2:15:00 PM \\
\hline \multicolumn{3}{|l|}{ Font: $12 \mathrm{pt}$} \\
\hline Page 6: [544] Formatted & KPachonska & 3/17/20 2:15:00 PM \\
\hline \multicolumn{3}{|l|}{ Font: $12 \mathrm{pt}$} \\
\hline Page 6: [545] Formatted & KPachonska & 3/17/20 2:15:00 PM \\
\hline \multicolumn{3}{|l|}{ Font: $12 \mathrm{pt}$} \\
\hline Page 6: [546] Formatted & KPachonska & 3/17/20 2:15:00 PM \\
\hline \multicolumn{3}{|l|}{ Font: $12 \mathrm{pt}$} \\
\hline Page 6: [547] Formatted & KPachonska & 3/17/20 2:15:00 PM \\
\hline \multicolumn{3}{|l|}{ Font: $12 \mathrm{pt}$} \\
\hline Page 6: [548] Formatted & KPachonska & 3/17/20 2:15:00 PM \\
\hline \multicolumn{3}{|l|}{ Font: $12 \mathrm{pt}$} \\
\hline Page 6: [549] Formatted & KPachonska & 3/17/20 2:15:00 PM \\
\hline \multicolumn{3}{|l|}{ Font: $12 \mathrm{pt}$} \\
\hline Page 6: [550] Formatted & KPachonska & 3/17/20 2:15:00 PM \\
\hline \multicolumn{3}{|l|}{ Font: $12 \mathrm{pt}$} \\
\hline Page 6: [551] Formatted & KPachonska & 3/17/20 2:15:00 PM \\
\hline \multicolumn{3}{|l|}{ Font: $12 \mathrm{pt}$} \\
\hline Page 6: [552] Formatted & KPachonska & 3/17/20 2:15:00 PM \\
\hline \multicolumn{3}{|l|}{ Font: $12 \mathrm{pt}$} \\
\hline Page 6: [553] Formatted & KPachonska & 3/17/20 2:15:00 PM \\
\hline \multicolumn{3}{|l|}{ Font: $12 \mathrm{pt}$} \\
\hline Page 6: [554] Formatted & KPachonska & 3/17/20 2:15:00 PM \\
\hline
\end{tabular}

Font: $12 \mathrm{pt}$ 


\begin{tabular}{|c|c|c|}
\hline Page 6: [555] Formatted & KPachonska & 3/17/20 2:15:00 PM \\
\hline \multicolumn{3}{|l|}{ Font: $12 \mathrm{pt}$} \\
\hline Page 6: [556] Formatted & KPachonska & 3/17/20 2:15:00 PM \\
\hline \multicolumn{3}{|l|}{ Font: $12 \mathrm{pt}$} \\
\hline Page 6: [557] Formatted & KPachonska & 3/17/20 2:15:00 PM \\
\hline \multicolumn{3}{|l|}{ Font: $12 \mathrm{pt}$} \\
\hline Page 6: [558] Formatted & KPachonska & 3/17/20 2:15:00 PM \\
\hline \multicolumn{3}{|l|}{ Font: $12 \mathrm{pt}$} \\
\hline Page 6: [559] Formatted & KPachonska & 3/17/20 2:15:00 PM \\
\hline \multicolumn{3}{|l|}{ Font: $12 \mathrm{pt}$} \\
\hline Page 6: [560] Formatted & KPachonska & 3/17/20 2:15:00 PM \\
\hline \multicolumn{3}{|l|}{ Font: $12 \mathrm{pt}$} \\
\hline Page 6: [561] Formatted & KPachonska & 3/17/20 2:15:00 PM \\
\hline \multicolumn{3}{|l|}{ Font: $12 \mathrm{pt}$} \\
\hline Page 6: [562] Formatted & KPachonska & 3/17/20 2:15:00 PM \\
\hline \multicolumn{3}{|l|}{ Font: $12 \mathrm{pt}$} \\
\hline Page 6: [563] Formatted & KPachonska & 3/17/20 2:15:00 PM \\
\hline \multicolumn{3}{|l|}{ Font: $12 \mathrm{pt}$} \\
\hline Page 6: [564] Formatted & KPachonska & 3/17/20 2:15:00 PM \\
\hline \multicolumn{3}{|l|}{ Font: $12 \mathrm{pt}$} \\
\hline Page 6: [565] Formatted & KPachonska & 3/17/20 2:15:00 PM \\
\hline \multicolumn{3}{|l|}{ Font: $12 \mathrm{pt}$} \\
\hline Page 6: [566] Formatted & KPachonska & 3/17/20 2:15:00 PM \\
\hline \multicolumn{3}{|l|}{ Font: $12 \mathrm{pt}$} \\
\hline Page 6: [567] Formatted & KPachonska & 3/17/20 2:15:00 PM \\
\hline \multicolumn{3}{|l|}{ Font: $12 \mathrm{pt}$} \\
\hline Page 6: [568] Formatted & KPachonska & 3/17/20 2:15:00 PM \\
\hline \multicolumn{3}{|l|}{ Font: $12 \mathrm{pt}$} \\
\hline Page 6: [569] Formatted & KPachonska & 3/17/20 2:15:00 PM \\
\hline \multicolumn{3}{|l|}{ Font: $12 \mathrm{pt}$} \\
\hline Page 6: [570] Formatted & KPachonska & 3/17/20 2:15:00 PM \\
\hline \multicolumn{3}{|l|}{ Font: $12 \mathrm{pt}$} \\
\hline Page 6: [571] Formatted & KPachonska & 3/17/20 2:15:00 PM \\
\hline \multicolumn{3}{|l|}{ Font: $12 \mathrm{pt}$} \\
\hline Page 6: [572] Formatted & KPachonska & 3/17/20 2:15:00 PM \\
\hline \multicolumn{3}{|l|}{ Font: $12 \mathrm{pt}$} \\
\hline Page 6: [573] Formatted & KPachonska & 3/17/20 2:15:00 PM \\
\hline \multicolumn{3}{|l|}{ Font: $12 \mathrm{pt}$} \\
\hline Page 6: [574] Formatted & KPachonska & 3/17/20 2:15:00 PM \\
\hline
\end{tabular}




\begin{tabular}{|c|c|c|}
\hline Page 6: [575] Formatted & KPachonska & 3/17/20 2:15:00 PM \\
\hline \multicolumn{3}{|l|}{ Font: $12 \mathrm{pt}$} \\
\hline Page 6: [576] Formatted & KPachonska & 3/17/20 2:15:00 PM \\
\hline \multicolumn{3}{|l|}{ Font: $12 \mathrm{pt}$} \\
\hline Page 6: [577] Formatted & KPachonska & 3/17/20 2:15:00 PM \\
\hline \multicolumn{3}{|l|}{ Font: $12 \mathrm{pt}$} \\
\hline Page 6: [578] Formatted & KPachonska & 3/17/20 2:15:00 PM \\
\hline \multicolumn{3}{|l|}{ Font: $12 \mathrm{pt}$} \\
\hline Page 6: [579] Formatted & KPachonska & 3/17/20 2:15:00 PM \\
\hline \multicolumn{3}{|l|}{ Font: $12 \mathrm{pt}$} \\
\hline Page 6: [580] Formatted & KPachonska & 3/17/20 2:15:00 PM \\
\hline \multicolumn{3}{|l|}{ Font: $12 \mathrm{pt}$} \\
\hline Page 6: [581] Formatted & KPachonska & 3/17/20 2:15:00 PM \\
\hline \multicolumn{3}{|l|}{ Font: $12 \mathrm{pt}$} \\
\hline Page 6: [582] Formatted & KPachonska & 3/17/20 2:15:00 PM \\
\hline \multicolumn{3}{|l|}{ Font: $12 \mathrm{pt}$} \\
\hline Page 6: [583] Formatted & KPachonska & 3/17/20 2:15:00 PM \\
\hline \multicolumn{3}{|l|}{ Font: $12 \mathrm{pt}$} \\
\hline Page 6: [584] Formatted & KPachonska & 3/17/20 2:15:00 PM \\
\hline \multicolumn{3}{|l|}{ Font: $12 \mathrm{pt}$} \\
\hline Page 6: [585] Formatted & KPachonska & 3/17/20 2:15:00 PM \\
\hline \multicolumn{3}{|l|}{ Font: $12 \mathrm{pt}$} \\
\hline Page 6: [586] Formatted & KPachonska & 3/17/20 2:15:00 PM \\
\hline \multicolumn{3}{|l|}{ Font: $12 \mathrm{pt}$} \\
\hline Page 6: [587] Formatted & KPachonska & 3/17/20 2:15:00 PM \\
\hline \multicolumn{3}{|l|}{ Font: $12 \mathrm{pt}$} \\
\hline Page 6: [588] Formatted & KPachonska & 3/17/20 2:15:00 PM \\
\hline \multicolumn{3}{|l|}{ Font: $12 \mathrm{pt}$} \\
\hline Page 6: [589] Formatted & KPachonska & 3/17/20 2:15:00 PM \\
\hline \multicolumn{3}{|l|}{ Font: $12 \mathrm{pt}$} \\
\hline Page 6: [590] Formatted & KPachonska & 3/17/20 2:15:00 PM \\
\hline \multicolumn{3}{|l|}{ Font: $12 \mathrm{pt}$} \\
\hline Page 6: [591] Formatted & KPachonska & 3/17/20 2:15:00 PM \\
\hline \multicolumn{3}{|l|}{ Font: $12 \mathrm{pt}$} \\
\hline Page 6: [592] Formatted & KPachonska & 3/17/20 2:15:00 PM \\
\hline \multicolumn{3}{|l|}{ Font: $12 \mathrm{pt}$} \\
\hline Page 6: [593] Formatted & KPachonska & 3/17/20 2:15:00 PM \\
\hline \multicolumn{3}{|l|}{ Font: $12 \mathrm{pt}$} \\
\hline Page 6: [594] Formatted & KPachonska & 3/17/20 2:15:00 PM \\
\hline
\end{tabular}

Font: $12 \mathrm{pt}$ 


\begin{tabular}{|c|c|c|}
\hline Page 6: [595] Formatted & KPachonska & 3/17/20 2:15:00 PM \\
\hline \multicolumn{3}{|l|}{ Font: $12 \mathrm{pt}$} \\
\hline Page 6: [596] Formatted & KPachonska & 3/17/20 2:15:00 PM \\
\hline \multicolumn{3}{|l|}{ Font: $12 \mathrm{pt}$} \\
\hline Page 6: [597] Formatted & KPachonska & 3/17/20 2:15:00 PM \\
\hline \multicolumn{3}{|l|}{ Font: $12 \mathrm{pt}$} \\
\hline Page 6: [598] Formatted & KPachonska & 3/17/20 2:15:00 PM \\
\hline \multicolumn{3}{|l|}{ Font: $12 \mathrm{pt}$} \\
\hline Page 6: [599] Formatted & KPachonska & 3/17/20 2:15:00 PM \\
\hline \multicolumn{3}{|l|}{ Font: $12 \mathrm{pt}$} \\
\hline Page 6: [600] Formatted & KPachonska & 3/17/20 2:15:00 PM \\
\hline \multicolumn{3}{|l|}{ Font: $12 \mathrm{pt}$} \\
\hline Page 6: [601] Formatted & KPachonska & 3/17/20 2:15:00 PM \\
\hline \multicolumn{3}{|l|}{ Font: $12 \mathrm{pt}$} \\
\hline Page 6: [602] Formatted & KPachonska & 3/17/20 2:15:00 PM \\
\hline \multicolumn{3}{|c|}{ Normal, Space After: 8 pt, Line spacing: Multiple 1.15 li, Pattern: Clear (White) } \\
\hline Page 6: [603] Formatted & KPachonska & 3/17/20 2:15:00 PM \\
\hline \multicolumn{3}{|l|}{ Font: $12 \mathrm{pt}$} \\
\hline Page 6: [604] Formatted & KPachonska & 3/17/20 2:15:00 PM \\
\hline \multicolumn{3}{|l|}{ Font: $12 \mathrm{pt}$} \\
\hline Page 6: [605] Formatted & KPachonska & 3/17/20 2:15:00 PM \\
\hline \multicolumn{3}{|l|}{ Font: $12 \mathrm{pt}$} \\
\hline Page 6: [606] Formatted & KPachonska & 3/17/20 2:15:00 PM \\
\hline \multicolumn{3}{|l|}{ Font: $12 \mathrm{pt}$} \\
\hline Page 6: [607] Formatted & KPachonska & 3/17/20 2:15:00 PM \\
\hline \multicolumn{3}{|l|}{ Font: $12 \mathrm{pt}$} \\
\hline Page 6: [608] Formatted & KPachonska & 3/17/20 2:15:00 PM \\
\hline \multicolumn{3}{|l|}{ Font: $12 \mathrm{pt}$} \\
\hline Page 6: [609] Formatted & KPachonska & 3/17/20 2:15:00 PM \\
\hline \multicolumn{3}{|l|}{ Font: $12 \mathrm{pt}$} \\
\hline Page 6: [610] Formatted & KPachonska & 3/17/20 2:15:00 PM \\
\hline \multicolumn{3}{|l|}{ Font: $12 \mathrm{pt}$} \\
\hline Page 6: [611] Formatted & KPachonska & 3/17/20 2:15:00 PM \\
\hline \multicolumn{3}{|l|}{ Font: $12 \mathrm{pt}$} \\
\hline Page 6: [612] Formatted & KPachonska & 3/17/20 2:15:00 PM \\
\hline \multicolumn{3}{|l|}{ Font: $12 \mathrm{pt}$} \\
\hline Page 6: [613] Formatted & KPachonska & 3/17/20 2:15:00 PM \\
\hline \multicolumn{3}{|l|}{ Font: $12 \mathrm{pt}$} \\
\hline Page 6: [614] Formatted & KPachonska & 3/17/20 2:15:00 PM \\
\hline
\end{tabular}




\begin{tabular}{|c|c|c|}
\hline Page 6: [615] Formatted & KPachonska & 3/17/20 2:15:00 PM \\
\hline \multicolumn{3}{|l|}{ Font: $12 \mathrm{pt}$} \\
\hline Page 6: [616] Formatted & KPachonska & 3/17/20 2:15:00 PM \\
\hline \multicolumn{3}{|l|}{ Font: $12 \mathrm{pt}$} \\
\hline Page 6: [617] Formatted & KPachonska & 3/17/20 2:15:00 PM \\
\hline \multicolumn{3}{|l|}{ Font: $12 \mathrm{pt}$} \\
\hline Page 6: [617] Formatted & KPachonska & 3/17/20 2:15:00 PM \\
\hline \multicolumn{3}{|l|}{ Font: $12 \mathrm{pt}$} \\
\hline Page 6: [618] Formatted & KPachonska & 3/17/20 2:15:00 PM \\
\hline \multicolumn{3}{|l|}{ Font: $12 \mathrm{pt}$} \\
\hline Page 6: [619] Formatted & KPachonska & 3/17/20 2:15:00 PM \\
\hline \multicolumn{3}{|l|}{ Font: $12 \mathrm{pt}$} \\
\hline Page 6: [620] Formatted & KPachonska & 3/17/20 2:15:00 PM \\
\hline \multicolumn{3}{|l|}{ Font: $12 \mathrm{pt}$} \\
\hline Page 6: [621] Formatted & KPachonska & 3/17/20 2:15:00 PM \\
\hline \multicolumn{3}{|l|}{ Font: $12 \mathrm{pt}$} \\
\hline Page 6: [622] Formatted & KPachonska & 3/17/20 2:15:00 PM \\
\hline \multicolumn{3}{|l|}{ Font: $12 \mathrm{pt}$} \\
\hline Page 6: [623] Formatted & KPachonska & 3/17/20 2:15:00 PM \\
\hline \multicolumn{3}{|l|}{ Font: $12 \mathrm{pt}$} \\
\hline Page 6: [624] Formatted & KPachonska & 3/17/20 2:15:00 PM \\
\hline \multicolumn{3}{|l|}{ Font: $12 \mathrm{pt}$} \\
\hline Page 6: [625] Formatted & KPachonska & 3/17/20 2:15:00 PM \\
\hline \multicolumn{3}{|l|}{ Font: $12 \mathrm{pt}$} \\
\hline Page 6: [626] Formatted & KPachonska & 3/17/20 2:15:00 PM \\
\hline \multicolumn{3}{|l|}{ Font: $12 \mathrm{pt}$} \\
\hline Page 6: [627] Formatted & KPachonska & 3/17/20 2:15:00 PM \\
\hline \multicolumn{3}{|l|}{ Font: $12 \mathrm{pt}$} \\
\hline Page 6: [628] Formatted & KPachonska & 3/17/20 2:15:00 PM \\
\hline \multicolumn{3}{|l|}{ Font: $12 \mathrm{pt}$} \\
\hline Page 6: [628] Formatted & KPachonska & 3/17/20 2:15:00 PM \\
\hline \multicolumn{3}{|l|}{ Font: $12 \mathrm{pt}$} \\
\hline Page 6: [629] Deleted & KPachonska & 3/17/20 2:15:00 PM \\
\hline$\nabla$ & & \\
\hline Page 6: [630] Formatted & KPachonska & 3/17/20 2:15:00 PM \\
\hline \multicolumn{3}{|l|}{ Font: $12 \mathrm{pt}$} \\
\hline Page 6: [631] Formatted & KPachonska & 3/17/20 2:15:00 PM \\
\hline \multicolumn{3}{|l|}{ Font: $12 \mathrm{pt}$} \\
\hline Page 6: [632] Formatted & KPachonska & 3/17/20 2:15:00 PM \\
\hline
\end{tabular}


Font: $12 \mathrm{pt}$

\begin{tabular}{|c|c|c|}
\hline Page 6: [633] Formatted & KPachonska & 3/17/20 2:15:00 PM \\
\hline \multicolumn{3}{|l|}{ Font: $12 \mathrm{pt}$} \\
\hline Page 6: [634] Formatted & KPachonska & 3/17/20 2:15:00 PM \\
\hline \multicolumn{3}{|l|}{ Font: $12 \mathrm{pt}$} \\
\hline Page 1: [635] Formatted & KPachonska & 3/17/20 2:15:00 PM \\
\hline \multicolumn{3}{|c|}{ Normal, Tab stops: $6.26 "$, Right } \\
\hline Page 1: [636] Formatted & KPachonska & 3/17/20 2:15:00 PM \\
\hline \multicolumn{3}{|c|}{ Normal, Tab stops: $6.26 "$ ", Right } \\
\hline Page 7: [637] Formatted & KPachonska & 3/17/20 2:15:00 PM \\
\hline \multicolumn{3}{|l|}{ Font: $12 \mathrm{pt}$} \\
\hline Page 7: [638] Deleted & KPachonska & 3/17/20 2:15:00 PM \\
\hline \multicolumn{3}{|l|}{ 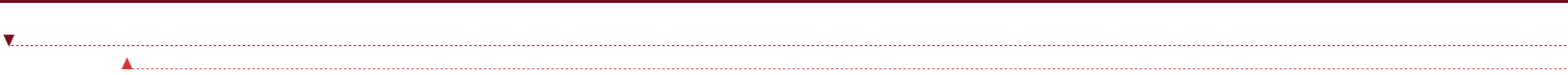 } \\
\hline Page 7: [639] Deleted & KPachonska & 3/17/20 2:15:00 PM \\
\hline Page 7: [640] Formatted & KPachonska & 3/17/20 2:15:00 PM \\
\hline \multicolumn{3}{|l|}{ Font: $12 \mathrm{pt}$} \\
\hline Page 7: [641] Deleted & KPachonska & 3/17/20 2:15:00 PM \\
\hline$\checkmark$ & & \\
\hline Page 7: [642] Formatted & KPachonska & 3/17/20 2:15:00 PM \\
\hline \multicolumn{3}{|l|}{ Font: $12 \mathrm{pt}$} \\
\hline Page 7: [643] Deleted & KPachonska & 3/17/20 2:15:00 PM \\
\hline & & \\
\hline Page 7: [644] Formatted & KPachonska & 3/17/20 2:15:00 PM \\
\hline \multicolumn{3}{|l|}{ Font: $12 \mathrm{pt}$} \\
\hline Page 7: [645] Formatted & KPachonska & 3/17/20 2:15:00 PM \\
\hline \multicolumn{3}{|l|}{ Font: $12 \mathrm{pt}$} \\
\hline Page 7: [645] Formatted & KPachonska & 3/17/20 2:15:00 PM \\
\hline \multicolumn{3}{|l|}{ Font: $12 \mathrm{pt}$} \\
\hline Page 7: [646] Formatted & KPachonska & 3/17/20 2:15:00 PM \\
\hline \multicolumn{3}{|l|}{ Font: $12 \mathrm{pt}$} \\
\hline Page 7: [647] Formatted & KPachonska & 3/17/20 2:15:00 PM \\
\hline \multicolumn{3}{|l|}{ Font: $12 \mathrm{pt}$} \\
\hline Page 7: [648] Formatted & KPachonska & 3/17/20 2:15:00 PM \\
\hline \multicolumn{3}{|l|}{ Font: $12 \mathrm{pt}$} \\
\hline Page 7: [649] Formatted & KPachonska & 3/17/20 2:15:00 PM \\
\hline \multicolumn{3}{|l|}{ Font: $12 \mathrm{pt}$} \\
\hline Page 7: [649] Formatted & KPachonska & 3/17/20 2:15:00 PM \\
\hline
\end{tabular}




\begin{tabular}{|c|c|c|}
\hline Page 7: [650] Formatted & KPachonska & 3/17/20 2:15:00 PM \\
\hline \multicolumn{3}{|l|}{ Font: $12 \mathrm{pt}$} \\
\hline Page 7: [651] Deleted & KPachonska & 3/17/20 2:15:00 PM \\
\hline Pave 7. 66521 Formatted & KPachonska & 3/17/20 2:15:00 PM \\
\hline \multicolumn{3}{|l|}{ Formatted } \\
\hline Page 7: [653] Formatted & KPachonska & 3/17/20 2:15:00 PM \\
\hline \multicolumn{3}{|l|}{ Formatted } \\
\hline Page 7: [654] Formatted & KPachonska & 3/17/20 2:15:00 PM \\
\hline \multicolumn{3}{|l|}{ Formatted } \\
\hline Page 7: [655] Formatted & KPachonska & 3/17/20 2:15:00 PM \\
\hline \multicolumn{3}{|l|}{ Formatted } \\
\hline Page 7: [656] Formatted & KPachonska & 3/17/20 2:15:00 PM \\
\hline \multicolumn{3}{|l|}{ Formatted } \\
\hline Page 7: [657] Formatted & KPachonska & 3/17/20 2:15:00 PM \\
\hline \multicolumn{3}{|l|}{ Formatted } \\
\hline Page 7: [658] Deleted & KPachonska & 3/17/20 2:15:00 PM \\
\hline \multicolumn{3}{|l|}{$\nabla$} \\
\hline \multicolumn{3}{|l|}{$\Delta$} \\
\hline Page 7: [659] Formatted & KPachonska & 3/17/20 2:15:00 PM \\
\hline \multicolumn{3}{|l|}{ Font: $12 \mathrm{pt}$} \\
\hline Page 7: [660] Formatted & KPachonska & 3/17/20 2:15:00 PM \\
\hline \multicolumn{3}{|c|}{ Normal, Space After: 8 pt, Line spacing: Multiple 1.15 li, Pattern: Clear (White) } \\
\hline Page 7: [661] Formatted & KPachonska & 3/17/20 2:15:00 PM \\
\hline \multicolumn{3}{|l|}{ Font: $12 \mathrm{pt}$} \\
\hline Page 7: [662] Formatted & KPachonska & 3/17/20 2:15:00 PM \\
\hline \multicolumn{3}{|l|}{ Font: $12 \mathrm{pt}$} \\
\hline Page 7: [663] Formatted & KPachonska & 3/17/20 2:15:00 PM \\
\hline \multicolumn{3}{|l|}{ Font: $12 \mathrm{pt}$} \\
\hline Page 7: [664] Formatted & KPachonska & 3/17/20 2:15:00 PM \\
\hline \multicolumn{3}{|l|}{ Font: $12 \mathrm{pt}$} \\
\hline Page 7: [665] Formatted & KPachonska & 3/17/20 2:15:00 PM \\
\hline \multicolumn{3}{|l|}{ Font: $12 \mathrm{pt}$} \\
\hline Page 7: [666] Formatted & KPachonska & 3/17/20 2:15:00 PM \\
\hline \multicolumn{3}{|l|}{ Font: $12 \mathrm{pt}$} \\
\hline Page 7: [667] Formatted & KPachonska & 3/17/20 2:15:00 PM \\
\hline \multicolumn{3}{|l|}{ Font: $12 \mathrm{pt}$} \\
\hline Page 7: [668] Formatted & KPachonska & 3/17/20 2:15:00 PM \\
\hline \multicolumn{3}{|l|}{ Font: $12 \mathrm{pt}$} \\
\hline Page 7: [669] Formatted & KPachonska & 3/17/20 2:15:00 PM \\
\hline
\end{tabular}


Font: $12 \mathrm{pt}$

Page 7: [670] Formatted

KPachonska

3/17/20 2:15:00 PM

Font: $12 \mathrm{pt}$

Page 7: [671] Formatted

KPachonska

3/17/20 2:15:00 PM

Font: $12 \mathrm{pt}$

Page 7: [672] Formatted

KPachonska

3/17/20 2:15:00 PM

Font: $12 \mathrm{pt}$

Page 7: [673] Formatted

KPachonska

3/17/20 2:15:00 PM

Font: $12 \mathrm{pt}$

Page 7: [674] Formatted

KPachonska

3/17/20 2:15:00 PM

Formatted

Page 7: [674] Formatted

KPachonska

3/17/20 2:15:00 PM

Formatted

Page 7: [675] Commented [JMS3]

Julia Massimelli Sewall

3/24/20 9:19:00 PM

be consistent. Either call groups junior, senior, or year.

\begin{tabular}{|c|c|c|}
\hline Page 7: [676] Formatted & KPachonska & 3/17/20 2:15:00 PM \\
\hline \multicolumn{3}{|l|}{ Formatted } \\
\hline Page 7: [677] Deleted & KPachonska & 3/17/20 2:15:00 PM \\
\hline Page $7:[678]$ Formatted & KPachonska & 3/17/20 2:15:00 PM \\
\hline \multicolumn{3}{|l|}{ Font: $12 \mathrm{pt}$} \\
\hline Page 7: [678] Formatted & KPachonska & 3/17/20 2:15:00 PM \\
\hline \multicolumn{3}{|l|}{ Font: $12 \mathrm{pt}$} \\
\hline Page 7: [679] Formatted & KPachonska & 3/17/20 2:15:00 PM \\
\hline \multicolumn{3}{|l|}{ Font: $12 \mathrm{pt}$} \\
\hline Page 7: [680] Formatted & KPachonska & 3/17/20 2:15:00 PM \\
\hline \multicolumn{3}{|l|}{ Font: $12 \mathrm{pt}$} \\
\hline Page 7: [681] Formatted & KPachonska & 3/17/20 2:15:00 PM \\
\hline \multicolumn{3}{|l|}{ Font: $12 \mathrm{pt}$} \\
\hline Page 7: [682] Formatted & KPachonska & 3/17/20 2:15:00 PM \\
\hline \multicolumn{3}{|l|}{ Font: $12 \mathrm{pt}$} \\
\hline Page 7: [683] Formatted & KPachonska & 3/17/20 2:15:00 PM \\
\hline \multicolumn{3}{|l|}{ Font: $12 \mathrm{pt}$} \\
\hline Page 7: [684] Formatted & KPachonska & 3/17/20 2:15:00 PM \\
\hline \multicolumn{3}{|l|}{ Font: Bold } \\
\hline Page 7: [685] Formatted & KPachonska & 3/17/20 2:15:00 PM \\
\hline \multicolumn{3}{|l|}{ Font: $12 \mathrm{pt}$, Bold } \\
\hline Page 7: [686] Deleted & KPachonska & 3/17/20 2:15:00 PM \\
\hline 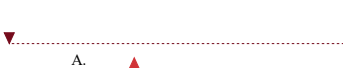 & & \\
\hline Page 7: [687] Formatted & KPachonska & 3/17/20 2:15:00 PM \\
\hline
\end{tabular}


Font: Bold

\begin{tabular}{|c|c|c|}
\hline Page 7: [689] Formatted & KPachonska & 3/17/20 2:15:00 PM \\
\hline \multicolumn{3}{|l|}{ Font: $12 \mathrm{pt}$} \\
\hline Page 7: [690] Formatted & KPachonska & 3/17/20 2:15:00 PM \\
\hline \multicolumn{3}{|l|}{ Font: $12 \mathrm{pt}$} \\
\hline Page 7: [691] Formatted & KPachonska & 3/17/20 2:15:00 PM \\
\hline \multicolumn{3}{|l|}{ Font: $12 \mathrm{pt}$} \\
\hline Page 7: [692] Formatted & KPachonska & 3/17/20 2:15:00 PM \\
\hline \multicolumn{3}{|l|}{ Font: $12 \mathrm{pt}$} \\
\hline Page 7: [693] Formatted & KPachonska & 3/17/20 2:15:00 PM \\
\hline \multicolumn{3}{|l|}{ Font: $12 \mathrm{pt}$} \\
\hline Page 7: [694] Formatted & KPachonska & 3/17/20 2:15:00 PM \\
\hline \multicolumn{3}{|l|}{ Font: $12 \mathrm{pt}$} \\
\hline Page 7: [695] Formatted & KPachonska & 3/17/20 2:15:00 PM \\
\hline \multicolumn{3}{|l|}{ Font: $12 \mathrm{pt}$} \\
\hline Page 7: [696] Formatted & KPachonska & 3/17/20 2:15:00 PM \\
\hline \multicolumn{3}{|l|}{ Font: $12 \mathrm{pt}$} \\
\hline Page 7: [697] Formatted & KPachonska & 3/17/20 2:15:00 PM \\
\hline \multicolumn{3}{|l|}{ Font: $12 \mathrm{pt}$} \\
\hline Page 7: [698] Deleted & KPachonska & 3/17/20 2:15:00 PM \\
\hline$\checkmark$ & & \\
\hline Page 7: [699] Formatted & KPachonska & 3/17/20 2:15:00 PM \\
\hline \multicolumn{3}{|l|}{ Font: $12 \mathrm{pt}$} \\
\hline Page 7: $[700]$ Formatted & KPachonska & 3/17/20 2:15:00 PM \\
\hline \multicolumn{3}{|l|}{ Font: $12 \mathrm{pt}$} \\
\hline Page 7: [701] Formatted & KPachonska & 3/17/20 2:15:00 PM \\
\hline \multicolumn{3}{|l|}{ Font: $12 \mathrm{pt}$} \\
\hline Page 7: [702] Formatted & KPachonska & 3/17/20 2:15:00 PM \\
\hline \multicolumn{3}{|l|}{ Font: $12 \mathrm{pt}$} \\
\hline Page 7: [703] Formatted & KPachonska & 3/17/20 2:15:00 PM \\
\hline \multicolumn{3}{|l|}{ Font: $12 \mathrm{pt}$} \\
\hline Page 7: [704] Formatted & KPachonska & 3/17/20 2:15:00 PM \\
\hline \multicolumn{3}{|l|}{ Font: $12 \mathrm{pt}$} \\
\hline Page 7: [705] Formatted & KPachonska & 3/17/20 2:15:00 PM \\
\hline
\end{tabular}


Font: $12 \mathrm{pt}$

Page 7: [706] Formatted

KPachonska

3/17/20 2:15:00 PM

Font: $12 \mathrm{pt}$

Page 7: [707] Formatted

KPachonska

3/17/20 2:15:00 PM

Font: $12 \mathrm{pt}$

Page 7: [708] Formatted

KPachonska

3/17/20 2:15:00 PM

Font: $12 \mathrm{pt}$

Page 7: [709] Formatted

KPachonska

3/17/20 2:15:00 PM

Font: $12 \mathrm{pt}$

Page 7: [710] Formatted

KPachonska

3/17/20 2:15:00 PM

Font: $12 \mathrm{pt}$

Page 7: [711] Formatted

KPachonska

3/17/20 2:15:00 PM

Font: $12 \mathrm{pt}$

Page 7: [712] Formatted

KPachonska

3/17/20 2:15:00 PM

Font: $12 \mathrm{pt}$

Page 7: [713] Formatted

KPachonska

3/17/20 2:15:00 PM

Font: $12 \mathrm{pt}$

Page 7: [714] Formatted

KPachonska

3/17/20 2:15:00 PM

Font: $12 \mathrm{pt}$

Page 7: [715] Formatted

KPachonska

3/17/20 2:15:00 PM

Font: $12 \mathrm{pt}$

Page 7: [716] Formatted

KPachonska

3/17/20 2:15:00 PM

Font: $12 \mathrm{pt}$

Page 7: [717] Formatted

KPachonska

3/17/20 2:15:00 PM

Font: $12 \mathrm{pt}$

Page 7: [718] Formatted

KPachonska

3/17/20 2:15:00 PM

Font: $12 \mathrm{pt}$

Page 7: [719] Deleted

KPachonska

3/17/20 2:15:00 PM

$\checkmark$

Page 1: [720] Formatted

KPachonska

3/17/20 2:15:00 PM

Normal, Tab stops: 6.26", Right

Page 1: [721] Formatted

KPachonska

3/17/20 2:15:00 PM

Normal, Tab stops: 6.26", Right

Page 8: [722] Formatted

KPachonska

3/17/20 2:15:00 PM

Normal, Indent: First line: 0.5", Space After: 8 pt, Line spacing: Multiple 1.15 li, Pattern: Clear (White)

Page 8: [723] Deleted

KPachonska

3/17/20 2:15:00 PM

Page 8: [723] Deleted

KPachonska

3/17/20 2:15:00 PM 


\begin{tabular}{|c|c|c|}
\hline Page 8: [723] Deleted & KPachonska & 3/17/20 2:15:00 PM \\
\hline$\nabla$ & & \\
\hline Page 8: [723] Deleted & KPachonska & 3/17/20 2:15:00 PM \\
\hline$\nabla$. & & \\
\hline Page 8: [723] Deleted & KPachonska & 3/17/20 2:15:00 PM \\
\hline$\checkmark \ldots$ & & \\
\hline Page 8: [723] Deleted & KPachonska & 3/17/20 2:15:00 PM \\
\hline$\nabla$ & & \\
\hline Page 8: [724] Formatted & KPachonska & 3/17/20 2:15:00 PM \\
\hline \multicolumn{3}{|l|}{ Font: $12 \mathrm{pt}$} \\
\hline Page 8: [725] Deleted & KPachonska & 3/17/20 2:15:00 PM \\
\hline$\checkmark$ & & \\
\hline \multicolumn{3}{|l|}{$\boldsymbol{\Lambda}$} \\
\hline Page 8: [725] Deleted & KPachonska & 3/17/20 2:15:00 PM \\
\hline \multicolumn{3}{|l|}{$\Delta$} \\
\hline Page 8: [726] Formatted & KPachonska & 3/17/20 2:15:00 PM \\
\hline \multicolumn{3}{|l|}{ Font: $12 \mathrm{pt}$} \\
\hline Page 8: [726] Formatted & KPachonska & 3/17/20 2:15:00 PM \\
\hline \multicolumn{3}{|l|}{ Font: $12 \mathrm{pt}$} \\
\hline Page 8: [727] Formatted & KPachonska & 3/17/20 2:15:00 PM \\
\hline \multicolumn{3}{|l|}{ Font: $12 \mathrm{pt}$} \\
\hline Page 8: [728] Deleted & KPachonska & 3/17/20 2:15:00 PM \\
\hline$\nabla$. & & \\
\hline Page 8: [729] Formatted & KPachonska & 3/17/20 2:15:00 PM \\
\hline \multicolumn{3}{|l|}{ Font: $12 \mathrm{pt}$} \\
\hline Page 8: [730] Deleted & KPachonska & 3/17/20 2:15:00 PM \\
\hline$\checkmark \ldots \ldots$ & & \\
\hline Page 8: [731] Formatted & KPachonska & 3/17/20 2:15:00 PM \\
\hline \multicolumn{3}{|l|}{ Font: $12 \mathrm{pt}$} \\
\hline Page 8: [732] Formatted & KPachonska & 3/17/20 2:15:00 PM \\
\hline \multicolumn{3}{|c|}{ Normal, Space After: 8 pt, Line spacing: Multiple 1.15 li, Pattern: Clear (White) } \\
\hline Page 8: [733] Formatted & KPachonska & 3/17/20 2:15:00 PM \\
\hline \multicolumn{3}{|l|}{ Font: $12 \mathrm{pt}$} \\
\hline Page 8: [734] Formatted & KPachonska & 3/17/20 2:15:00 PM \\
\hline
\end{tabular}

Font: 12 pt 


\begin{tabular}{|c|c|c|}
\hline Page 8: [734] Formatted & KPachonska & 3/17/20 2:15:00 PM \\
\hline \multicolumn{3}{|l|}{ Font: $12 \mathrm{pt}$} \\
\hline Page 8: [735] Formatted & KPachonska & 3/17/20 2:15:00 PM \\
\hline \multicolumn{3}{|l|}{ Font: $12 \mathrm{pt}$} \\
\hline Page 8: [736] Formatted & KPachonska & 3/17/20 2:15:00 PM \\
\hline \multicolumn{3}{|l|}{ Font: $12 \mathrm{pt}$} \\
\hline Page 8: [737] Formatted & KPachonska & 3/17/20 2:15:00 PM \\
\hline \multicolumn{3}{|l|}{ Font: $12 \mathrm{pt}$} \\
\hline Page 8: [738] Formatted & KPachonska & 3/17/20 2:15:00 PM \\
\hline \multicolumn{3}{|l|}{ Font: $12 \mathrm{pt}$} \\
\hline Page 8: [739] Formatted & KPachonska & 3/17/20 2:15:00 PM \\
\hline \multicolumn{3}{|l|}{ Font: $12 \mathrm{pt}$} \\
\hline Page 8: [740] Formatted & KPachonska & 3/17/20 2:15:00 PM \\
\hline \multicolumn{3}{|l|}{ Font: $12 \mathrm{pt}$} \\
\hline Page 8: [741] Formatted & KPachonska & 3/17/20 2:15:00 PM \\
\hline \multicolumn{3}{|l|}{ Font: $12 \mathrm{pt}$} \\
\hline Page 8: [742] Formatted & KPachonska & 3/17/20 2:15:00 PM \\
\hline \multicolumn{3}{|l|}{ Font: $12 \mathrm{pt}$} \\
\hline Page 8: [743] Commented [JMS4] & Julia Massimelli Sewall & 3/24/20 9:23:00 PM \\
\hline \multicolumn{3}{|c|}{ Add context, what are you trying to say other that point out SD } \\
\hline Page 8: [744] Deleted & KPachonska & 3/17/20 2:15:00 PM \\
\hline \multirow{2}{*}{\multicolumn{3}{|c|}{$\begin{array}{l}\text { Page 8: [745] Formatted } \\
\text { Font: } 12 \mathrm{pt}\end{array}$}} \\
\hline & & \\
\hline Page 8: [746] Formatted & KPachonska & 3/17/20 2:15:00 PM \\
\hline \multicolumn{3}{|c|}{ Normal, Space After: 8 pt, Line spacing: Multiple 1.15 li, Pattern: Clear (White) } \\
\hline Page 8: [747] Formatted & KPachonska & 3/17/20 2:15:00 PM \\
\hline \multicolumn{3}{|l|}{ Font: $12 \mathrm{pt}$} \\
\hline Page 8: [748] Formatted & KPachonska & 3/17/20 2:15:00 PM \\
\hline \multicolumn{3}{|l|}{ Font: $12 \mathrm{pt}$} \\
\hline Page 8: [748] Formatted & KPachonska & 3/17/20 2:15:00 PM \\
\hline \multicolumn{3}{|l|}{ Font: $12 \mathrm{pt}$} \\
\hline Page 8: [748] Formatted & KPachonska & 3/17/20 2:15:00 PM \\
\hline \multicolumn{3}{|l|}{ Font: $12 \mathrm{pt}$} \\
\hline Page 8: [748] Formatted & KPachonska & 3/17/20 2:15:00 PM \\
\hline \multicolumn{3}{|l|}{ Font: $12 \mathrm{pt}$} \\
\hline Page 8: [748] Formatted & KPachonska & 3/17/20 2:15:00 PM \\
\hline \multicolumn{3}{|l|}{ Font: $12 \mathrm{pt}$} \\
\hline Page 8: [748] Formatted & KPachonska & 3/17/20 2:15:00 PM \\
\hline \multicolumn{3}{|l|}{ Font: $12 \mathrm{pt}$} \\
\hline Page 8: [748] Formatted & KPachonska & 3/17/20 2:15:00 PM \\
\hline
\end{tabular}


Font: $12 \mathrm{pt}$

Page 8: [749] Formatted

KPachonska

3/17/20 2:15:00 PM

Font: $12 \mathrm{pt}$

Page 8: [749] Formatted

KPachonska

3/17/20 2:15:00 PM

Font: $12 \mathrm{pt}$

Page 8: [749] Formatted

KPachonska

3/17/20 2:15:00 PM

Font: $12 \mathrm{pt}$

Page 8: [750] Formatted

KPachonska

3/17/20 2:15:00 PM

Font: $12 \mathrm{pt}$

Page 8: [751] Formatted

KPachonska

3/17/20 2:15:00 PM

Font: $12 \mathrm{pt}$

Page 8: [751] Formatted

KPachonska

3/17/20 2:15:00 PM

Font: $12 \mathrm{pt}$

Page 8: [752] Formatted

KPachonska

3/17/20 2:15:00 PM

Font: Bold

Page 8: [753] Deleted

KPachonska

3/17/20 2:15:00 PM

$\checkmark$

Page 8: [754] Formatted

KPachonska

3/17/20 2:15:00 PM

Font: $12 \mathrm{pt}$

Page 8: [755] Formatted

KPachonska

3/17/20 2:15:00 PM

Font: $12 \mathrm{pt}$

Page 8: [756] Formatted

KPachonska

3/17/20 2:15:00 PM

Font: $12 \mathrm{pt}$

Page 8: [757] Formatted

KPachonska

3/17/20 2:15:00 PM

Font: $12 \mathrm{pt}$

Page 8: [758] Formatted

KPachonska

3/17/20 2:15:00 PM

Font: $12 \mathrm{pt}$

Page 8: [759] Formatted

KPachonska

3/17/20 2:15:00 PM

Font: $12 \mathrm{pt}$

Page 8: [760] Formatted

KPachonska

3/17/20 2:15:00 PM

Font: $12 \mathrm{pt}$

Page 8: [761] Formatted

KPachonska

3/17/20 2:15:00 PM

Font: $12 \mathrm{pt}$

Page 8: [762] Formatted

KPachonska

3/17/20 2:15:00 PM

Font: $12 \mathrm{pt}$

Page 8: [763] Formatted

KPachonska

3/17/20 2:15:00 PM

Font: $12 \mathrm{pt}$

Page 8: [764] Deleted

KPachonska

3/17/20 2:15:00 PM 


\begin{tabular}{|c|c|c|}
\hline Page 8: [765] Formatted & KPachonska & 3/17/20 2:15:00 PM \\
\hline \multicolumn{3}{|l|}{ Font: $12 \mathrm{pt}$} \\
\hline Page 8: $[766]$ Formatted & KPachonska & 3/17/20 2:15:00 PM \\
\hline \multicolumn{3}{|l|}{ Font: $12 \mathrm{pt}$} \\
\hline Page 8: [767] Formatted & KPachonska & 3/17/20 2:15:00 PM \\
\hline \multicolumn{3}{|l|}{ Font: $12 \mathrm{pt}$} \\
\hline Page 8: [768] Formatted & KPachonska & 3/17/20 2:15:00 PM \\
\hline \multicolumn{3}{|l|}{ Font: $12 \mathrm{pt}$} \\
\hline Page 8: [769] Formatted & KPachonska & 3/17/20 2:15:00 PM \\
\hline \multicolumn{3}{|l|}{ Font: $12 \mathrm{pt}$} \\
\hline Page 8: [770] Formatted & KPachonska & 3/17/20 2:15:00 PM \\
\hline \multicolumn{3}{|l|}{ Font: $12 \mathrm{pt}$} \\
\hline Page 8: [771] Formatted & KPachonska & 3/17/20 2:15:00 PM \\
\hline \multicolumn{3}{|l|}{ Font: $12 \mathrm{pt}$} \\
\hline Page 8: $[772]$ Formatted & KPachonska & 3/17/20 2:15:00 PM \\
\hline \multicolumn{3}{|l|}{ Font: $12 \mathrm{pt}$} \\
\hline Page 8: [773] Deleted & KPachonska & 3/17/20 2:15:00 PM \\
\hline \multicolumn{3}{|l|}{$\checkmark \ldots$} \\
\hline Page 8: [774] Formatted & KPachonska & 3/17/20 2:15:00 PM \\
\hline \multicolumn{3}{|l|}{ Formatted } \\
\hline Page 8: [775] Formatted & KPachonska & 3/17/20 2:15:00 PM \\
\hline \multicolumn{3}{|l|}{ Formatted } \\
\hline Page 8: [775] Formatted & KPachonska & 3/17/20 2:15:00 PM \\
\hline \multicolumn{3}{|l|}{ Formatted } \\
\hline Page 8: [775] Formatted & KPachonska & 3/17/20 2:15:00 PM \\
\hline \multicolumn{3}{|l|}{ Formatted } \\
\hline Page 8: [776] Formatted & KPachonska & 3/17/20 2:15:00 PM \\
\hline \multicolumn{3}{|l|}{ Formatted } \\
\hline Page 8: [777] Formatted & KPachonska & 3/17/20 2:15:00 PM \\
\hline \multicolumn{3}{|l|}{ Formatted } \\
\hline Page 8: [778] Formatted & KPachonska & 3/17/20 2:15:00 PM \\
\hline \multicolumn{3}{|l|}{ Formatted } \\
\hline Page 8: [779] Deleted & KPachonska & 3/17/20 2:15:00 PM \\
\hline \multicolumn{3}{|l|}{$+\ldots \ldots \ldots \ldots \ldots \ldots$} \\
\hline Page 1: $[780]$ Formatted & KPachonska & 3/17/20 2:15:00 PM \\
\hline \multicolumn{3}{|c|}{ Normal, Tab stops: 6.26", Right } \\
\hline Page 1: [781] Formatted & KPachonska & 3/17/20 2:15:00 PM \\
\hline
\end{tabular}

Normal, Tab stops: 6.26", Right 


\begin{tabular}{|c|c|c|}
\hline Page 9: [782] Deleted & KPachonska & 3/17/20 2:15:00 PM \\
\hline$\checkmark$ & & \\
\hline Page 9: [783] Formatted & KPachonska & 3/17/20 2:15:00 PM \\
\hline \multicolumn{3}{|l|}{ Font: $12 \mathrm{pt}$} \\
\hline Page 9: [784] Formatted & KPachonska & 3/17/20 2:15:00 PM \\
\hline \multicolumn{3}{|l|}{ Font: $12 \mathrm{pt}$} \\
\hline Page 9: [785] Formatted & KPachonska & 3/17/20 2:15:00 PM \\
\hline \multicolumn{3}{|l|}{ Font: $12 \mathrm{pt}$} \\
\hline Page 9: [786] Formatted & KPachonska & 3/17/20 2:15:00 PM \\
\hline \multicolumn{3}{|l|}{ Font: $12 \mathrm{pt}$} \\
\hline Page 9: [787] Formatted & KPachonska & 3/17/20 2:15:00 PM \\
\hline \multicolumn{3}{|l|}{ Font: $12 \mathrm{pt}$} \\
\hline Page 9: [788] Deleted & KPachonska & 3/17/20 2:15:00 PM \\
\hline \multicolumn{3}{|l|}{$\nabla+\cdots$} \\
\hline Page 9: [789] Formatted & KPachonska & 3/17/20 2:15:00 PM \\
\hline \multicolumn{3}{|l|}{ Font: $12 \mathrm{pt}$} \\
\hline Page 9: $[790]$ Formatted & KPachonska & 3/17/20 2:15:00 PM \\
\hline \multicolumn{3}{|l|}{ Font: $12 \mathrm{pt}$} \\
\hline Page 9: [791] Formatted & KPachonska & 3/17/20 2:15:00 PM \\
\hline \multicolumn{3}{|l|}{ Font: $12 \mathrm{pt}$} \\
\hline Page 9: [792] Formatted & KPachonska & 3/17/20 2:15:00 PM \\
\hline \multicolumn{3}{|l|}{ Font: $12 \mathrm{pt}$} \\
\hline Page 9: [793] Formatted & KPachonska & 3/17/20 2:15:00 PM \\
\hline \multicolumn{3}{|l|}{ Font: $12 \mathrm{pt}$} \\
\hline Page 9: [794] Formatted & KPachonska & 3/17/20 2:15:00 PM \\
\hline \multicolumn{3}{|c|}{ Normal, Space After: 8 pt, Line spacing: Multiple 1.15 li, Pattern: Clear (White) } \\
\hline Page 9: [795] Formatted & KPachonska & 3/17/20 2:15:00 PM \\
\hline \multicolumn{3}{|l|}{ Font: $12 \mathrm{pt}$} \\
\hline Page 9: [796] Formatted & KPachonska & 3/17/20 2:15:00 PM \\
\hline \multicolumn{3}{|l|}{ Font: $12 \mathrm{pt}$} \\
\hline Page 9: [796] Formatted & KPachonska & 3/17/20 2:15:00 PM \\
\hline \multicolumn{3}{|l|}{ Font: $12 \mathrm{pt}$} \\
\hline Page 9: [797] Formatted & KPachonska & 3/17/20 2:15:00 PM \\
\hline \multicolumn{3}{|l|}{ Font: $12 \mathrm{pt}$} \\
\hline Page 9: [798] Formatted & KPachonska & 3/17/20 2:15:00 PM \\
\hline \multicolumn{3}{|l|}{ Font: $12 \mathrm{pt}$} \\
\hline Page 9: [799] Formatted & KPachonska & 3/17/20 2:15:00 PM \\
\hline \multicolumn{3}{|l|}{ Font: $12 \mathrm{pt}$} \\
\hline Page 9: [800] Formatted & KPachonska & 3/17/20 2:15:00 PM \\
\hline
\end{tabular}


Font: $12 \mathrm{pt}$

Page 9: [800] Formatted

KPachonska

3/17/20 2:15:00 PM

Font: $12 \mathrm{pt}$

Page 9: [801] Formatted

KPachonska

3/17/20 2:15:00 PM

Font: $12 \mathrm{pt}$

Page 9: [802] Formatted

KPachonska

3/17/20 2:15:00 PM

Font: $12 \mathrm{pt}$

Page 9: [803] Formatted

KPachonska

3/17/20 2:15:00 PM

Font: $12 \mathrm{pt}$

Page 9: [804] Deleted

KPachonska

3/17/20 2:15:00 PM

\begin{tabular}{|c|c|c|}
\hline Page 9: [805] Formatted & KPachonska & 3/17/20 2:15:00 PM \\
\hline \multicolumn{3}{|l|}{ Font: $12 \mathrm{pt}$} \\
\hline Page 9: [806] Formatted & KPachonska & 3/17/20 2:15:00 PM \\
\hline \multicolumn{3}{|l|}{ Font: $12 \mathrm{pt}$} \\
\hline Page 9: [807] Formatted & KPachonska & 3/17/20 2:15:00 PM \\
\hline
\end{tabular}

Normal, Space After: 8 pt, Line spacing: Multiple 1.15 li, Pattern: Clear (White)

\begin{tabular}{|c|c|c|}
\hline Page 9: [808] Formatted & KPachonska & 3/17/20 2:15:00 PM \\
\hline \multicolumn{3}{|l|}{ Font: $12 \mathrm{pt}$} \\
\hline Page 9: [809] Formatted & KPachonska & 3/17/20 2:15:00 PM \\
\hline \multicolumn{3}{|l|}{ Font: $12 \mathrm{pt}$} \\
\hline Page 9: [809] Formatted & KPachonska & 3/17/20 2:15:00 PM \\
\hline \multicolumn{3}{|l|}{ Font: $12 \mathrm{pt}$} \\
\hline Page 9: [810] Formatted & KPachonska & 3/17/20 2:15:00 PM \\
\hline \multicolumn{3}{|l|}{ Font: $12 \mathrm{pt}$} \\
\hline Page 9: [811] Formatted & KPachonska & 3/17/20 2:15:00 PM \\
\hline \multicolumn{3}{|l|}{ Font: $12 \mathrm{pt}$} \\
\hline Page 9: [812] Deleted & KPachonska & 3/17/20 2:15:00 PM \\
\hline \multicolumn{3}{|l|}{$\boldsymbol{\nabla}$} \\
\hline Page 9: [813] Formatted & KPachonska & 3/17/20 2:15:00 PM \\
\hline \multicolumn{3}{|l|}{ Font: $12 \mathrm{pt}$} \\
\hline Page 9: [814] Deleted & KPachonska & 3/17/20 2:15:00 PM \\
\hline \multicolumn{3}{|l|}{ 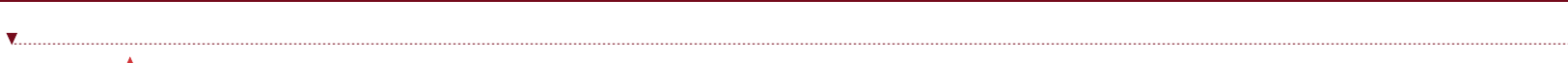 } \\
\hline Page 9: [815] Formatted & KPachonska & 3/17/20 2:15:00 PM \\
\hline \multicolumn{3}{|l|}{ Font: $12 \mathrm{pt}$} \\
\hline Page 9: [816] Deleted & KPachonska & 3/17/20 2:15:00 PM \\
\hline
\end{tabular}




\begin{tabular}{|c|c|c|}
\hline Page 9: [817] Formatted & KPachonska & 3/17/20 2:15:00 PM \\
\hline \multicolumn{3}{|l|}{ Font: $12 \mathrm{pt}$} \\
\hline Page 9: [818] Deleted & KPachonska & 3/17/20 2:15:00 PM \\
\hline$v$ & & \\
\hline Page 9: [819] Formatted & KPachonska & 3/17/20 2:15:00 PM \\
\hline \multicolumn{3}{|c|}{ Font: 12 pt, Font color: Black } \\
\hline Page 9: [820] Formatted & KPachonska & 3/17/20 2:15:00 PM \\
\hline \multicolumn{3}{|c|}{ Font: 12 pt, Font color: Black } \\
\hline Page 9: [821] Formatted & KPachonska & 3/17/20 2:15:00 PM \\
\hline \multicolumn{3}{|l|}{ Formatted } \\
\hline Page 9: [822] Formatted & KPachonska & 3/17/20 2:15:00 PM \\
\hline \multicolumn{3}{|l|}{ Font: $12 \mathrm{pt}$} \\
\hline Page 9: [823] Formatted & KPachonska & 3/17/20 2:15:00 PM \\
\hline \multicolumn{3}{|l|}{ Font: $12 \mathrm{pt}$} \\
\hline Page 9: [824] Formatted & KPachonska & 3/17/20 2:15:00 PM \\
\hline \multicolumn{3}{|l|}{ Font: $12 \mathrm{pt}$} \\
\hline Page 9: [825] Formatted & KPachonska & 3/17/20 2:15:00 PM \\
\hline \multicolumn{3}{|l|}{ Font: $12 \mathrm{pt}$} \\
\hline Page 9: [826] Formatted & KPachonska & 3/17/20 2:15:00 PM \\
\hline \multicolumn{3}{|l|}{ Font: $12 \mathrm{pt}$} \\
\hline Page 9: [826] Formatted & KPachonska & 3/17/20 2:15:00 PM \\
\hline \multicolumn{3}{|l|}{ Font: $12 \mathrm{pt}$} \\
\hline Page 9: [827] Formatted & KPachonska & 3/17/20 2:15:00 PM \\
\hline \multicolumn{3}{|l|}{ Font: $12 \mathrm{pt}$} \\
\hline Page 9: [828] Formatted & KPachonska & 3/17/20 2:15:00 PM \\
\hline \multicolumn{3}{|l|}{ Font: $12 \mathrm{pt}$} \\
\hline Page 9: [829] Formatted & KPachonska & 3/17/20 2:15:00 PM \\
\hline \multicolumn{3}{|l|}{ Font: $12 \mathrm{pt}$} \\
\hline Page 9: [830] Formatted & KPachonska & 3/17/20 2:15:00 PM \\
\hline \multicolumn{3}{|l|}{ Font: $12 \mathrm{pt}$} \\
\hline Page 9: [831] Formatted & KPachonska & 3/17/20 2:15:00 PM \\
\hline \multicolumn{3}{|l|}{ Font: $12 \mathrm{pt}$} \\
\hline Page 9: [831] Formatted & KPachonska & 3/17/20 2:15:00 PM \\
\hline \multicolumn{3}{|l|}{ Font: $12 \mathrm{pt}$} \\
\hline Page 9: [832] Formatted & KPachonska & 3/17/20 2:15:00 PM \\
\hline \multicolumn{3}{|l|}{ Font: $12 \mathrm{pt}$} \\
\hline Page 9: [833] Formatted & KPachonska & 3/17/20 2:15:00 PM \\
\hline \multicolumn{3}{|l|}{ Font: $12 \mathrm{pt}$} \\
\hline Page 9: [833] Formatted & KPachonska & 3/17/20 2:15:00 PM \\
\hline
\end{tabular}


Font: $12 \mathrm{pt}$

Page 9: [834] Formatted

KPachonska

3/17/20 2:15:00 PM

Font: $12 \mathrm{pt}$

Page 9: [835] Formatted

KPachonska

3/17/20 2:15:00 PM

Font: $12 \mathrm{pt}$

Page 9: [836] Formatted

KPachonska

3/17/20 2:15:00 PM

Font: $12 \mathrm{pt}$

Page 9: [837] Formatted

KPachonska

3/17/20 2:15:00 PM

Font: $12 \mathrm{pt}$

Page 9: [838] Formatted

KPachonska

3/17/20 2:15:00 PM

Font: $12 \mathrm{pt}$

Page 9: [839] Formatted

KPachonska

3/17/20 2:15:00 PM

Font: $12 \mathrm{pt}$

Page 9: [840] Formatted

KPachonska

3/17/20 2:15:00 PM

Font: $12 \mathrm{pt}$

Page 9: [841] Formatted

KPachonska

3/17/20 2:15:00 PM

Font: $12 \mathrm{pt}$

Page 9: [842] Formatted

KPachonska

3/17/20 2:15:00 PM

Font: $12 \mathrm{pt}$

Page 9: [843] Formatted

KPachonska

3/17/20 2:15:00 PM

Font: $12 \mathrm{pt}$

Page 9: [844] Formatted

KPachonska

3/17/20 2:15:00 PM

Font: $12 \mathrm{pt}$

Page 9: [845] Formatted

KPachonska

3/17/20 2:15:00 PM

Font: $12 \mathrm{pt}$

Page 9: [846] Formatted

KPachonska

3/17/20 2:15:00 PM

Font: $12 \mathrm{pt}$

Page 9: [847] Formatted

KPachonska

3/17/20 2:15:00 PM

Font: $12 \mathrm{pt}$

Page 9: [848] Formatted

KPachonska

3/17/20 2:15:00 PM

Font: $12 \mathrm{pt}$

Page 1: [849] Formatted

KPachonska

3/17/20 2:15:00 PM

Normal, Tab stops: 6.26", Right

Page 1: [850] Formatted

KPachonska

3/17/20 2:15:00 PM

Normal, Tab stops: 6.26", Right

Page 10: [851] Formatted

KPachonska

3/17/20 2:15:00 PM

Font: $12 \mathrm{pt}$

Page 10: [852] Deleted

KPachonska

3/17/20 2:15:00 PM 


\begin{tabular}{|c|c|c|}
\hline Page 10: [853] Formatted & KPachonska & 3/17/20 2:15:00 PM \\
\hline \multicolumn{3}{|l|}{ Font: $12 \mathrm{pt}$} \\
\hline Page 10: [854] Deleted & KPachonska & 3/17/20 2:15:00 PM \\
\hline \multicolumn{3}{|l|}{$\nabla \ldots$} \\
\hline Page 10: [855] Formatted & KPachonska & 3/17/20 2:15:00 PM \\
\hline \multicolumn{3}{|l|}{ Font: $12 \mathrm{pt}$} \\
\hline Page 10: [856] Formatted & KPachonska & 3/17/20 2:15:00 PM \\
\hline \multicolumn{3}{|l|}{ Font: $12 \mathrm{pt}$} \\
\hline Page 10: [857] Formatted & KPachonska & 3/17/20 2:15:00 PM \\
\hline \multicolumn{3}{|l|}{ Font: $12 \mathrm{pt}$} \\
\hline Page 10: $[858]$ Formatted & KPachonska & 3/17/20 2:15:00 PM \\
\hline \multicolumn{3}{|l|}{ Font: $12 \mathrm{pt}$} \\
\hline Page 10: $[858]$ Formatted & KPachonska & 3/17/20 2:15:00 PM \\
\hline \multicolumn{3}{|l|}{ Font: $12 \mathrm{pt}$} \\
\hline Page 10: [859] Formatted & KPachonska & 3/17/20 2:15:00 PM \\
\hline \multicolumn{3}{|l|}{ Font: $12 \mathrm{pt}$} \\
\hline Page 10: $[860]$ Formatted & KPachonska & 3/17/20 2:15:00 PM \\
\hline \multicolumn{3}{|l|}{ Font: $12 \mathrm{pt}$} \\
\hline Page 10: [861] Formatted & KPachonska & 3/17/20 2:15:00 PM \\
\hline \multicolumn{3}{|l|}{ Font: $12 \mathrm{pt}$} \\
\hline Page 10: [862] Formatted & KPachonska & 3/17/20 2:15:00 PM \\
\hline \multicolumn{3}{|l|}{ Font: $12 \mathrm{pt}$} \\
\hline Page 10: [863] Formatted & KPachonska & 3/17/20 2:15:00 PM \\
\hline \multicolumn{3}{|l|}{ Font: $12 \mathrm{pt}$} \\
\hline Page 10: [864] Formatted & KPachonska & 3/17/20 2:15:00 PM \\
\hline \multicolumn{3}{|l|}{ Font: $12 \mathrm{pt}$} \\
\hline Page 10: [865] Deleted & KPachonska & 3/17/20 2:15:00 PM \\
\hline \multicolumn{3}{|l|}{$\nabla$} \\
\hline Page 10: [866] Formatted & KPachonska & 3/17/20 2:15:00 PM \\
\hline \multicolumn{3}{|l|}{ Font: $12 \mathrm{pt}$} \\
\hline Page 10: [867] Formatted & KPachonska & 3/17/20 2:15:00 PM \\
\hline \multicolumn{3}{|l|}{ Font: $12 \mathrm{pt}$} \\
\hline Page 10: [868] Formatted & KPachonska & 3/17/20 2:15:00 PM \\
\hline \multicolumn{3}{|l|}{ Font: $12 \mathrm{pt}$} \\
\hline Page 10: [869] Formatted & KPachonska & 3/17/20 2:15:00 PM \\
\hline \multicolumn{3}{|l|}{ Font: $12 \mathrm{pt}$} \\
\hline Page 10: [870] Formatted & KPachonska & 3/17/20 2:15:00 PM \\
\hline \multicolumn{3}{|l|}{ Font: $12 \mathrm{pt}$} \\
\hline Page 10: [871] Formatted & KPachonska & 3/17/20 2:15:00 PM \\
\hline
\end{tabular}


Font: $12 \mathrm{pt}$

Page 10: [872] Formatted

KPachonska

3/17/20 2:15:00 PM

Font: $12 \mathrm{pt}$

Page 10: [873] Formatted

KPachonska

3/17/20 2:15:00 PM

Font: $12 \mathrm{pt}$

Page 10: [873] Formatted

KPachonska

3/17/20 2:15:00 PM

Font: $12 \mathrm{pt}$

Page 10: [874] Formatte

KPachonska

3/17/20 2:15:00 PM

Font: $12 \mathrm{pt}$

Page 10: [875] Formatted

KPachonska

3/17/20 2:15:00 PM

Font: $12 \mathrm{pt}$

Page 10: [876] Formatted

KPachonska

3/17/20 2:15:00 PM

Font: $12 \mathrm{pt}$

Page 10: [877] Formatted

KPachonska

3/17/20 2:15:00 PM

Font: $12 \mathrm{pt}$

Page 10: [878] Formatted

KPachonska

3/17/20 2:15:00 PM

Font: $12 \mathrm{pt}$

Page 10: [879] Formatted

KPachonska

3/17/20 2:15:00 PM

Font: $12 \mathrm{pt}$

Page 10: [880] Formatted

KPachonska

3/17/20 2:15:00 PM

Font: $12 \mathrm{pt}$

Page 10: [881] Commented [JMS6] Julia Massimelli Sewall

3/24/20 9:26:00 PM

rewrite summarizing your findings better. "Usefulness" is not descriptive

\begin{tabular}{|c|c|c|}
\hline Page 10: [882] Formatted & KPachonska & 3/17/20 2:15:00 PM \\
\hline \multicolumn{3}{|l|}{ Font: $12 \mathrm{pt}$} \\
\hline Page 10: [883] Formatted & KPachonska & 3/17/20 2:15:00 PM \\
\hline \multicolumn{3}{|l|}{ Font: $12 \mathrm{pt}$} \\
\hline Page 10: [884] Formatted & KPachonska & 3/17/20 2:15:00 PM \\
\hline \multicolumn{3}{|l|}{ Font: $12 \mathrm{pt}$} \\
\hline Page 10: [885] Formatted & KPachonska & 3/17/20 2:15:00 PM \\
\hline \multicolumn{3}{|l|}{ Font: $12 \mathrm{pt}$} \\
\hline Page 10: [886] Formatted & KPachonska & 3/17/20 2:15:00 PM \\
\hline \multicolumn{3}{|l|}{ Font: $12 \mathrm{pt}$} \\
\hline Page 10: [887] Formatted & KPachonska & 3/17/20 2:15:00 PM \\
\hline \multicolumn{3}{|l|}{ Font: $12 \mathrm{pt}$} \\
\hline Page 10: [888] Formatted & KPachonska & 3/17/20 2:15:00 PM \\
\hline \multicolumn{3}{|l|}{ Font: $12 \mathrm{pt}$} \\
\hline Page 10: [889] Formatted & KPachonska & 3/17/20 2:15:00 PM \\
\hline \multicolumn{3}{|l|}{ Font: $12 \mathrm{pt}$} \\
\hline Page 10: $[890]$ Formatted & KPachonska & 3/17/20 2:15:00 PM \\
\hline
\end{tabular}


Font: $12 \mathrm{pt}$

Page 10: [891] Formatted

KPachonska

3/17/20 2:15:00 PM

Font: $12 \mathrm{pt}$

Page 10: [892] Formatted

KPachonska

3/17/20 2:15:00 PM

Font: $12 \mathrm{pt}$

Page 10: [893] Deleted

KPachonska

3/17/20 2:15:00 PM

Page 10: [894] Formatted

KPachonska

3/17/20 2:15:00 PM

Font: $12 \mathrm{pt}$

Page 10: [895] Formatted

KPachonska

3/17/20 2:15:00 PM

Font: $12 \mathrm{pt}$

Page 10: [896] Formatted

KPachonska

3/17/20 2:15:00 PM

Font: $12 \mathrm{pt}$

Page 10: [897] Formatted

KPachonska

3/17/20 2:15:00 PM

Font: $12 \mathrm{pt}$

Page 10: [898] Formatted

KPachonska

3/17/20 2:15:00 PM

Font: $12 \mathrm{pt}$

Page 10: [899] Formatted

KPachonska

3/17/20 2:15:00 PM

Font: $12 \mathrm{pt}$

Page 10: [900] Formatted

KPachonska

3/17/20 2:15:00 PM

Font: $12 \mathrm{pt}$

Page 10: [901] Formatted

KPachonska

3/17/20 2:15:00 PM

Font: $12 \mathrm{pt}$

Page 10: [902] Formatted

KPachonska

3/17/20 2:15:00 PM

Font: $12 \mathrm{pt}$

Page 10: [903] Formatte

KPachonska

3/17/20 2:15:00 PM

Font: $12 \mathrm{pt}$

Page 10: [903] Formatted

Font: $12 \mathrm{pt}$

Page 10: [904] Formatte

KPachonska

3/17/20 2:15:00 PM

Font: $12 \mathrm{pt}$

Page 10: [905] Formatted

KPachonska

3/17/20 2:15:00 PM

Font: $12 \mathrm{pt}$

Page 10: [906] Formatted

KPachonska

3/17/20 2:15:00 PM

Font: $12 \mathrm{pt}$

Page 10: [907] Formatted

KPachonska

3/17/20 2:15:00 PM

Font: $12 \mathrm{pt}$

Page 10: [908] Formatted

KPachonska

3/17/20 2:15:00 PM

Font: $12 \mathrm{pt}$ 


\begin{tabular}{|c|c|c|}
\hline Page 10: [909] Formatted & KPachonska & 3/17/20 2:15:00 PM \\
\hline \multicolumn{3}{|l|}{ Font: $12 \mathrm{pt}$} \\
\hline Page 10: [910] Formatted & KPachonska & 3/17/20 2:15:00 PM \\
\hline \multicolumn{3}{|l|}{ Font: $12 \mathrm{pt}$} \\
\hline Page 10: [911] Formatted & KPachonska & 3/17/20 2:15:00 PM \\
\hline \multicolumn{3}{|l|}{ Font: $12 \mathrm{pt}$} \\
\hline Page 10: [911] Formatted & KPachonska & 3/17/20 2:15:00 PM \\
\hline \multicolumn{3}{|l|}{ Font: $12 \mathrm{pt}$} \\
\hline Page 10: [911] Formatted & KPachonska & 3/17/20 2:15:00 PM \\
\hline \multicolumn{3}{|l|}{ Font: $12 \mathrm{pt}$} \\
\hline Page 10: [912] Formatted & KPachonska & 3/17/20 2:15:00 PM \\
\hline \multicolumn{3}{|l|}{ Font: $12 \mathrm{pt}$} \\
\hline Page 10: [913] Deleted & KPachonska & 3/17/20 2:15:00 PM \\
\hline \multicolumn{3}{|l|}{$\nabla$} \\
\hline Page 10: [914] Formatted & KPachonska & 3/17/20 2:15:00 PM \\
\hline \multicolumn{3}{|l|}{ Font: $12 \mathrm{pt}$} \\
\hline Page 10: $[914]$ Formatted & KPachonska & 3/17/20 2:15:00 PM \\
\hline \multicolumn{3}{|l|}{ Font: $12 \mathrm{pt}$} \\
\hline Page 10: [914] Formatted & KPachonska & 3/17/20 2:15:00 PM \\
\hline \multicolumn{3}{|l|}{ Font: $12 \mathrm{pt}$} \\
\hline Page 10: $[915]$ Formatted & KPachonska & 3/17/20 2:15:00 PM \\
\hline \multicolumn{3}{|l|}{ Font: $12 \mathrm{pt}$} \\
\hline Page 10: [916] Formatted & KPachonska & 3/17/20 2:15:00 PM \\
\hline \multicolumn{3}{|l|}{ Font: $12 \mathrm{pt}$} \\
\hline Page 10: [916] Formatted & KPachonska & 3/17/20 2:15:00 PM \\
\hline \multicolumn{3}{|l|}{ Font: $12 \mathrm{pt}$} \\
\hline Page 10: [917] Deleted & KPachonska & 3/17/20 2:15:00 PM \\
\hline$\checkmark \ldots \ldots \ldots \ldots$ & & \\
\hline Page 10: $[918]$ Formatted & KPachonska & 3/17/20 2:15:00 PM \\
\hline \multicolumn{3}{|l|}{ Font: $12 \mathrm{pt}$} \\
\hline Page 10: [919] Formatted & KPachonska & 3/17/20 2:15:00 PM \\
\hline \multicolumn{3}{|l|}{ Font: $12 \mathrm{pt}$} \\
\hline Page 10: [920] Formatted & KPachonska & 3/17/20 2:15:00 PM \\
\hline \multicolumn{3}{|l|}{ Font: $12 \mathrm{pt}$} \\
\hline Page 10: [921] Formatted & KPachonska & 3/17/20 2:15:00 PM \\
\hline \multicolumn{3}{|l|}{ Font: $12 \mathrm{pt}$} \\
\hline Page 10: [922] Formatted & KPachonska & 3/17/20 2:15:00 PM \\
\hline \multicolumn{3}{|l|}{ Font: $12 \mathrm{pt}$} \\
\hline Page 10: [923] Formatted & KPachonska & 3/17/20 2:15:00 PM \\
\hline
\end{tabular}


Font: $12 \mathrm{pt}$

Page 10: [924] Formatted

KPachonska

3/17/20 2:15:00 PM

Font: $12 \mathrm{pt}$

Page 10: [925] Formatted

KPachonska

3/17/20 2:15:00 PM

Font: $12 \mathrm{pt}$

Page 10: [926] Formatted

KPachonska

3/17/20 2:15:00 PM

Font: $12 \mathrm{pt}$

Page 10: [927] Formatted

KPachonska

3/17/20 2:15:00 PM

Font: $12 \mathrm{pt}$

Page 10: [927] Formatte

KPachonska

3/17/20 2:15:00 PM

Font: $12 \mathrm{pt}$

Page 10: [928] Formatted

KPachonska

3/17/20 2:15:00 PM

Font: $12 \mathrm{pt}$

Page 10: [929] Formatted

KPachonska

3/17/20 2:15:00 PM

Font: $12 \mathrm{pt}$

Page 10: [930] Formatted

KPachonska

3/17/20 2:15:00 PM

Font: $12 \mathrm{pt}$

Page 10: [931] Formatted

KPachonska

3/17/20 2:15:00 PM

Font: $12 \mathrm{pt}$

Page 10: [932] Formatted

KPachonska

3/17/20 2:15:00 PM

Font: $12 \mathrm{pt}$

Page 10: [933] Formatte

KPachonska

3/17/20 2:15:00 PM

Font: $12 \mathrm{pt}$

Page 10: [934] Formatted

Font: $12 \mathrm{pt}$

Page 10: [934] Formatted

KPachonska

3/17/20 2:15:00 PM

Font: $12 \mathrm{pt}$

Page 10: [935] Formatted

KPachonska

3/17/20 2:15:00 PM

Font: $12 \mathrm{pt}$

Page 10: [936] Formatted

KPachonska

3/17/20 2:15:00 PM

Font: $12 \mathrm{pt}$

Page 10: [937] Formatted

KPachonska

3/17/20 2:15:00 PM

Font: $12 \mathrm{pt}$

Page 10: [938] Formatted

KPachonska

3/17/20 2:15:00 PM

Font: $12 \mathrm{pt}$

Page 10: [939] Formatte

KPachonska

3/17/20 2:15:00 PM

Font: $12 \mathrm{pt}$

Page 10: [940] Formatted

KPachonska

3/17/20 2:15:00 PM

Font: $12 \mathrm{pt}$

Page 10: [941] Deleted

KPachonska

3/17/20 2:15:00 PM 


\begin{tabular}{|c|c|c|}
\hline Page 10: $[942]$ Formatted & KPachonska & 3/17/20 2:15:00 PM \\
\hline \multicolumn{3}{|l|}{ Font: $12 \mathrm{pt}$} \\
\hline Page 10: [943] Formatted & KPachonska & 3/17/20 2:15:00 PM \\
\hline \multicolumn{3}{|l|}{ Font: $12 \mathrm{pt}$} \\
\hline Page 10: [943] Formatted & KPachonska & 3/17/20 2:15:00 PM \\
\hline \multicolumn{3}{|l|}{ Font: $12 \mathrm{pt}$} \\
\hline Page 10: [944] Deleted & KPachonska & 3/17/20 2:15:00 PM \\
\hline \multicolumn{3}{|l|}{ 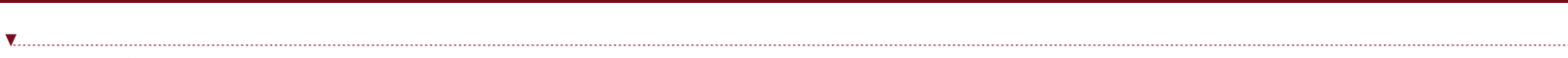 } \\
\hline Page 10: [945] Formatted & KPachonska & 3/17/20 2:15:00 PM \\
\hline \multicolumn{3}{|l|}{ Font: $12 \mathrm{pt}$} \\
\hline Page 10: [946] Formatted & KPachonska & 3/17/20 2:15:00 PM \\
\hline \multicolumn{3}{|l|}{ Font: $12 \mathrm{pt}$} \\
\hline Page 10: [947] Formatted & KPachonska & 3/17/20 2:15:00 PM \\
\hline \multicolumn{3}{|l|}{ Font: $12 \mathrm{pt}$} \\
\hline Page 10: $[948]$ Formatted & KPachonska & 3/17/20 2:15:00 PM \\
\hline \multicolumn{3}{|l|}{ Font: $12 \mathrm{pt}$} \\
\hline Page 10: [949] Formatted & KPachonska & 3/17/20 2:15:00 PM \\
\hline \multicolumn{3}{|l|}{ Font: $12 \mathrm{pt}$} \\
\hline Page 10: $[950]$ Formatted & KPachonska & 3/17/20 2:15:00 PM \\
\hline \multicolumn{3}{|l|}{ Font: $12 \mathrm{pt}$} \\
\hline Page 10: [951] Formatted & KPachonska & 3/17/20 2:15:00 PM \\
\hline \multicolumn{3}{|l|}{ Font: $12 \mathrm{pt}$} \\
\hline Page 10: [952] Formatted & KPachonska & 3/17/20 2:15:00 PM \\
\hline \multicolumn{3}{|l|}{ Font: $12 \mathrm{pt}$} \\
\hline Page 10: [953] Formatted & KPachonska & 3/17/20 2:15:00 PM \\
\hline \multicolumn{3}{|l|}{ Font: $12 \mathrm{pt}$} \\
\hline Page 10: [954] Formatted & KPachonska & 3/17/20 2:15:00 PM \\
\hline \multicolumn{3}{|l|}{ Font: $12 \mathrm{pt}$} \\
\hline Page 10: [955] Formatted & KPachonska & 3/17/20 2:15:00 PM \\
\hline \multicolumn{3}{|l|}{ Font: $12 \mathrm{pt}$} \\
\hline Page 10: [956] Formatted & KPachonska & 3/17/20 2:15:00 PM \\
\hline \multicolumn{3}{|l|}{ Font: $12 \mathrm{pt}$} \\
\hline Page 10: [957] Formatted & KPachonska & 3/17/20 2:15:00 PM \\
\hline \multicolumn{3}{|l|}{ Font: $12 \mathrm{pt}$} \\
\hline Page 10: [958] Formatted & KPachonska & 3/17/20 2:15:00 PM \\
\hline \multicolumn{3}{|l|}{ Font: $12 \mathrm{pt}$} \\
\hline Page 10: [959] Formatted & KPachonska & 3/17/20 2:15:00 PM \\
\hline
\end{tabular}




\begin{tabular}{|c|c|c|}
\hline Page 10: [960] Formatted & KPachonska & 3/17/20 2:15:00 PM \\
\hline \multicolumn{3}{|l|}{ Font: $12 \mathrm{pt}$} \\
\hline Page 10: [961] Formatted & KPachonska & 3/17/20 2:15:00 PM \\
\hline \multicolumn{3}{|l|}{ Font: $12 \mathrm{pt}$} \\
\hline Page 10: $[962]$ Formatted & KPachonska & 3/17/20 2:15:00 PM \\
\hline \multicolumn{3}{|l|}{ Font: $12 \mathrm{pt}$} \\
\hline Page 10: [963] Formatted & KPachonska & 3/17/20 2:15:00 PM \\
\hline \multicolumn{3}{|l|}{ Font: $12 \mathrm{pt}$} \\
\hline Page 10: $[964]$ Formatted & KPachonska & 3/17/20 2:15:00 PM \\
\hline \multicolumn{3}{|l|}{ Font: $12 \mathrm{pt}$} \\
\hline Page 10: $[965]$ Formatted & KPachonska & 3/17/20 2:15:00 PM \\
\hline \multicolumn{3}{|l|}{ Font: $12 \mathrm{pt}$} \\
\hline Page 10: [966] Formatted & KPachonska & 3/17/20 2:15:00 PM \\
\hline \multicolumn{3}{|l|}{ Font: $12 \mathrm{pt}$} \\
\hline Page 10: [967] Formatted & KPachonska & 3/17/20 2:15:00 PM \\
\hline \multicolumn{3}{|l|}{ Font: $12 \mathrm{pt}$} \\
\hline Page 10: [968] Formatted & KPachonska & 3/17/20 2:15:00 PM \\
\hline \multicolumn{3}{|l|}{ Font: $12 \mathrm{pt}$} \\
\hline Page 10: $[969]$ Formatted & KPachonska & 3/17/20 2:15:00 PM \\
\hline \multicolumn{3}{|l|}{ Font: $12 \mathrm{pt}$} \\
\hline Page 10: [969] Formatted & KPachonska & 3/17/20 2:15:00 PM \\
\hline \multicolumn{3}{|l|}{ Font: $12 \mathrm{pt}$} \\
\hline Page 10: [970] Formatted & KPachonska & 3/17/20 2:15:00 PM \\
\hline \multicolumn{3}{|l|}{ Font: $12 \mathrm{pt}$} \\
\hline Page 10: $[971]$ Formatted & KPachonska & 3/17/20 2:15:00 PM \\
\hline \multicolumn{3}{|l|}{ Font: $12 \mathrm{pt}$} \\
\hline Page 10: [972] Formatted & KPachonska & 3/17/20 2:15:00 PM \\
\hline \multicolumn{3}{|l|}{ Font: $12 \mathrm{pt}$} \\
\hline Page 10: [973] Formatted & KPachonska & 3/17/20 2:15:00 PM \\
\hline \multicolumn{3}{|l|}{ Font: $12 \mathrm{pt}$} \\
\hline Page 10: [974] Formatted & KPachonska & 3/17/20 2:15:00 PM \\
\hline \multicolumn{3}{|l|}{ Font: $12 \mathrm{pt}$} \\
\hline Page 10: $[975]$ Formatted & KPachonska & 3/17/20 2:15:00 PM \\
\hline \multicolumn{3}{|l|}{ Font: $12 \mathrm{pt}$} \\
\hline Page 10: [976] Formatted & KPachonska & 3/17/20 2:15:00 PM \\
\hline \multicolumn{3}{|l|}{ Font: $12 \mathrm{pt}$} \\
\hline Page 10: [977] Formatted & KPachonska & 3/17/20 2:15:00 PM \\
\hline \multicolumn{3}{|l|}{ Font: $12 \mathrm{pt}$} \\
\hline Page 10: $[978]$ Formatted & KPachonska & 3/17/20 2:15:00 PM \\
\hline
\end{tabular}




\begin{tabular}{|c|c|c|}
\hline Page 10: [979] Formatted & KPachonska & 3/17/20 2:15:00 PM \\
\hline \multicolumn{3}{|l|}{ Font: $12 \mathrm{pt}$} \\
\hline Page 10: [980] Deleted & KPachonska & 3/17/20 2:15:00 PM \\
\hline \multicolumn{3}{|l|}{$\nabla$} \\
\hline Page 10: [981] Formatted & KPachonska & 3/17/20 2:15:00 PM \\
\hline \multicolumn{3}{|l|}{ Font: $12 \mathrm{pt}$} \\
\hline Page 10: [982] Formatted & KPachonska & 3/17/20 2:15:00 PM \\
\hline \multicolumn{3}{|l|}{ Font: $12 \mathrm{pt}$} \\
\hline Page 10: [983] Formatted & KPachonska & 3/17/20 2:15:00 PM \\
\hline \multicolumn{3}{|l|}{ Font: $12 \mathrm{pt}$} \\
\hline Page 10: $[984]$ Formatted & KPachonska & 3/17/20 2:15:00 PM \\
\hline \multicolumn{3}{|l|}{ Font: $12 \mathrm{pt}$} \\
\hline Page 10: [985] Formatted & KPachonska & 3/17/20 2:15:00 PM \\
\hline \multicolumn{3}{|l|}{ Font: $12 \mathrm{pt}$} \\
\hline Page 10: [986] Formatted & KPachonska & 3/17/20 2:15:00 PM \\
\hline \multicolumn{3}{|l|}{ Font: $12 \mathrm{pt}$} \\
\hline Page 10: [987] Formatted & KPachonska & 3/17/20 2:15:00 PM \\
\hline \multicolumn{3}{|l|}{ Font: $12 \mathrm{pt}$} \\
\hline Page 1: $[988]$ Formatted & KPachonska & 3/17/20 2:15:00 PM \\
\hline \multicolumn{3}{|c|}{ Normal, Tab stops: $6.26 "$, Right } \\
\hline Page 1: [989] Formatted & KPachonska & 3/17/20 2:15:00 PM \\
\hline \multicolumn{3}{|c|}{ Normal, Tab stops: 6.26", Right } \\
\hline Page 11: [990] Deleted & KPachonska & 3/17/20 2:15:00 PM \\
\hline Page 11: [991] Formatted & KPachonska & 3/17/20 2:15:00 PM \\
\hline \multicolumn{3}{|l|}{ Font: $12 \mathrm{pt}$} \\
\hline Page 11: [992] Formatted & KPachonska & 3/17/20 2:15:00 PM \\
\hline \multicolumn{3}{|l|}{ Font: $12 \mathrm{pt}$} \\
\hline Page 11: [993] Formatted & KPachonska & 3/17/20 2:15:00 PM \\
\hline \multicolumn{3}{|l|}{ Font: $12 \mathrm{pt}$} \\
\hline Page 11: [993] Formatted & KPachonska & 3/17/20 2:15:00 PM \\
\hline \multicolumn{3}{|l|}{ Font: $12 \mathrm{pt}$} \\
\hline Page 11: [994] Formatted & KPachonska & 3/17/20 2:15:00 PM \\
\hline \multicolumn{3}{|l|}{ Font: $12 \mathrm{pt}$} \\
\hline Page 11: [994] Formatted & KPachonska & 3/17/20 2:15:00 PM \\
\hline \multicolumn{3}{|l|}{ Font: $12 \mathrm{pt}$} \\
\hline Page 11: [995] Formatted & KPachonska & 3/17/20 2:15:00 PM \\
\hline \multicolumn{3}{|l|}{ Font: $12 \mathrm{pt}$} \\
\hline Page 11: [996] Formatted & KPachonska & 3/17/20 2:15:00 PM \\
\hline
\end{tabular}

Font: $12 \mathrm{pt}$ 


\begin{tabular}{|c|c|c|}
\hline Page 11: [997] Formatted & KPachonska & 3/17/20 2:15:00 PM \\
\hline \multicolumn{3}{|l|}{ Font: $12 \mathrm{pt}$} \\
\hline Page 11: [998] Formatted & KPachonska & 3/17/20 2:15:00 PM \\
\hline \multicolumn{3}{|l|}{ Font: $12 \mathrm{pt}$} \\
\hline Page 11: [999] Formatted & KPachonska & 3/17/20 2:15:00 PM \\
\hline \multicolumn{3}{|l|}{ Font: $12 \mathrm{pt}$} \\
\hline Page 11: [1000] Formatted & KPachonska & 3/17/20 2:15:00 PM \\
\hline \multicolumn{3}{|l|}{ Font: $12 \mathrm{pt}$} \\
\hline Page 11: [1001] Formatted & KPachonska & 3/17/20 2:15:00 PM \\
\hline \multicolumn{3}{|l|}{ Font color: Black } \\
\hline Page 11: [1001] Formatted & KPachonska & 3/17/20 2:15:00 PM \\
\hline \multicolumn{3}{|l|}{ Font color: Black } \\
\hline Page 11: [1002] Formatted & KPachonska & 3/17/20 2:15:00 PM \\
\hline \multicolumn{3}{|c|}{ Font: 12 pt, Font color: Black, English (US) } \\
\hline Page 11: [1003] Formatted & KPachonska & 3/17/20 2:15:00 PM \\
\hline \multicolumn{3}{|c|}{ Font: 12 pt, Font color: Black, English (US) } \\
\hline Page 11: [1003] Formatted & KPachonska & 3/17/20 2:15:00 PM \\
\hline \multicolumn{3}{|c|}{ Font: 12 pt, Font color: Black, English (US) } \\
\hline Page 11: $[1004]$ Formatted & KPachonska & 3/17/20 2:15:00 PM \\
\hline \multicolumn{3}{|l|}{ Font: 12 pt, English (US) } \\
\hline Page 11: [1004] Formatted & KPachonska & 3/17/20 2:15:00 PM \\
\hline \multicolumn{3}{|l|}{ Font: 12 pt, English (US) } \\
\hline Page 11: [1004] Formatted & KPachonska & 3/17/20 2:15:00 PM \\
\hline \multicolumn{3}{|l|}{ Font: 12 pt, English (US) } \\
\hline Page 11: [1005] Change & Unknown & \\
\hline \multicolumn{3}{|l|}{ Field Code Changed } \\
\hline Page 11: [1006] Formatted & KPachonska & 3/17/20 2:15:00 PM \\
\hline \multicolumn{3}{|c|}{ Font: 12 pt, Font color: Black } \\
\hline Page 11: [1007] Formatted & KPachonska & 3/17/20 2:15:00 PM \\
\hline \multicolumn{3}{|c|}{ Font: 12 pt, Font color: Black } \\
\hline Page 11: [1008] Formatted & KPachonska & 3/17/20 2:15:00 PM \\
\hline \multicolumn{3}{|c|}{ Font: 12 pt, Font color: Black } \\
\hline Page 11: [1009] Formatted & KPachonska & 3/17/20 2:15:00 PM \\
\hline \multicolumn{3}{|c|}{ Font: 12 pt, Font color: Black } \\
\hline Page 11: [1010] Formatted & KPachonska & 3/17/20 2:15:00 PM \\
\hline \multicolumn{3}{|c|}{ Font: 12 pt, Font color: Black } \\
\hline Page 11: [1011] Formatted & KPachonska & 3/17/20 2:15:00 PM \\
\hline \multicolumn{3}{|c|}{ Font: 12 pt, Font color: Black } \\
\hline Page 11: [1012] Formatted & KPachonska & 3/17/20 2:15:00 PM \\
\hline
\end{tabular}

Font: 12 pt, Font color: Black 


\begin{tabular}{|c|c|c|}
\hline Page 11: [1013] Formatted & KPachonska & 3/17/20 2:15:00 PM \\
\hline \multicolumn{3}{|l|}{ Font: 12 pt, Font color: Black } \\
\hline Page 11: [1014] Formatted & KPachonska & 3/17/20 2:15:00 PM \\
\hline \multicolumn{3}{|l|}{ Font: 12 pt, Font color: Black } \\
\hline Page 11: [1015] Formatted & KPachonska & 3/17/20 2:15:00 PM \\
\hline \multicolumn{3}{|l|}{ Font: 12 pt, Font color: Black } \\
\hline Page 11: [1016] Formatted & KPachonska & 3/17/20 2:15:00 PM \\
\hline \multicolumn{3}{|l|}{ Font: 12 pt, Font color: Black } \\
\hline Page 11: [1017] Formatted & KPachonska & 3/17/20 2:15:00 PM \\
\hline \multicolumn{3}{|l|}{ Font: $12 \mathrm{pt}$, Font color: Black } \\
\hline Page 11: [1018] Formatted & KPachonska & 3/17/20 2:15:00 PM \\
\hline \multicolumn{3}{|l|}{ Font: $12 \mathrm{pt}$, Font color: Black } \\
\hline Page 11: [1019] Formatted & KPachonska & 3/17/20 2:15:00 PM \\
\hline \multicolumn{3}{|l|}{ Font: 12 pt, Font color: Black } \\
\hline Page 11: $[1020]$ Formatted & KPachonska & 3/17/20 2:15:00 PM \\
\hline \multicolumn{3}{|l|}{ Font: 12 pt, Font color: Black } \\
\hline Page 11: [1021] Formatted & KPachonska & 3/17/20 2:15:00 PM \\
\hline \multicolumn{3}{|l|}{ Font: 12 pt, Font color: Black } \\
\hline Page 11: [1022] Formatted & KPachonska & 3/17/20 2:15:00 PM \\
\hline \multicolumn{3}{|l|}{ Font: 12 pt, Font color: Black } \\
\hline Page 11: [1023] Formatted & KPachonska & 3/17/20 2:15:00 PM \\
\hline \multicolumn{3}{|l|}{ Font: 12 pt, Font color: Black } \\
\hline Page 11: [1024] Formatted & KPachonska & 3/17/20 2:15:00 PM \\
\hline \multicolumn{3}{|l|}{ Font: 12 pt, Font color: Black } \\
\hline Page 11: [1025] Formatted & KPachonska & 3/17/20 2:15:00 PM \\
\hline \multicolumn{3}{|l|}{ Font: 12 pt, Font color: Black } \\
\hline Page 11: [1026] Formatted & KPachonska & 3/17/20 2:15:00 PM \\
\hline \multicolumn{3}{|l|}{ Font: 12 pt, Font color: Black } \\
\hline Page 11: [1027] Formatted & KPachonska & 3/17/20 2:15:00 PM \\
\hline \multicolumn{3}{|l|}{ Font: 12 pt, Font color: Black } \\
\hline Page 11: [1028] Formatted & KPachonska & 3/17/20 2:15:00 PM \\
\hline \multicolumn{3}{|l|}{ Font: 12 pt, Font color: Black } \\
\hline Page 11: [1029] Formatted & KPachonska & 3/17/20 2:15:00 PM \\
\hline \multicolumn{3}{|l|}{ Font: 12 pt, Font color: Black } \\
\hline Page 11: [1030] Formatted & KPachonska & 3/17/20 2:15:00 PM \\
\hline \multicolumn{3}{|l|}{ Font: 12 pt, Font color: Black } \\
\hline Page 11: [1031] Formatted & KPachonska & 3/17/20 2:15:00 PM \\
\hline \multicolumn{3}{|l|}{ Font: 12 pt, Font color: Black } \\
\hline Page 11: [1032] Formatted & KPachonska & 3/17/20 2:15:00 PM \\
\hline
\end{tabular}

Font: 12 pt, Font color: Black 


\begin{tabular}{|c|c|c|}
\hline Page 11: [1033] Formatted & KPachonska & 3/17/20 2:15:00 PM \\
\hline \multicolumn{3}{|l|}{ Font: 12 pt, Font color: Black } \\
\hline Page 11: [1034] Formatted & KPachonska & 3/17/20 2:15:00 PM \\
\hline \multicolumn{3}{|l|}{ Font: 12 pt, Font color: Black } \\
\hline Page 11: [1035] Formatted & KPachonska & 3/17/20 2:15:00 PM \\
\hline \multicolumn{3}{|l|}{ Font: 12 pt, Font color: Black } \\
\hline Page 11: [1036] Formatted & KPachonska & 3/17/20 2:15:00 PM \\
\hline \multicolumn{3}{|l|}{ Font: 12 pt, Font color: Black } \\
\hline Page 11: [1037] Formatted & KPachonska & 3/17/20 2:15:00 PM \\
\hline \multicolumn{3}{|l|}{ Font: $12 \mathrm{pt}$, Font color: Black } \\
\hline Page 11: [1038] Formatted & KPachonska & 3/17/20 2:15:00 PM \\
\hline \multicolumn{3}{|l|}{ Font: $12 \mathrm{pt}$, Font color: Black } \\
\hline Page 11: [1039] Formatted & KPachonska & 3/17/20 2:15:00 PM \\
\hline \multicolumn{3}{|l|}{ Font: 12 pt, Font color: Black } \\
\hline Page 11: [1040] Formatted & KPachonska & 3/17/20 2:15:00 PM \\
\hline \multicolumn{3}{|l|}{ Font: 12 pt, Font color: Black } \\
\hline Page 11: [1041] Formatted & KPachonska & 3/17/20 2:15:00 PM \\
\hline \multicolumn{3}{|l|}{ Font: 12 pt, Font color: Black } \\
\hline Page 11: $[1042]$ Formatted & KPachonska & 3/17/20 2:15:00 PM \\
\hline \multicolumn{3}{|l|}{ Font: 12 pt, Font color: Black } \\
\hline Page 11: [1043] Formatted & KPachonska & 3/17/20 2:15:00 PM \\
\hline \multicolumn{3}{|l|}{ Font: 12 pt, Font color: Black } \\
\hline Page 11: [1044] Formatted & KPachonska & 3/17/20 2:15:00 PM \\
\hline \multicolumn{3}{|l|}{ Font: 12 pt, Font color: Black } \\
\hline Page 11: [1045] Formatted & KPachonska & 3/17/20 2:15:00 PM \\
\hline \multicolumn{3}{|l|}{ Font: 12 pt, Font color: Black } \\
\hline Page 11: [1046] Formatted & KPachonska & 3/17/20 2:15:00 PM \\
\hline \multicolumn{3}{|l|}{ Font: 12 pt, Font color: Black } \\
\hline Page 11: [1047] Formatted & KPachonska & 3/17/20 2:15:00 PM \\
\hline \multicolumn{3}{|l|}{ Font: 12 pt, Font color: Black } \\
\hline Page 11: [1048] Formatted & KPachonska & 3/17/20 2:15:00 PM \\
\hline \multicolumn{3}{|l|}{ Font: 12 pt, Font color: Black } \\
\hline Page 11: [1049] Formatted & KPachonska & 3/17/20 2:15:00 PM \\
\hline \multicolumn{3}{|l|}{ Font: 12 pt, Font color: Black } \\
\hline Page 11: [1050] Formatted & KPachonska & 3/17/20 2:15:00 PM \\
\hline \multicolumn{3}{|l|}{ Font: 12 pt, Font color: Black } \\
\hline Page 11: [1051] Formatted & KPachonska & 3/17/20 2:15:00 PM \\
\hline \multicolumn{3}{|l|}{ Font: 12 pt, Font color: Black } \\
\hline Page 11: [1052] Formatted & KPachonska & 3/17/20 2:15:00 PM \\
\hline
\end{tabular}

Font: 12 pt, Font color: Black 


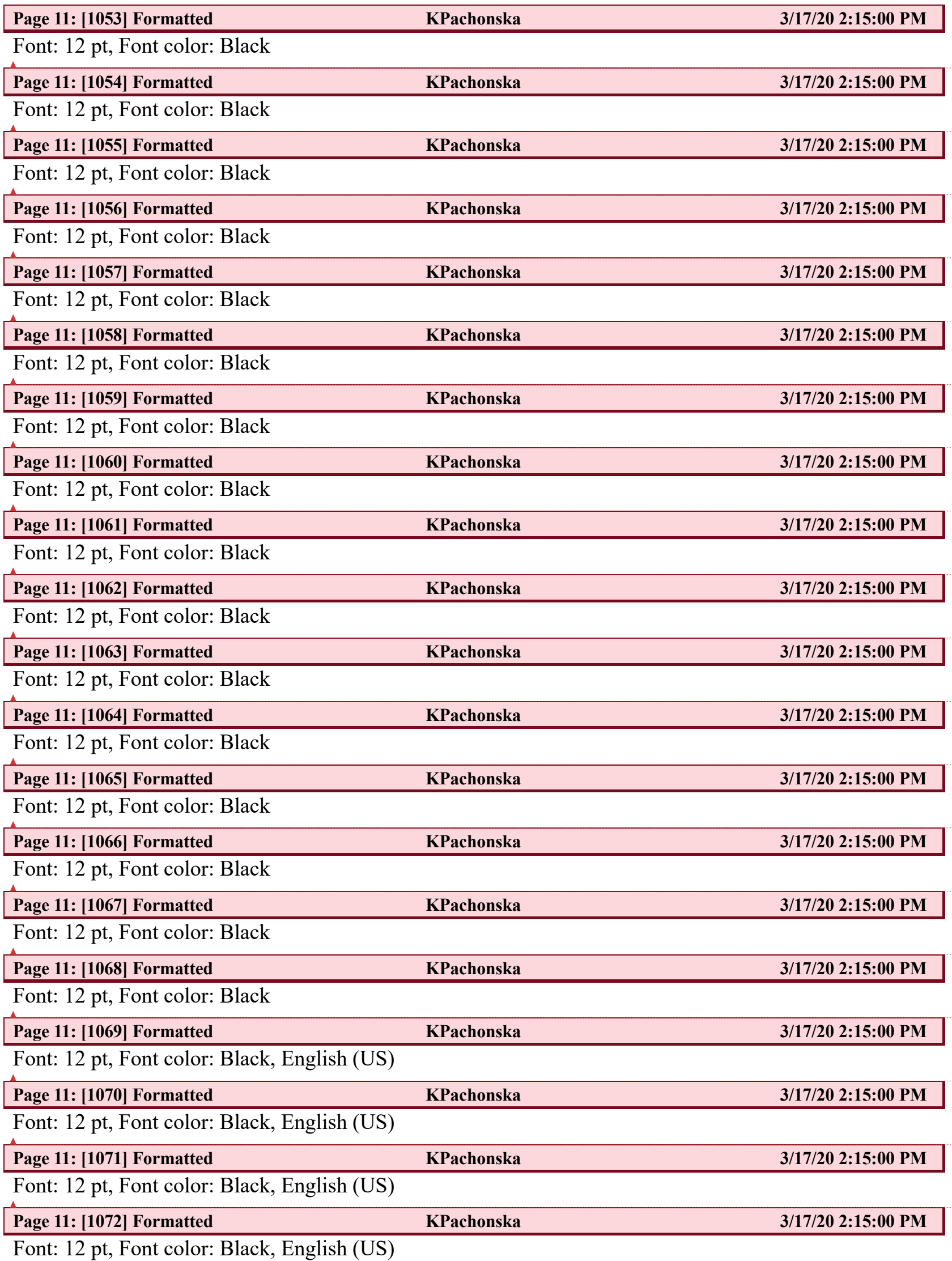




\begin{tabular}{|c|c|c|}
\hline Page 11: [1073] Formatted & KPachonska & 3/17/20 2:15:00 PM \\
\hline \multicolumn{3}{|l|}{ Font: 12 pt, Font color: Black } \\
\hline Page 11: [1074] Formatted & KPachonska & 3/17/20 2:15:00 PM \\
\hline \multicolumn{3}{|l|}{ Font: 12 pt, Font color: Black } \\
\hline Page 11: [1075] Formatted & KPachonska & 3/17/20 2:15:00 PM \\
\hline \multicolumn{3}{|l|}{ Font: 12 pt, Font color: Black } \\
\hline Page 11: [1076] Formatted & KPachonska & 3/17/20 2:15:00 PM \\
\hline \multicolumn{3}{|l|}{ Font: 12 pt, Font color: Black } \\
\hline Page 11: [1077] Formatted & KPachonska & 3/17/20 2:15:00 PM \\
\hline \multicolumn{3}{|l|}{ Font: $12 \mathrm{pt}$, Font color: Black } \\
\hline Page 11: [1078] Formatted & KPachonska & 3/17/20 2:15:00 PM \\
\hline \multicolumn{3}{|l|}{ Font: $12 \mathrm{pt}$, Font color: Black } \\
\hline Page 11: [1079] Formatted & KPachonska & 3/17/20 2:15:00 PM \\
\hline \multicolumn{3}{|l|}{ Font: 12 pt, Font color: Black } \\
\hline Page 11: [1080] Formatted & KPachonska & 3/17/20 2:15:00 PM \\
\hline \multicolumn{3}{|l|}{ Font: 12 pt, Font color: Black } \\
\hline Page 11: [1081] Formatted & KPachonska & 3/17/20 2:15:00 PM \\
\hline \multicolumn{3}{|l|}{ Font: 12 pt, Font color: Black } \\
\hline Page 11: [1082] Formatted & KPachonska & 3/17/20 2:15:00 PM \\
\hline \multicolumn{3}{|l|}{ Font: 12 pt, Font color: Black } \\
\hline Page 11: [1083] Formatted & KPachonska & 3/17/20 2:15:00 PM \\
\hline \multicolumn{3}{|l|}{ Font: 12 pt, Font color: Black } \\
\hline Page 11: [1084] Formatted & KPachonska & 3/17/20 2:15:00 PM \\
\hline \multicolumn{3}{|l|}{ Font: 12 pt, Font color: Black } \\
\hline Page 11: [1085] Formatted & KPachonska & 3/17/20 2:15:00 PM \\
\hline \multicolumn{3}{|l|}{ Font: 12 pt, Font color: Black } \\
\hline Page 11: [1086] Formatted & KPachonska & 3/17/20 2:15:00 PM \\
\hline \multicolumn{3}{|l|}{ Font: 12 pt, Font color: Black } \\
\hline Page 11: [1087] Formatted & KPachonska & 3/17/20 2:15:00 PM \\
\hline \multicolumn{3}{|l|}{ Font: 12 pt, Font color: Black } \\
\hline Page 11: [1088] Formatted & KPachonska & 3/17/20 2:15:00 PM \\
\hline \multicolumn{3}{|l|}{ Font: 12 pt, Font color: Black } \\
\hline Page 11: [1089] Formatted & KPachonska & 3/17/20 2:15:00 PM \\
\hline \multicolumn{3}{|l|}{ Font: 12 pt, Font color: Black } \\
\hline Page 11: [1090] Formatted & KPachonska & 3/17/20 2:15:00 PM \\
\hline \multicolumn{3}{|l|}{ Font: 12 pt, Font color: Black } \\
\hline Page 11: [1091] Formatted & KPachonska & 3/17/20 2:15:00 PM \\
\hline \multicolumn{3}{|l|}{ Font: 12 pt, Font color: Black } \\
\hline Page 11: [1092] Formatted & KPachonska & 3/17/20 2:15:00 PM \\
\hline
\end{tabular}

Font: 12 pt, Font color: Black 


\begin{tabular}{|c|c|c|}
\hline Page 11: [1093] Formatted & KPachonska & 3/17/20 2:15:00 PM \\
\hline \multicolumn{3}{|l|}{ Font: 12 pt, Font color: Black } \\
\hline Page 11: [1094] Formatted & KPachonska & 3/17/20 2:15:00 PM \\
\hline \multicolumn{3}{|l|}{ Font: 12 pt, Font color: Black } \\
\hline Page 11: [1095] Formatted & KPachonska & 3/17/20 2:15:00 PM \\
\hline \multicolumn{3}{|l|}{ Font: 12 pt, Font color: Black } \\
\hline Page 11: [1096] Formatted & KPachonska & 3/17/20 2:15:00 PM \\
\hline \multicolumn{3}{|l|}{ Font: $12 \mathrm{pt}$, Font color: Black } \\
\hline Page 11: [1097] Formatted & KPachonska & 3/17/20 2:15:00 PM \\
\hline \multicolumn{3}{|l|}{ Font: 12 pt, Font color: Black } \\
\hline Page 11: [1098] Formatted & KPachonska & 3/17/20 2:15:00 PM \\
\hline \multicolumn{3}{|l|}{ Font: 12 pt, Font color: Black } \\
\hline Page 11: [1099] Formatted & KPachonska & 3/17/20 2:15:00 PM \\
\hline \multicolumn{3}{|l|}{ Font: 12 pt, Font color: Black } \\
\hline Page 11: [1100] Formatted & KPachonska & 3/17/20 2:15:00 PM \\
\hline \multicolumn{3}{|l|}{ Font: 12 pt, Font color: Black } \\
\hline Page 11: [1101] Formatted & KPachonska & 3/17/20 2:15:00 PM \\
\hline \multicolumn{3}{|l|}{ Font: 12 pt, Font color: Black } \\
\hline Page 11: $[1102]$ Formatted & KPachonska & 3/17/20 2:15:00 PM \\
\hline \multicolumn{3}{|l|}{ Font: 12 pt, Font color: Black } \\
\hline Page 11: [1103] Formatted & KPachonska & 3/17/20 2:15:00 PM \\
\hline \multicolumn{3}{|l|}{ Font: 12 pt, Font color: Black } \\
\hline Page 11: [1104] Formatted & KPachonska & 3/17/20 2:15:00 PM \\
\hline \multicolumn{3}{|l|}{ Font: 12 pt, Font color: Black } \\
\hline Page 11: [1105] Formatted & KPachonska & 3/17/20 2:15:00 PM \\
\hline \multicolumn{3}{|l|}{ Font: 12 pt, Font color: Black } \\
\hline Page 11: [1106] Formatted & KPachonska & 3/17/20 2:15:00 PM \\
\hline \multicolumn{3}{|l|}{ Font: 12 pt, Font color: Black } \\
\hline Page 11: [1107] Formatted & KPachonska & 3/17/20 2:15:00 PM \\
\hline \multicolumn{3}{|l|}{ Font: 12 pt, Font color: Black } \\
\hline Page 11: [1108] Formatted & KPachonska & 3/17/20 2:15:00 PM \\
\hline \multicolumn{3}{|l|}{ Font: 12 pt, Font color: Black } \\
\hline Page 11: [1109] Formatted & KPachonska & 3/17/20 2:15:00 PM \\
\hline \multicolumn{3}{|l|}{ Font: 12 pt, Font color: Black } \\
\hline Page 11: [1110] Formatted & KPachonska & 3/17/20 2:15:00 PM \\
\hline \multicolumn{3}{|l|}{ Font: 12 pt, Font color: Black } \\
\hline Page 11: [1111] Formatted & KPachonska & 3/17/20 2:15:00 PM \\
\hline \multicolumn{3}{|l|}{ Font: 12 pt, Font color: Black } \\
\hline Page 11: [1112] Formatted & KPachonska & 3/17/20 2:15:00 PM \\
\hline
\end{tabular}

Font: 12 pt, Font color: Black 


\begin{tabular}{|c|c|c|}
\hline Page 11: [1113] Formatted & KPachonska & 3/17/20 2:15:00 PM \\
\hline \multicolumn{3}{|c|}{ Font: 12 pt, Font color: Black } \\
\hline Page 11: [1114] Formatted & KPachonska & 3/17/20 2:15:00 PM \\
\hline \multicolumn{3}{|c|}{ Font: 12 pt, Font color: Black } \\
\hline Page 11: [1115] Formatted & KPachonska & 3/17/20 2:15:00 PM \\
\hline \multicolumn{3}{|c|}{ Font: 12 pt, Font color: Black } \\
\hline Page 11: [1116] Formatted & KPachonska & 3/17/20 2:15:00 PM \\
\hline \multicolumn{3}{|c|}{ Font: 12 pt, Font color: Black } \\
\hline Page 11: [1117] Formatted & KPachonska & 3/17/20 2:15:00 PM \\
\hline \multicolumn{3}{|c|}{ Font: 12 pt, Font color: Black } \\
\hline Page 11: [1118] Formatted & KPachonska & 3/17/20 2:15:00 PM \\
\hline \multicolumn{3}{|c|}{ Font: 12 pt, Font color: Black } \\
\hline Page 11: [1119] Formatted & KPachonska & 3/17/20 2:15:00 PM \\
\hline \multicolumn{3}{|c|}{ Font: 12 pt, Font color: Black } \\
\hline Page 11: [1120] Formatted & KPachonska & 3/17/20 2:15:00 PM \\
\hline \multicolumn{3}{|c|}{ Font: 12 pt, Font color: Black } \\
\hline Page 11: [1121] Formatted & KPachonska & 3/17/20 2:15:00 PM \\
\hline \multicolumn{3}{|c|}{ Font: 12 pt, Font color: Black } \\
\hline Page 11: [1122] Formatted & KPachonska & 3/17/20 2:15:00 PM \\
\hline
\end{tabular}

Normal, Space After: 8 pt, Line spacing: Multiple 1.15 li, Outline numbered + Level: $1+$ Numbering Style: $1,2,3, \ldots+$ Start at: $1+$ Alignment: Left + Aligned at: $0.25^{\prime \prime}+$ Indent at: $0.5^{\prime \prime}$, Adjust space between Asian text and numbers, Font Alignment: Au

\begin{tabular}{|c|c|c|}
\hline Page 11: [1123] Formatted & KPachonska & 3/17/20 2:15:00 PM \\
\hline \multicolumn{3}{|c|}{ Font: 12 pt, Font color: Black } \\
\hline Page 11: [1124] Formatted & KPachonska & 3/17/20 2:15:00 PM \\
\hline \multicolumn{3}{|c|}{ Font: 12 pt, Font color: Black } \\
\hline Page 11: [1125] Formatted & KPachonska & 3/17/20 2:15:00 PM \\
\hline \multicolumn{3}{|c|}{ Font: 12 pt, Font color: Black } \\
\hline Page 11: [1126] Formatted & KPachonska & 3/17/20 2:15:00 PM \\
\hline \multicolumn{3}{|c|}{ Font: 12 pt, Font color: Black } \\
\hline Page 11: [1127] Formatted & KPachonska & 3/17/20 2:15:00 PM \\
\hline \multicolumn{3}{|c|}{ Font: 12 pt, Font color: Black } \\
\hline Page 11: [1128] Formatted & KPachonska & 3/17/20 2:15:00 PM \\
\hline \multicolumn{3}{|c|}{ Font: 12 pt, Font color: Black } \\
\hline Page 11: [1129] Formatted & KPachonska & 3/17/20 2:15:00 PM \\
\hline \multicolumn{3}{|c|}{ Font: 12 pt, Font color: Black } \\
\hline Page 11: [1130] Formatted & KPachonska & 3/17/20 2:15:00 PM \\
\hline \multicolumn{3}{|c|}{ Font: 12 pt, Font color: Black } \\
\hline Page 11: [1131] Formatted & KPachonska & 3/17/20 2:15:00 PM \\
\hline
\end{tabular}

Font: 12 pt, Font color: Black 


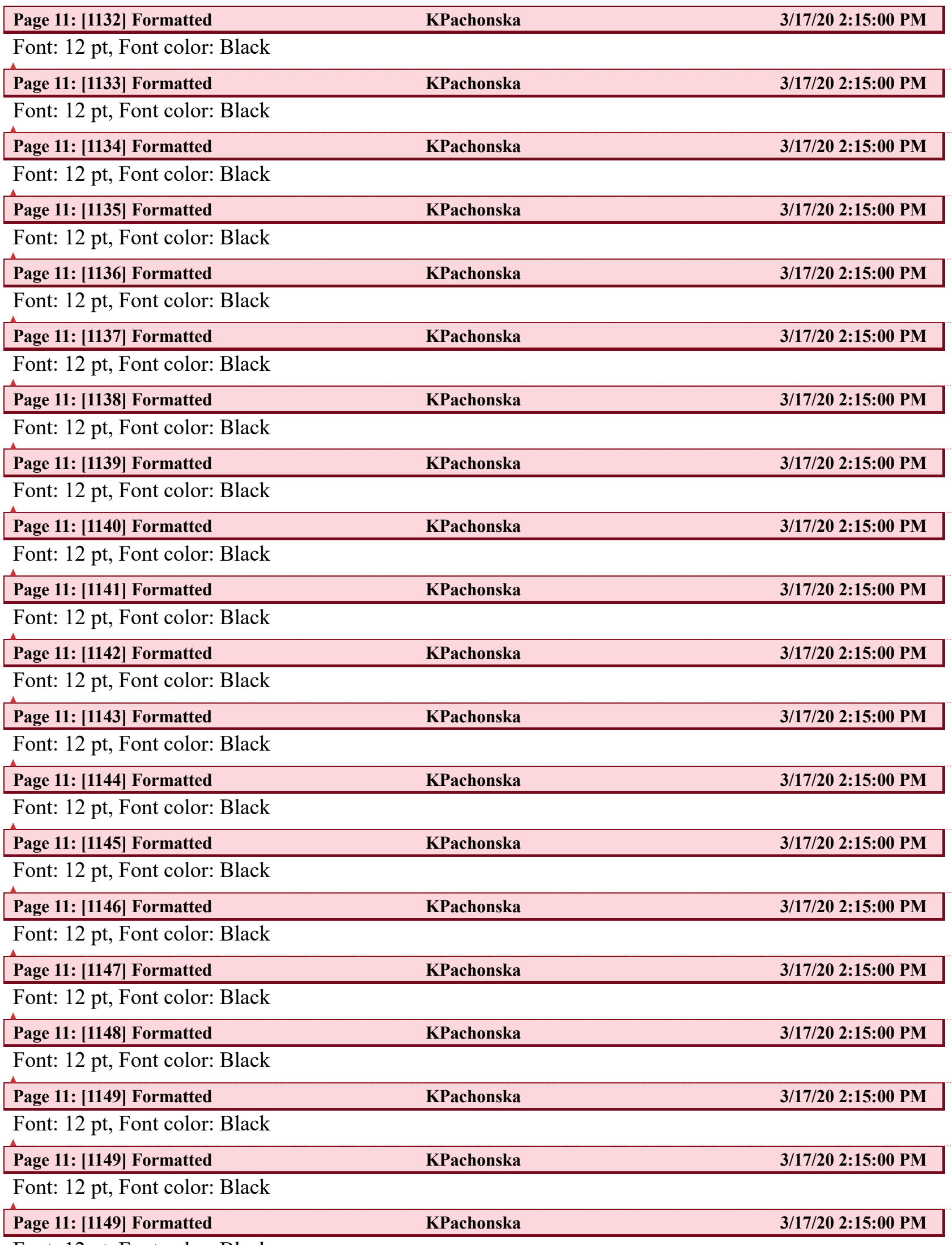

Font: 12 pt, Font color: Black 


\begin{tabular}{|c|c|c|}
\hline Page 1: $[1150]$ Formatted & KPachonska & 3/17/20 2:15:00 PM \\
\hline \multicolumn{3}{|c|}{ Normal, Tab stops: $6.26 "$, Right } \\
\hline Page 1: [1151] Formatted & KPachonska & 3/17/20 2:15:00 PM \\
\hline \multicolumn{3}{|c|}{ Normal, Tab stops: 6.26", Right } \\
\hline Page 12: [1152] Deleted & KPachonska & 3/17/20 2:15:00 PM \\
\hline \multicolumn{3}{|l|}{$x$} \\
\hline \multicolumn{3}{|c|}{ Font: 12 pt, Font color: Black } \\
\hline Page 12: [1154] Formatted & KPachonska & 3/17/20 2:15:00 PM \\
\hline \multicolumn{3}{|c|}{$\begin{array}{l}\text { Normal, Space After: } 8 \text { pt, Line spacing: Multiple } 1.15 \text { li, Outline numbered + Level: } 1+\text { Numbering } \\
\text { Style: } 1,2,3, \ldots+\text { Start at: } 1+\text { Alignment: Left }+ \text { Aligned at: } 0.25 "+\text { Indent at: } 0.5^{\prime \prime} \text {, Adjust space } \\
\text { between Asian text and numbers, Font Alignment: Au }\end{array}$} \\
\hline Page 12: [1155] Formatted & KPachonska & 3/17/20 2:15:00 PM \\
\hline \multicolumn{3}{|c|}{ Default Paragraph Font, Font color: Black } \\
\hline Page 12: [1156] Formatted & KPachonska & 3/17/20 2:15:00 PM \\
\hline \multicolumn{3}{|c|}{ Default Paragraph Font, Font color: Black } \\
\hline Page 12: [1157] Formatted & KPachonska & 3/17/20 2:15:00 PM \\
\hline \multicolumn{3}{|c|}{ Default Paragraph Font, Font color: Black } \\
\hline Page 12: [1158] Formatted & KPachonska & 3/17/20 2:15:00 PM \\
\hline \multicolumn{3}{|c|}{ Default Paragraph Font, Font color: Black } \\
\hline Page 12: [1159] Formatted & KPachonska & 3/17/20 2:15:00 PM \\
\hline \multicolumn{3}{|c|}{ Default Paragraph Font, Font color: Black } \\
\hline Page 12: [1160] Formatted & KPachonska & 3/17/20 2:15:00 PM \\
\hline \multicolumn{3}{|c|}{ Default Paragraph Font, Font color: Black } \\
\hline Page 12: [1161] Formatted & KPachonska & 3/17/20 2:15:00 PM \\
\hline \multicolumn{3}{|c|}{ Default Paragraph Font, Font color: Black } \\
\hline Page 12: [1162] Formatted & KPachonska & 3/17/20 2:15:00 PM \\
\hline \multicolumn{3}{|c|}{ Default Paragraph Font, Font color: Black } \\
\hline Page 12: [1163] Formatted & KPachonska & 3/17/20 2:15:00 PM \\
\hline \multicolumn{3}{|c|}{ Default Paragraph Font, Font color: Black } \\
\hline Page 12: [1164] Formatted & KPachonska & 3/17/20 2:15:00 PM \\
\hline \multicolumn{3}{|c|}{ Default Paragraph Font, Font color: Black } \\
\hline Page 12: [1165] Formatted & KPachonska & 3/17/20 2:15:00 PM \\
\hline \multicolumn{3}{|c|}{ Default Paragraph Font, Font color: Black } \\
\hline Page 12: [1166] Formatted & KPachonska & 3/17/20 2:15:00 PM \\
\hline \multicolumn{3}{|c|}{ Font: 12 pt, Font color: Black } \\
\hline Page 12: [1167] Formatted & KPachonska & 3/17/20 2:15:00 PM \\
\hline \multicolumn{3}{|c|}{ Default Paragraph Font, Font: Font color: Black } \\
\hline Page 12: [1168] Formatted & KPachonska & 3/17/20 2:15:00 PM \\
\hline \multicolumn{3}{|c|}{ Default Paragraph Font, Font color: Black } \\
\hline Page 12: [1168] Formatted & KPachonska & 3/17/20 2:15:00 PM \\
\hline
\end{tabular}


Default Paragraph Font, Font color: Black

Page 12: [1168] Formatted

KPachonska

3/17/20 2:15:00 PM

Page 12: [1168] Formatted

KPachonska

3/17/20 2:15:00 PM

Default Paragraph Font, Font color: Black

\begin{tabular}{|l}
\hline Page 12: [1168] Formatted \\
\hline Default Paragraph Font, Font color: Black
\end{tabular}

Page 12: [1168] Formatted

KPachonska

3/17/20 2:15:00 PM

Default Paragraph Font, Font color: Black

Page 12: [1169] Formatted

KPachonska

3/17/20 2:15:00 PM

Default Paragraph Font, Font color: Black

Page 12: [1169] Formatted

KPachonska

3/17/20 2:15:00 PM

Default Paragraph Font, Font color: Black

Page 12: [1170] Formatted KPachonska

KPachonska

3/17/20 2:15:00 PM

3/17/20 2:15:00 PM

Default Paragraph Font, Font: Not Italic, Font color: Black

Page 12: [1171] Formatted KPachonska

3/17/20 2:15:00 PM

Default Paragraph Font, Font: Not Italic, Font color: Black

Page 12: [1171] Formatted KPachonska

3/17/20 2:15:00 PM

Default Paragraph Font, Font: Not Italic, Font color: Black

\begin{tabular}{|c|c|c|}
\hline Page 12: [1172] Formatted & KPachonska & 3/17/20 2:15:00 PM \\
\hline \multicolumn{3}{|c|}{ Default Paragraph Font, Font color: Black } \\
\hline Page 12: [1172] Formatted & KPachonska & 3/17/20 2:15:00 PM \\
\hline \multicolumn{3}{|c|}{ Default Paragraph Font, Font color: Black } \\
\hline Page 12: [1172] Formatted & KPachonska & 3/17/20 2:15:00 PM \\
\hline \multicolumn{3}{|c|}{ Default Paragraph Font, Font color: Black } \\
\hline Page 12: [1173] Formatted & KPachonska & 3/17/20 2:15:00 PM \\
\hline \multicolumn{3}{|c|}{ Default Paragraph Font, Font color: Black } \\
\hline Page 12: [1174] Formatted & KPachonska & 3/17/20 2:15:00 PM \\
\hline
\end{tabular}

Normal, Line spacing: Multiple 1.15 li, Outline numbered + Level: $1+$ Numbering Style: $1,2,3, \ldots+$

Start at: 1 + Alignment: Left + Aligned at: $0.25 "$ + Indent at: $0.5 "$, Adjust space between Asian text and numbers, Font Alignment: Auto, Pattern: Clear (

\begin{tabular}{|c|c|c|}
\hline Page 12: [1175] Formatted & KPachonska & 3/17/20 2:15:00 PM \\
\hline \multicolumn{3}{|c|}{ Default Paragraph Font, Font color: Black } \\
\hline Page 12: [1176] Formatted & KPachonska & 3/17/20 2:15:00 PM \\
\hline \multicolumn{3}{|c|}{ Default Paragraph Font, Font: Not Italic, Font color: Black } \\
\hline Page 12: [1177] Formatted & KPachonska & 3/17/20 2:15:00 PM \\
\hline \multicolumn{3}{|c|}{ Default Paragraph Font, Font: Not Italic, Font color: Black } \\
\hline Page 12: [1178] Formatted & KPachonska & 3/17/20 2:15:00 PM \\
\hline \multicolumn{3}{|c|}{ Default Paragraph Font, Font: Not Italic, Font color: Black } \\
\hline Page 12: [1179] Formatted & KPachonska & 3/17/20 2:15:00 PM \\
\hline
\end{tabular}


Default Paragraph Font, Font: Not Italic, Font color: Black

Page 12: [1180] Formatted KPachonska

Default Paragraph Font, Font: Not Italic, Font color: Black

Page 12: [1180] Formatted KPachonska 3/17/20 2:15:00 PM

Default Paragraph Font, Font: Not Italic, Font color: Black

Page 12: [1181] Formatted KPachonska

3/17/20 2:15:00 PM

Default Paragraph Font, Font color: Black

Page 12: [1182] Formatted
Default Paragraph Font, Font color: Black

KPachonska

3/17/20 2:15:00 PM

\begin{tabular}{|l}
\hline Page 12: [1182] Formatted \\
Default Paragraph Font, Font color: Black
\end{tabular}

KPachonska

3/17/20 2:15:00 PM

Page 12: [1182] Formatted

KPachonska

3/17/20 2:15:00 PM

Default Paragraph Font, Font color: Black 